\title{
Métodos de Aproximação e Aplicação de MCMC na Estimação de Máxima Verossimilhança para Processos AR(p) e MA(q)
}

\author{
Marcia Fumi Mizoi
}

?ntador: Prof. Dr. Marinho Gomes de Andrade Filho

"Dissertação apresentada ao Instituto de Ciências Matemáticas e de Computação USP, como parte dos requisitos necessários para a obtenção do título de Mestre em Ciências - Area: Ciências de Computação e Matemática Computacional”

São Carlos

-1998- 
"A nobreza do homem está em sentir gratidão pelas

graças recebidas e gravá-las no seu coração."

M. Okada 


\section{Agradecimentos}

Acima de tudo a Deus que através de sua Luz me deu saúde e forças em todos os momentos.

Ao meu orientador prof. Dr. Marinho Gomes de Andrade Filho pela realização deste trabalho, pelo apoio dado principalmente no início e também pela possibilidade de iniciar carreira na área estatística.

Ao João Eduardo pelo apoio, carinho e amor nos momentos mais difíceis.

Aos professores do ICMC que contribuiram para a minha formação, em especial aos professores doutores Cassilda Maria Ribeiro e Marcos Nereu Arenales pelas contribuições dadas no exame de qualificação.

À todos os amigos e à todos os funcionários do campus USP - São Carlos que direta ou indiretamente me ajudaram na realização deste trabalho.

Ao Conselho Nacional de Desenvolvimento Científico e Tecnológico - CNPq pelo apoio financeiro fornecido. 


\section{Resumo}

Neste projeto, abordamos os modelos de séries temporais estacionárias do tipo AR(p) e MA(q). O interesse é obter para estes modelos as estimativas de máxima verossimilhança exata. A diferenciação explícita da função de verossimilhança exata para se obter estas estimativas, não é recomendável por envolver operações complicadas. Assim, [Box, Jenkins e Reinsel - 1994] sugerem métodos numéricos baseados em aproximações. Em [Miller - 1995] são apresentadas expressōes mais simples para as derivadas da função de verossimilhança junto com um algoritmo iterativo, no caso de modelos $\mathrm{AR}(\mathrm{p})$. O objetivo do presente projeto é propor o uso de algoritmos de simulação de Monte Carlo com Cadeia de Markov (MCMC) para o cálculo das estimativas de máxima verossimilhança. Aqui, os algoritmos utilizados foram o amostrador de Gibbs em conjunto com o algoritmo de Metropolis-Hastings. Os resultados obtidos usando MCMC são comparados com as estimativas feitas pelos métodos numéricos propostos em [Box, Jenkins e Reinsel - 1994] e [Miller - 1995]. 


\begin{abstract}
In this work, the autoregressive and moving average time series models are considered. The main objective here is to use Markov Chain Monte Carlo (MCMC) method (Metropolis-Hastings algorithm and the Gibbs Sampler) to calculate the maximum likelihood estimates in the ordinary sense (a point in the parameter space that maximizes the likelihood). The porpoused method was applied to the simulated and real date record. The estimates obtained by MCMC method was compared with likelihood estimate by iterative numerical methods.
\end{abstract}




\section{ÍNDICE}

Capítulo 1 - Séries Temporais

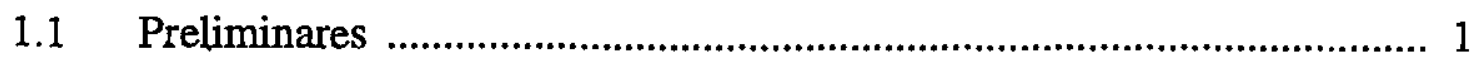

1.2 Objetivos da Análise de Séries Temporais .......................................... 4

1.3 Modelos para Séries Temporais ....................................................... 5

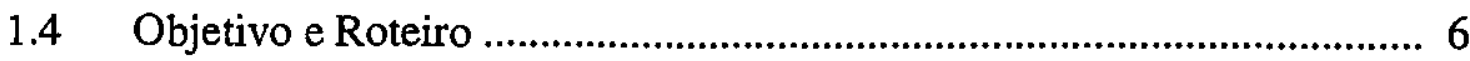

Capítulo 2 - Modelos Lineares Estacionários $\quad 8$

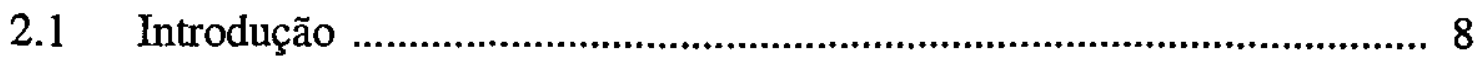

2.2 Condições de Estacionariedade e Invertibilidade ................................... 10

2.3 Modelos Auto-regressivos de Ordem p - AR(p) .................................. 11

2.3.1 Condições de Estacionariedade e Invertibilidade ....................... 11

2.3.2 Função de Auto-correlação ....................................................... 12

2.4 Modelos de Médias Móveis de Ordem q - MA(q) ................................. 15

2.4.1 Condições de Estacionariedade e Invertibilidade ...................... 15

2.4.2 Função de Auto-correlação ...................................................... 16

2.5 Modelos Mistos Auto-regressivos - Médias Móveis .............................. 18

2.5.1 Condições de Estacionariedade e Invertibilidade ...................... 18

2.5.2 Função de Auto-correlação ..................................................... 18

2.6 Função de Auto-correlação Parcial ...................................................... 19

Capítulo 3 - Função de Verossimilhança Exata

3.1 Função de Verossimilhança Exata para um Processo Auto-regressivo ................................................................. 22

3.2 Função de Verossimilhança Exata para um Processo de Médias-móveis 28 


\section{Capítulo 4 - Métodos Numéricos para Estimativas de Máxima Verossimilhança}

4.1 Métodos Numéricos para Estimativas de Máxima Verossimilhança para Processos AR(p)

4.1.1 Estimativas Mínimos Quadrados ............................................... 33

4.1.2 Aproximação das Estimativas de Máxima Verossimilhança....... 34

4.1.3 ARMLE

4.2 Métodos Numéricos para Estimativas de Máxima Verossimilhança para Processos MA(q) .......................................................................... 40

4.2.1 Estimação de Máxima Verossimilhança Condicional ................. 40

4.2.2 Estimação de Máxima Verossimilhança Incondicional .............. 42

Capítulo 5 - Método de Simulação de Monte Carlo com Cadeia de Markov (MCMC) para Estimativas de Máxima Verossimilhança

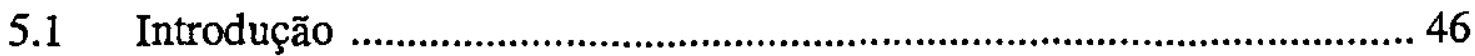

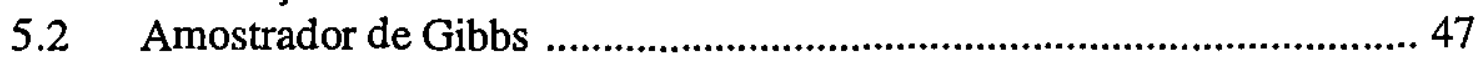

5.3 Algoritmo de Metropolis-Hastings ........................................................ 48

5.4 Critério de Convergência de Gelman e Rubin ........................................ 49

5.5 Aplicação de Métodos MCMC para Processos AR(p) ........................... 51

5.6 Aplicação de Métodos MCMC para Processos MA(q) …........................ 53

Capítulo 6 - Aplicação $\quad 56$

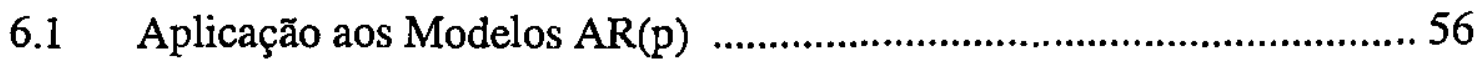

6.2 Aplicação aos Modelos MA(q) ............................................................ 77

Capítulo 7 - Conclusão 91

$\begin{array}{ll}\text { Bibliografia } & 93\end{array}$

$\begin{array}{ll}\text { Apêndice } & 95\end{array}$ 


\section{Capítulo 1 - Séries Temporais}

\subsection{Preliminares}

Uma série temporal é qualquer conjunto de observaçōes seqüenciais no tempo.

Exemplos de séries temporais ocorrem em várias áreas, como economia, engenharia, medicina, ciências sociais, meteorologia e oceanografia.

Representaremos a série temporal por $\mathrm{z}_{t}, t \epsilon \mathrm{T}$, onde $\mathrm{T}$ é um conjunto dos instantes de observações. Quando T for um conjunto finito ou enumerável, como o conjunto dos inteiros, a série temporal é dita discreta e quando $\mathrm{T}$ for um intervalo da reta, a série é dita contínua.

Em nossos estudos, trataremos de séries temporais discretas com observações feitas em intervalos eqüidistantes no tempo onde denotaremos por $z_{i}$ a observação feita no instante $t_{i}$, ou seja, $z_{i}=z_{t_{i}}, i=1,2, \ldots, n$.

A seguir são dados alguns exemplos reais de séries temporais com seus respectivos gráficos.

a) Consumo de energia elétrica no Estado do Espírito Santo de 1977 a 1978, com 141 observações mensais [Morettin e Toloi - 1981];

b) Importações feitas pelo Brasil de 1973 a 1974, com 150 observações mensais ,[Morettin e Toloi - 1981];

c) Índice do custo de vida de São Paulo de 1976 a 1977, com 126 observações mensais [Morettin e Toloi - 1981];

d) Média mensal da temperatura do ar em Recife de 1953 a 1962 [Chatfield - 1989]; 
e) Total mensal de passageiros da linha aérea internacional, em milhares, de 1949 a 1960 [Chatfield - 1989].

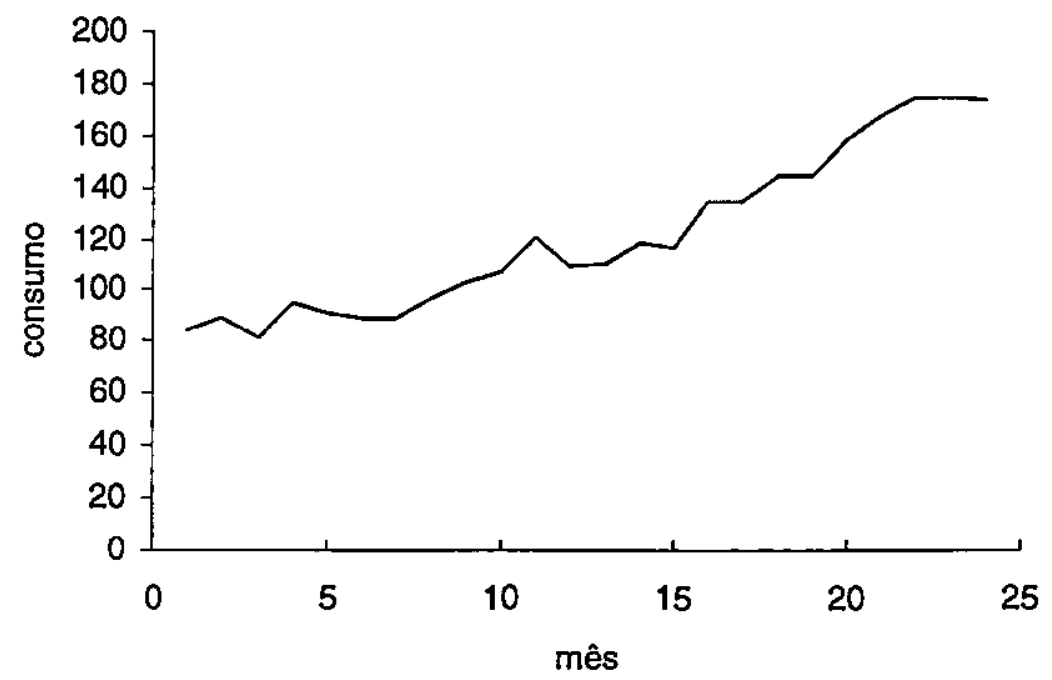

fig.1 - Consumo de energia elétrica no Espírito Santo (Mwh) - janeiro de 1977 a dezembro de 1978.

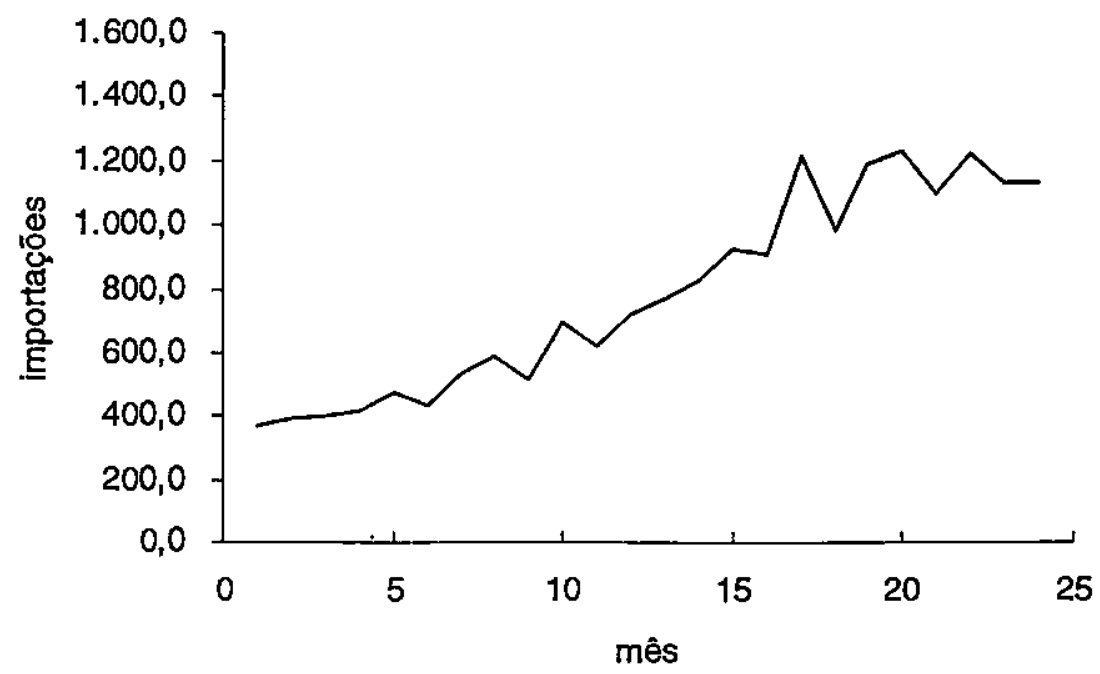

fig. 2 - Importações feitas pelo Brasil (10 US\$) - janeiro de 1973 a dezembro de 1974. 


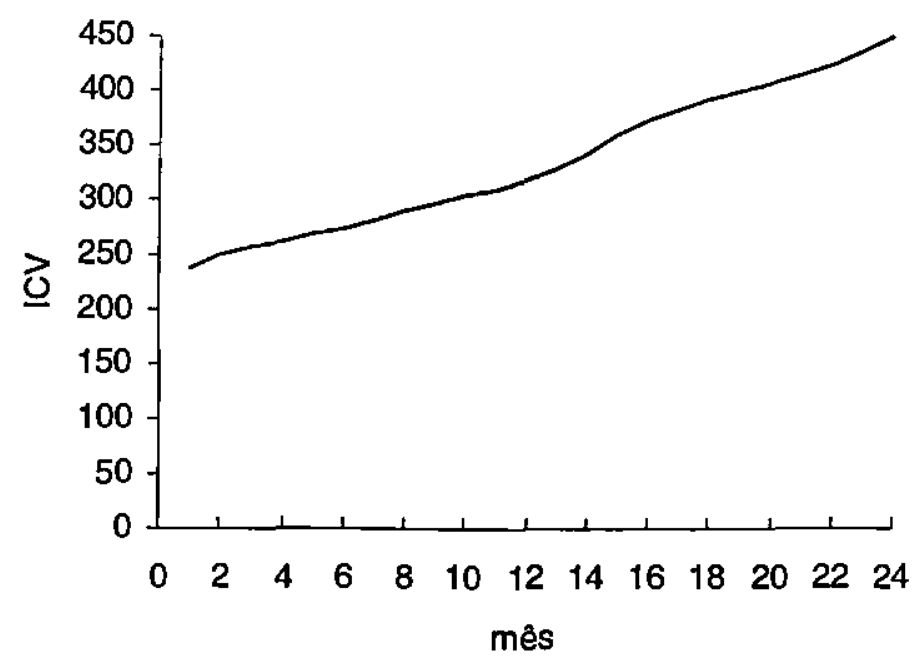

fig.3 - Índice de Custo de Vida de São Paulo - janeiro de 1976 a dezembro 1977.

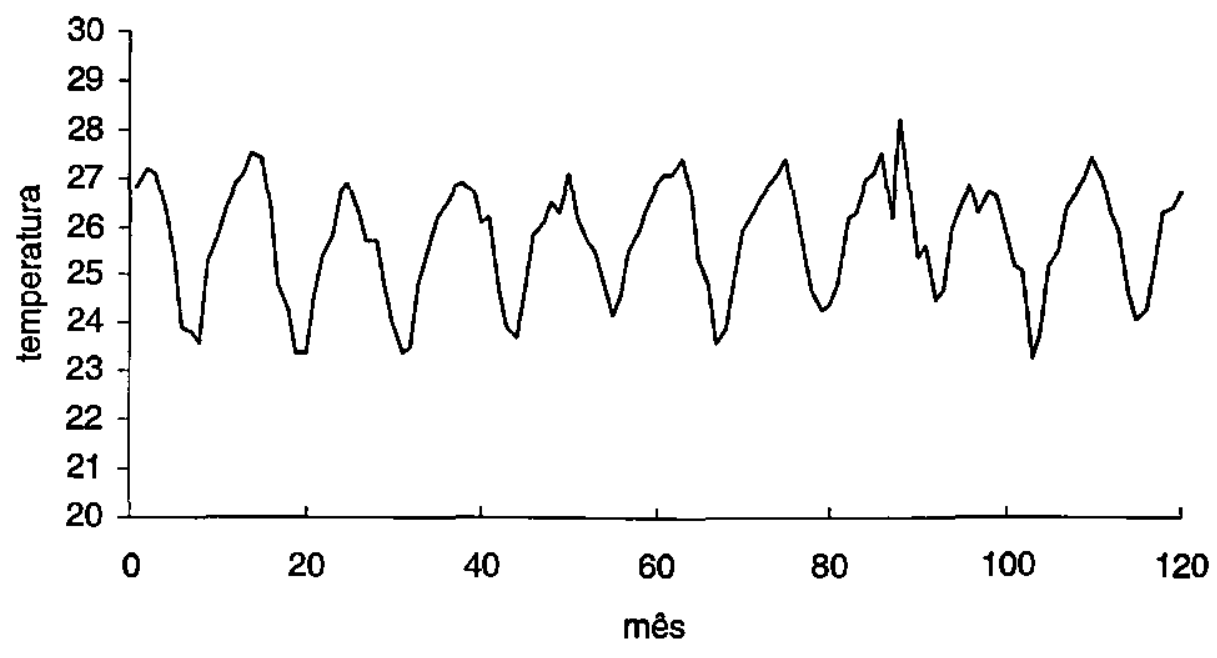

fig.4 - Média mensal da temperatura do ar $\left({ }^{\circ} \mathrm{C}\right)$ em Recife - janeiro de 1953 a dezembro de 1962. 


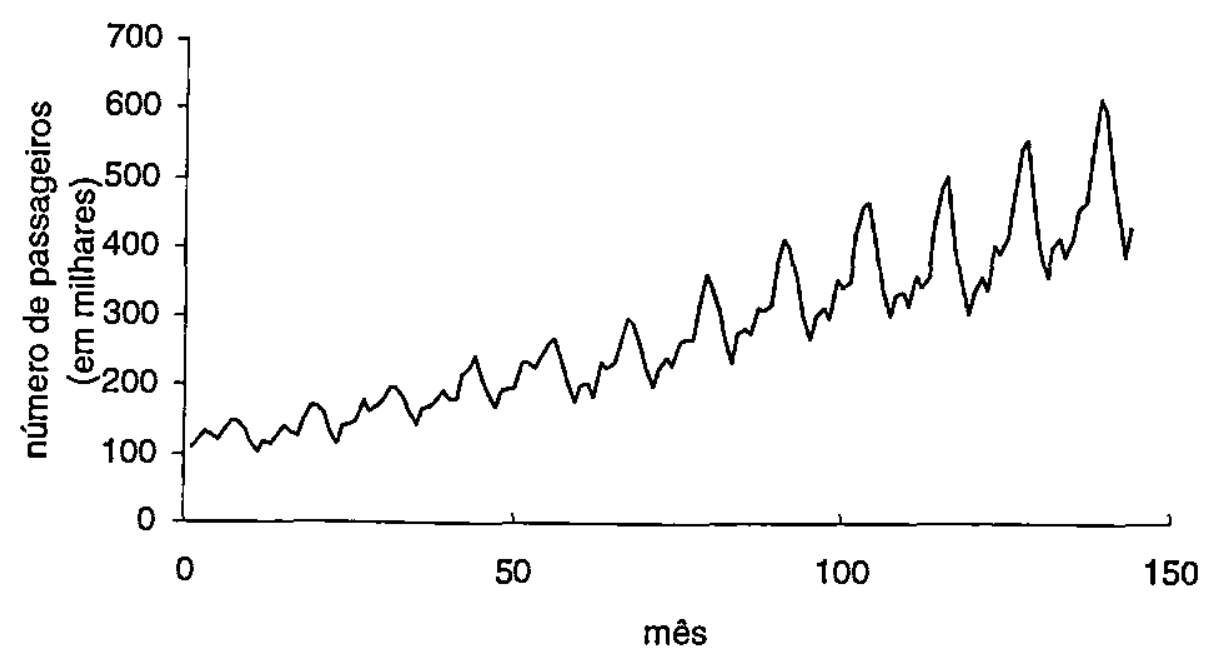

fig.5 Total mensal de passageiros da linha aérea internacional - janeiro de 1949 a dezembro de 1960.

\subsection{Objetivos da Análise de Séries Temporais}

Os objetivos principais da análise de séries temporais são:

a) investigar o mecanismo gerador da série temporal;

b) descrever o comportamento da série;

c) fazer previsões de valores futuros da série;

d) procurar periodicidades relevantes nos dados.

Através da análise da série temporal construimos modelos matemáticos para a série. Esta análise pode ser feita no domínio temporal, onde os modelos propostos são modelos paramétricos, ou no domínio de freqüências, onde os modelos propostos são nãoparamétricos. 


\subsection{Modelos para Séries Temporais}

Os modelos utilizados para descrever séries temporais são processos estocásticos, isto é, processos controlados por leis probabilísticas.

Um processo estocástico é uma família de variáveis aleatórias $\left\{\mathrm{z}_{t}(w), t \in \mathrm{T}, w \in \Omega\right\}$ definidas num espaço de probabilidades $(\Omega, \mathrm{A}, \mathrm{P})$, tal que, para cada $t \epsilon \mathrm{T}, \mathrm{z}_{\imath}(w)$ é uma variável aleatória e para cada $w \epsilon \Omega$ fixado, $\mathrm{z}_{t}$ é uma função de $t$, ou seja, uma realização ou trajetória do processo. Cada uma dessas trajetórias representa uma série temporal.

Uma suposição que normalmente é feita no estudo de modelos para séries temporais, é a de que o processo é estacionário, ou seja, o processo se desenvolve no tempo de modo que a origem não é importante.

Formalmente há dois tipos de estacionariedade: forte (ou estrita) e fraca (ou ampla, ou de segunda ordem).

Definição 1: Um processo estocástico $\left\{z_{t}(w), t \in \mathrm{T}, w \in \Omega\right\}$ é fortemente estacionário se suas distribuições finito-dimensionais são invariantes sob translações no tempo, isto é,

$$
\mathrm{F}\left(\mathrm{z}_{1}, \ldots, \mathrm{z}_{\mathrm{n}} ; t_{1}+\tau, \ldots, t_{n}+\tau\right)=\mathrm{F}\left(\mathrm{z}_{1}, \ldots, \mathrm{z}_{\mathrm{n}} ; t_{1}, \ldots, t_{\mathrm{n}}\right)
$$

para quaisquer $t_{1}, \ldots, t_{n}, \tau \in \mathrm{T}$.

Definição 2: Um processo estocástico $\left\{\mathrm{z}_{t}(w), t \in \mathrm{T}, w \in \Omega\right\}$ é fracamente estacionário se, e somente se:

i) $E\left[\mathrm{z}_{t}\right]=\mathrm{m}$, constante, para qualquer $t \epsilon \mathrm{T}$;

ii) $\operatorname{Var}\left[\mathrm{z}_{i}\right]=\sigma^{2}$, constante, para qualquer $t \epsilon \mathrm{T}$;

iii) $\operatorname{Cov}\left[\mathrm{z}_{t_{1}}, \mathrm{z}_{t_{2}}\right]=\gamma_{t_{2}-t_{1}}$ é uma função de $t_{2}-t_{1}$. 
Processos fracamente estacionários cujas distribuiçōes finito-dimensionais são gaussianos são automaticamente fortemente estacionários. Estaremos interessados aqui somente nos processos gaussianos fracamente estacionários, os quais serão denominados a partir de agora simplesmente processos estacionários. No caso de processos não estacionários homogêneos, isto é, processos cujo nível e/ou inclinação mudam com o decorrer do tempo, estes podem ser transformados por diferenças sucessivas da série original, ou usando outros tipos de transformações [Box e Cox - 1964], até se obter a estacionariedade.

A classe de modelos para séries temporais que vamos abordar aqui são os modelos paramétricos auto-regressivos $\mathrm{AR}(\mathrm{p})$ e de médias-móveis $\mathrm{MA}(\mathrm{q})$ que serão introduzidos no próximo capítulo.

\subsection{Objetivo e Roteiro}

$\mathrm{Na}$ análise de séries temporais, um dos passos importantes é a estimação dos parâmetros do modelo matemático ajustado à série dada. Uma maneira de proceder esta estimação é através do uso de métodos de estimativas de máxima verossimilhança. No nosso caso estamos interessados em obter as estimativas para os modelos de séries temporais estacionárias do tipo $\mathrm{AR}(\mathrm{p})$ e $\mathrm{MA}(\mathrm{q})$. A complexidade da expressão da função de verossimilhança exata para estes modelos sugere o uso de métodos numéricos baseados em aproximações como os apresentados em [Box, Jenkins e Reinsel - 1994]. Também em [Miller - 1995] é proposto um método numérico, para o caso de modelos AR(p), que utiliza um algoritmo iterativo baseado em expressōes mais simples para as derivadas da função de verossimilhança. Um método proposto para estimar os parâmetros de modelos MA(1) é apresentado em [Anderson, et al - 1996]. O objetivo deste projeto é propor como método de obtenção das estimativas de máxima verossimilhança, o uso de algoritmos de simulação de 
Monte Carlo com Cadeia de Markov (MCMC), mais especificamente o algoritmo amostrador de Gibbs [Casella e George - 1992] e o algoritmo de Metropolis-Hastings [Chib e Greenberg 1995]. O uso de simulação de Monte Carlo para cálculo de estimativas de máxima verossimilhança foi proposto inicialmente por [Geyer - 1992] e [Geyer - 1994]. Nesse trabalho é usado programação linear para encontrar o suporte da função de verossimilhança e então acha ${ }^{N}$ a distribuição da família exponencial neste suporte. Na proposta do nosso trabalho os parâmetros dos modelos são interpretados como variáveis aleatórias cuja densidade tem como núcleo a função de verossimilhança do modelo, então os algoritmos amostrador de Gibbs e Metropolis-Hastings são usados para gerar uma amostra desses parâmetros e a estimativa de máxima verossimilhança desses parâmetros é tomada como sendo a moda da amostra gerada. Comparações entre os resultados da aplicação dos métodos numéricos e da aplicação de MCMC foram realizadas para algumas séries temporais.

No capítulo 2 introduzimos os conceitos fundamentais da metodologia de Box \& Jenkins para modelos de séries temporais estacionárias e descrevemos as propriedades básicas ṇecessárias para a identificação do tipo de modelo. A formulação da expressão da função de verossimilhança exata para os modelos $\mathrm{AR}(\mathrm{p})$ e $\mathrm{MA}(\mathrm{q})$ é detalhada no capítulo 3 e a seguir, no capítulo 4, apresentamos os métodos numéricos propostos em [Box, Jenkins e Reinsel 1994] e em [Miller - 1995] para a obtenção das estimativas de máxima verossimilhança para cada um destes modelos. Iniciamos o capítulo 5 apresentando os algoritmos de simulação MCMC, amostrador de Gibbs e Metropolis-Hastings. Em seguida, descrevemos o desenvolvimento da teoria necessária para a aplicação destes algoritmos nos modelos $\mathrm{AR}(\mathrm{p}) \mathrm{e}$ MA(q). No capítulo 6 discutimos a aplicação dos métodos numéricos e do algoritmo MCMC no caso específico de séries geradas por modelos $\operatorname{AR}(1), \operatorname{AR}(2), M A(1)$ e $M A(2)$ fazendo comparações entre os resultados obtidos. Finalmente, no capítulo 7 discutimos as conclusões e as perspectivas de extensão do presente trabalho. 


\section{Capítulo 2 - Modelos Lineares Estacionários}

\subsection{Introdução}

Os modelos que analisaremos aqui são casos particulares de um modelo de filtro linear. Neste modelo uma série de entrada $a_{t}$ é convertida em outra série $y_{t}$ através de uma função de transferência, onde a série $a_{t}$ é um ruído branco. Um ruído branco consiste de uma seqüência de variáveis aleatórias não correlacionadas, com média zero e variância constante $\sigma_{a}^{2}$

Formalmente, temos

$$
\begin{aligned}
y_{t} & =\mu+a_{t}+\psi_{1} a_{t-1}+\psi_{2} a_{t-2}+\ldots \\
& =\mu+a_{t}+\sum_{j=1}^{\infty} \psi_{j} a_{t-j}
\end{aligned}
$$

onde $\mu$ é um parâmetro que determina o nível série. Quando $\sum_{j=1}^{\infty} \psi_{j}$ converge, então $\mu=E\left[y_{t}\right]$.

Chamando $\mathrm{z}_{t}=y_{t}-\mu$, temos

$$
y_{t}=\mu \omega_{t}+\underset{\substack{\infty \\ c_{i}}}{1} \psi_{j} a_{t-j}
$$

$$
\begin{aligned}
& \mathrm{z}_{t}=a_{t}+\sum_{j=1}^{\infty} \psi_{j} a_{t-j} \\
& y t-\mu=a_{t}+\sum_{j=1}^{\infty} \psi_{j} u_{t-j}
\end{aligned}
$$

Também podemos escrever $\mathrm{z}_{t}$, alternativamente, como uma somá ponderada de valores passados $\mathrm{z}_{t-1}, \mathrm{z}_{t-2}, \ldots$, mais um ruído $a_{t}$, isto é,

$$
\begin{aligned}
\mathrm{z}_{t} & =\pi_{1} \mathrm{z}_{t-1}+\pi_{2} \mathrm{z}_{t-2}+\ldots+a_{t} \\
& =\sum_{j=1}^{\infty} \pi_{j} \mathrm{z}_{t-j}+a_{t}
\end{aligned}
$$


As relações entre os pesos $\psi$ e os pesos $\pi$ podem ser obtidas usando o operador translação para o passado, denotado por B e definido por:

$$
\mathrm{Bz}_{t}=\mathrm{z}_{t-1}
$$

e

$$
\mathrm{B}^{m} \mathrm{z}_{t}=\mathrm{z}_{t-m}
$$

A expressão (2.2) pode então ser escrita como

$$
\mathrm{z}_{t}=\left(1+\sum_{j=1}^{\infty} \psi_{j} \mathrm{~B}^{j}\right) a_{t}
$$

ou

$$
\mathrm{z}_{t}=\psi(\mathrm{B}) a_{\mathrm{t}}
$$

onde

$$
\psi(\mathrm{B})=\sum_{j=0}^{\infty} \psi_{j} \mathrm{~B}^{\mathrm{j}}
$$

$\operatorname{com} \psi_{0}=1$

O operador $\psi(\mathrm{B})$ acima, é o operador linear que transforma $a_{t}$ em $\mathrm{z}_{t}$ e é chamado função de transferência do filtro linear.

Analogamente, a expressão (2.3) pode ser escrita como

$$
\left(1-\sum_{j=1}^{\infty} \pi_{j} \mathrm{~B}^{\mathrm{j}}\right) \mathrm{z}_{t}=a_{t}
$$

ou

$$
\pi(\mathrm{B}) \mathrm{z}_{t}=a_{t}
$$


onde $\pi(\mathrm{B})$ é o operador

$$
\pi(\mathrm{B})=1-\sum_{j=1}^{\infty} \pi_{j} \mathrm{~B}^{\mathrm{j}}
$$

De (2.4) e (2.5), segue que:

$$
\pi(\mathrm{B}) \psi(\mathrm{B}) a_{t}=a_{t}
$$

e, portanto

$$
\pi(\mathrm{B})=\psi^{-1}(\mathrm{~B})
$$

Com esta relação, podemos obter os pesos $\pi_{j}$, conhecendo os pesos $\psi_{j}$, e vice-versa.

\subsection{Condições de Estacionariedade e Invertibilidade}

Para um processo linear, pode ser demonstrado que a estacionariedade é garantida se as raízes do polinômio $\psi(\mathrm{B})$ estiverem dentro ou sobre o círculo unitário e, para que o processo seja invertível, a condição é que a série $\pi(\mathrm{B})$ convirja dentro ou sobre o círculo unitário. Então, temos a seguinte proposição:

Proposição 1: Um processo linear será estacionário se a série $\psi(\mathrm{B})$ convergir para $|\mathrm{B}| \leq 1$ e será invertível se $\pi(\mathrm{B})$ convergir para $|\mathrm{B}| \leq 1$. 


\subsection{Modelos Auto-regressivos de Ordem $\mathbf{p}-\operatorname{AR}(\mathbf{p})$}

Consideremos a expressão (2.3) no caso especial em que somente um número finito de pesos $\pi_{j}$ é diferente de zero, ou seja, $\pi_{1}=\phi_{1}, \pi_{2}=\phi_{2}, \ldots, \pi_{\mathrm{p}}=\phi_{\mathrm{p}}$ e $\pi_{\mathrm{k}}=0$ para $\mathrm{k}>\mathrm{p}$. Obtemos então, um modelo auto-regressivo de ordem p, que denotaremos de agofa em diante como:

$$
\mathrm{z}_{\mathrm{t}}=\phi_{1} \mathrm{z}_{t-1}+\phi_{2} \mathrm{z}_{\mathrm{t}-2}+\ldots+\phi_{p} \mathrm{z}_{t-p}+a_{t}
$$

O modelo acima é usualmente identificado por $\mathrm{AR}(\mathrm{p})$.

Utilizando o operador B definido anteriormente, também podemos escréver (2.7) na forma

$$
\phi(\mathrm{B}) \mathrm{z}_{t}=a_{t}
$$

onde

$$
\phi(\mathrm{B})=1-\phi_{1} \mathrm{~B}-\phi_{2} \mathrm{~B}^{2}-\ldots-\phi_{p} \mathrm{~B}^{p}
$$

é o operador auto-regressivo de ordem p.

Proposição 2: Um processo $\mathrm{AR}(\mathrm{p})$ é estacionário se as raízes do polinômio $\phi(\mathrm{B})=0$ estão todas fora do círculo de raio unitário.

A proposição 2 é uma conseqüência do conceito de estacionariedade dado na proposiçãol como veremos a seguir.

\subsubsection{Condições de Estacionariedade e Invertibilidade}

O conjunto de parâmetros $\phi_{1}, \phi_{2}, \ldots, \phi_{p}$ de um processo AR(p) deve satiṣfazer certas condições para que o processo seja estacionário. 
Sejam $\mathrm{G}_{i}^{-1}, i=1, \ldots, \mathrm{p}$, as raízes do polinômio $\phi(\mathrm{B})=0$. Então,

$$
\phi(B)=\left(1-G_{1} B\right)\left(1-G_{2} B\right) \ldots\left(1-G_{p} B\right)
$$

e, expandindo em frações parciais

$$
\phi^{-1}(\mathrm{~B})=\frac{1}{\prod_{i=1}^{p}\left(1-\mathrm{G}_{i} \mathrm{~B}\right)}=\sum_{i=1}^{p} \frac{\mathrm{A}_{i}}{\left(1-\mathrm{G}_{i} \mathrm{~B}\right)}
$$

onde $\mathrm{A}_{i}, i=1, \ldots, \mathrm{p}$, são constantes. Então:

$$
\mathrm{z}_{t}=\phi^{-1}(\mathrm{~B}) a_{\mathrm{t}}=\sum_{i=1}^{p} \frac{\mathrm{A}_{i}}{\left(1-\mathrm{G}_{i} \mathrm{~B}\right)} a_{\mathrm{t}}
$$

Para termos a estacionariedade, $\psi(\mathrm{B})=\phi^{-1}(\mathrm{~B})$ deve convergir para $|\mathrm{B}|\lfloor 1$, ou seja, devemos ter $\left|G_{i}\right|<1, i=1,2, \ldots$, p. Esta condição é equivalente à de que as raízes da ẹquação $\phi(B)=0$ estejam fora do círculo unitário.

Visto que a série $\pi(\mathrm{B})=\phi(\mathrm{B})=1-\phi_{1} \mathrm{~B}-\phi_{2} \mathrm{~B}^{2}-\ldots-\phi_{\mathrm{p}} \mathrm{B}^{p}$ é finịta, não há restrições sobre os parâmetros de um processo auto-regressivo para assegurar a invertibilidade.

\subsubsection{Função de Auto-correlação}

Multiplicando ambos os membros da equação (2.7) por $\mathrm{z}_{t-j}$, temos

$$
\mathrm{z}_{t-j} \mathrm{z}_{t}=\phi_{1} \mathrm{z}_{t-j} \mathrm{z}_{t-1}+\ldots+\phi_{p} \mathrm{z}_{t-j} \mathrm{z}_{t-p}+\mathrm{z}_{t-j} a_{t}
$$


e, denotando por $\gamma_{j}=E\left[z_{t-j} z_{t}\right], \gamma_{b-i t}=E\left[z_{t-j} z_{t-i}\right]$, temos:

$$
\gamma_{j}=\phi_{1} \gamma_{j-1}+\phi_{2} \gamma_{j-2}+\ldots+\phi_{p} \gamma_{j-p} \quad j>0
$$

pois $E\left[a_{t} \mathrm{z}_{t-j}\right]=0, j>0$

Dividindo por $\gamma_{0}$, vem

$$
\rho_{j}=\phi_{1} \rho_{j-1}+\phi_{2} \rho_{j-2}+\ldots+\phi_{p} \rho_{j-p} \quad j>0
$$

Vemos da expressão acima que a função de auto-correlação $\rho_{f}$ é determinada pela equação:

$$
\phi(\mathrm{B}) \rho_{j}=0
$$

para $j>0$.

Então, escrevendo

$$
\phi(B)=\prod_{i=1}^{p}\left(1-G_{i} B\right)
$$

pode ser mostrado que a solução geral de (2.8) é

$$
\rho_{j}=A_{1} G_{1}^{j}+A_{2} G_{2}^{j}+\ldots+A_{p} G_{p}^{j}
$$

Portanto, a função de auto-correlação de um processo auto-regressivo decai de acordo com exponenciais e/ou senóides amortecidas. 
Se substituirmos $j=1,2, \ldots, \mathrm{p}$ em (2.8), obteremos um conjunto de equações lineares da forma

$$
\begin{aligned}
\rho_{1} & =\phi_{1} \quad+\phi_{2} \rho_{1}+\ldots+\phi_{p} \rho_{p-1} \\
\rho_{2} & =\phi_{1} \rho_{1}+\phi_{2}+\ldots+\phi_{p} \rho_{p-2} \\
& \vdots \\
\rho_{p} & =\phi_{1} \rho_{p-1}+\phi_{2} \rho_{p-2}+\ldots+\phi_{p}
\end{aligned}
$$

que são chamadas equações de Yule-Walker.

$\mathrm{Na}$ forma matricial, escrevemos

$$
\left[\begin{array}{cccc}
1 & \rho_{1} & \ldots & \rho_{p-1} \\
\rho_{1} & 1 & \ldots & \rho_{p-2} \\
\cdot & \cdot & \cdot & \cdot \\
\cdot & \cdot & \cdot & \cdot \\
\cdot & \cdot & \cdot & \cdot \\
\rho_{p-1} & \rho_{p-2} & \cdots & 1
\end{array}\right]\left[\begin{array}{c}
\phi_{1} \\
\phi_{2} \\
\cdot \\
\cdot \\
\cdot \\
\phi_{p}
\end{array}\right]=\left[\begin{array}{c}
\rho_{1} \\
\rho_{2} \\
\cdot \\
\cdot \\
\cdot \\
\rho_{p}
\end{array}\right]
$$

As funções de auto-correlações $\rho_{j}$ acima, podem ser estimadas usando as seguintes funções de auto-correlações amostrais $r_{j}$ :

$$
\mathrm{r}_{j}=\frac{\mathrm{c}_{j}}{\mathrm{c}_{0}}
$$

onde

$$
c_{j}=\frac{1}{n} \sum_{t=1}^{n-j}\left(\mathrm{z}_{t}-\overline{\mathrm{z}}\right)\left(\mathrm{z}_{\mathrm{t}+j}-\overline{\mathrm{z}}\right)
$$

é a função de auto-covariância amostral e

$$
\overline{\mathrm{z}}=\frac{1}{n} \sum_{t=1}^{n} \mathrm{z}_{\mathrm{t}}
$$

é a média amostral. Geralmente os valores de $c_{j}$ são calculados para $j \leq 4$. 
Substituindo $\rho_{j}$ por suas estimativas $r_{j}$ em (2.10), podemos obter estimativas dos parâmetros $\phi_{1}, \phi_{2}, \ldots, \phi_{\mathrm{p}}$ do modelo $\mathrm{AR}(\mathrm{p})$. Este método de estimar os parâmetros é chamado método dos momentos e oferece estimativas ruins quando o processo está próximo da fronteira da região de estacionariedade no espaço dos parâmetros.

\subsection{Modelos de Médias Móveis de Ordem q - MA(q)}

$\mathrm{Na}$ expressão (2.2), se tivermos somente um número finito de pesos diferente de zero, isto é, $\psi_{1}=-\theta_{1}, \psi_{2}=-\theta_{2}, \ldots, \psi_{q}=-\theta_{q}$ e $\psi_{\mathrm{k}}=0$ para $\mathrm{k}>\mathrm{q}$, então o processo resultante é dado por:

$$
\mathrm{z}_{t}=a_{t}-\theta_{1} a_{i-1}-\theta_{2} a_{t-2}-\ldots-\theta_{q} a_{t-q}
$$

o qual é chamado processo de médias móveis de ordem q, e denotado por MA(q).

De forma equivalente, podemos escrever

$$
\mathrm{z}_{i}=\theta(\mathrm{B}) a_{i}
$$

onde

$$
\theta(\mathrm{B})=1-\theta_{1} \mathrm{~B}-\theta_{2} \mathrm{~B}^{2}-\ldots-\theta_{q} \mathrm{~B}^{q}
$$

é o operador de médias móveis de ordem q.

\subsubsection{Condições de Estacionariedade e Invertibilidade}

Visto que a série

$$
\psi(\mathrm{B})=\theta(\mathrm{B})=1-\theta_{1} \mathrm{~B}-\theta_{2} \mathrm{~B}^{2}-\ldots-\theta_{q} \mathrm{~B}^{q}
$$


é finita, não há restriçōes sobre os parâmetros para que o processo seja estacionário.

Vamos verificar então, quais as condições para a invertibilidade do processo $M A(q)$

$$
\mathrm{z}_{\mathrm{t}}=\theta(\mathrm{B}) a_{\mathrm{t}}
$$

Temos que

$$
a_{\mathrm{t}}=\theta^{-1}(\mathrm{~B}) \mathrm{z}_{\mathrm{t}}
$$

Portanto, se $\mathrm{H}_{j}^{-1}, j=1, \ldots, \mathrm{q}$, são as raízes do polinômio $\theta(\mathrm{B})=0$, temos:

$$
\theta(\mathrm{B})=\prod_{j=1}^{\mathrm{q}}\left(1-\mathrm{H}_{j} \mathrm{~B}\right)
$$

e então, expandindo em frações parciais,

$$
\pi(\mathrm{B})=\theta^{-1}(\mathrm{~B})=\sum_{j=1}^{\mathrm{q}} \frac{\mathrm{M}_{j}}{\left(1-\mathrm{H}_{j} \mathrm{~B}\right)}
$$

Para que o processo seja invertível, temos que $\pi(B)$ deve convergir para $|B| \leq 1$, ou seja, devemos ter $\left|\mathrm{H}_{j}\right|<1, j=1,2, \ldots, \mathrm{q}$. Visto que $\mathrm{H}_{j}^{-1}$ são as raízes de $\theta(\mathrm{B})=0$, segue que a condição de invertibilidade para um processo $\mathrm{MA}(\mathrm{q})$ é que as raízes do polinômio $\theta(\mathrm{B})=0$ estejam fora do círculo unitário.

\subsubsection{Função de Auto-correlação}

Usando a equação (2.11), obtemos que a função de autocovariância de um processo MA(q)é

$$
\gamma_{j}=E\left[\mathrm{z}_{t} \mathrm{z}_{t-j}\right]=E\left[\left(a_{t}-\theta_{1} a_{t-1}-\ldots-\theta_{q} a_{t-q}\right)\left(a_{t-j}-\theta_{1} a_{t-j-1}-\ldots-\theta_{q} a_{t-j-q}\right)\right]
$$


Como a série $a_{t}$ é um ruído branco, temos que

$$
E\left[a_{t} a_{t-j}\right]= \begin{cases}\sigma_{a}^{2}, & \text { se } j=0 \\ 0, & \text { se } j \neq 0\end{cases}
$$

Portanto, a variância do processo é

$$
\gamma_{0}=\left(1+\theta_{1}^{2}+\ldots+\theta_{q}^{2}\right) \sigma_{a}^{2}
$$

e

$$
\gamma_{j}=\left\{\begin{array}{l}
\left(-\theta_{j}+\theta_{1} \theta_{j+1}+\theta_{2} \theta_{j+2}+\ldots+\theta_{q} \theta_{q-j}\right) \sigma_{a}^{2}, j=1, \ldots, \mathrm{q} \\
0, j>\mathrm{q}
\end{array}\right.
$$

Das duas relações acima, obtemos a função de auto-correlação

$$
\rho_{\mathrm{j}}=\left\{\begin{array}{l}
\frac{-\theta_{j}+\theta_{1} \theta_{j+1}+\theta_{2} \theta_{j+2}+\ldots+\theta_{q} \theta_{q-j}}{1+\theta_{1}^{2}+\theta_{2}^{2}+\ldots+\theta_{q}^{2}}, j=1, \ldots, \mathrm{q} \\
0, j>\mathrm{q}
\end{array}\right.
$$

Vemos então que a função de auto-correlação para um processo MA(q) é igual a zero para $j>$ q. Esta propriedade é importante para identificar quando uma dada série é gerada por um processo MA(q). 


\subsection{Modelos Mistos Auto-regressivos - Médias Móveis}

Através da combinação de termos auto-regressivos e de médias móveis é formada uma classe importante de modelos para séries temporais.

Um modelo misto auto-regressivos - médias móveis contendo p termos AR e q termos MA, denotado por ARMA(p,q), é dado por

$$
\mathrm{z}_{t}=\phi_{1} \mathrm{z}_{t-1}+\phi_{2} \mathrm{z}_{t-2}+\ldots+\phi_{p} \mathrm{z}_{t-p}+a_{t}-\theta_{1} a_{t-1}-\theta_{2} a_{t-2}-\ldots-\theta_{q} a_{t-q}
$$

ou

$$
\phi(\mathrm{B}) \mathrm{z}_{t}=\theta(\mathrm{B}) a_{t}
$$

\subsubsection{Condições de Estacionariedade e Invertibilidade}

Temos que os termos de médias móveis que aparecem no lado direito da equação (2.12) acima, não afetam o argumento usado para estabelecer condições de estacionariedade de um processo auto-regressivo. Então, o processo $\phi(\mathrm{B}) \mathrm{z}_{\mathrm{t}}=\theta(\mathrm{B}) a_{t}$ será estacionário sob a condição de que o polinômio $\phi(B)=0$ tenha todas as raízes fora do círculo de raio unitário.

Analogamente, o processo será invertível se todas as raízes de $\theta(\mathrm{B})=0$ cairem fora do círculo de raio unitário.

\subsubsection{Função de Auto-correlação}

Consideremos a equação (2.12). Multiplicando ambos os membros por $z_{t-j}$ e tomando esperanças, obtemos 


$$
\gamma_{j}=\phi_{1} \gamma_{j-1}+\phi_{2} \gamma_{j-2}+\ldots+\phi_{p} \gamma_{j-p}+\gamma_{z a}(j)-\theta_{1} \gamma_{z a}(j-1)-\ldots-\theta_{q} \gamma_{z a}(j-\mathrm{q})
$$

onde $\gamma_{z a}(j)$ é a covariância cruzada definida por

$$
\gamma_{z a}(j)=E\left[a_{t} \mathrm{z}_{t-j}\right]
$$

Visto que $\mathrm{z}_{t-j}$ depende somente de choques $a_{t}$ ocorridos até o instante t-j, temos

$$
\gamma_{z a}(j)\left\{\begin{array}{l}
=0, \text { se } j>0 \\
\neq 0, \text { se } j \leq 0
\end{array}\right.
$$

Então, a equação (2.13) fica

$$
\gamma_{j}=\phi_{1} \gamma_{j-1}+\phi_{2} \gamma_{j-2}+\ldots+\phi_{p} \gamma_{j-p} \quad j>\mathrm{q}
$$

e com isso obtemos a função de auto-correlação

$$
\rho_{j}=\phi_{1} \rho_{j-1}+\phi_{2} \rho_{j-2}+\ldots+\phi_{p} \rho_{j-p} \quad j>\mathrm{q}
$$

de onde concluimos que para $j>\mathrm{q}$ as auto-correlações comportam-se como nos modelos auto-regressivos.

\subsection{Função de Auto-correlação Parcial}

Vimos que as funções de auto-correlação de cada processo visto anteriormente, apresentam características especiais. Assim, tendo um conjunto de dados observados, podemos estimar as funções de auto-correlação e, comparando o comportamento das funções 
estimadas com as características teóricas dessas funçōes, podemos escolher um ou mais modelos para descrever o processo que eventualmente gerou a série temporal.

Outro instrumento utilizado para facilitar a identificação do modelo, é a função de auto-correlação parcial (facp).

Denotemos por $\phi_{k j}$ o j-ésimo coeficiente de um modelo $\operatorname{AR}(\mathrm{k})$, de tal modo que $\phi_{k k}$ seja o último coeficiente. Da equação (2.8), temos que $\phi_{k j}$ satisfaz o conjunto de equações

$$
\rho_{j}=\phi_{k 1} \rho_{j-1}+\phi_{k 2} \rho_{j-2}+\ldots+\phi_{k k} \rho_{j-k} \quad j=1, \ldots, \mathrm{k}
$$

levando às equações de Yule-Walker (2.9), que podem ser escritas na forma:

$$
\left[\begin{array}{ccccc}
1 & \rho_{1} & \rho_{2} & \ldots & \rho_{k-1} \\
\rho_{1} & 1 & \rho_{1} & \cdots & \rho_{k-2} \\
\cdot & \cdot & \cdot & & \cdot \\
\cdot & \cdot & \cdot & \cdots & \cdot \\
\cdot & \cdot & \cdot & & \cdot \\
\rho_{k-1} & \rho_{k-2} & \rho_{k-3} & \cdots & 1
\end{array}\right]\left[\begin{array}{c}
\phi_{k 1} \\
\phi_{k 2} \\
\cdot \\
\cdot \\
\cdot \\
\phi_{k k}
\end{array}\right]=\left[\begin{array}{c}
\rho_{1} \\
\rho_{2} \\
\cdot \\
\cdot \\
\cdot \\
\rho_{k}
\end{array}\right]
$$

Resolvendo estas equações sucessivamente para $\mathrm{k}=1,2,3, \ldots$, teremos:

$$
\begin{aligned}
\phi_{11} & =\rho_{1} \\
\phi_{22} & =\frac{\left|\begin{array}{cc}
1 & \rho_{1} \\
\rho_{1} & \rho_{2}
\end{array}\right|}{\left|\begin{array}{cc}
1 & \rho_{1} \\
\rho_{1} & 1
\end{array}\right|}=\frac{\rho_{2}-\rho_{1}^{2}}{1-\rho_{1}^{2}} \\
\phi_{33} & =\frac{\left|\begin{array}{ccc}
1 & \rho_{1} & \rho_{1} \\
\rho_{1} & 1 & \rho_{2} \\
\rho_{2} & \rho_{1} & \rho_{3}
\end{array}\right|}{\left|\begin{array}{ccc}
1 & \rho_{1} & \rho_{2} \\
\rho_{1} & 1 & \rho_{1} \\
\rho_{2} & \rho_{1} & 1
\end{array}\right|}
\end{aligned}
$$


Em geral, para $\phi_{k k}$, o determinante no numerador tem os mesmos elementos que o denominador, mas com a última coluna trocada pelo vetor de auto-correlações.

Ao valor $\phi_{k k}$, denominamos função de auto-correlação parcial.

Para os processos vistos anteriormente, temos que:

i) um processo $\operatorname{AR}(\mathrm{p})$ tem facp $\phi_{k k} \neq 0$, se $\mathrm{k} \leq \mathrm{p}$ e $\phi_{k k}=0$, se $\mathrm{k}>\mathrm{p}$;

ii) um processo MA(q) é equivalente a um processo AR de ordem infinita, logo $\phi_{k k} \rightarrow 0$ quando $\mathrm{k} \rightarrow \infty$, então um processo MA(q) tem facp que se comportam de modo similar às funções de auto-correlações de um processo $\mathrm{AR}(\mathrm{p})$;

iii) um processo $\operatorname{ARMA}(p, q)$ tem facp que se comporta como a facp de um processo MA puro quando $\mathrm{k}>\mathrm{q}$.

Assim, como no caso das funções de auto-correlações, podemos usar as funções de auto-correlações amostrais $r_{j}$ definidas na seção 2.3 .2 e obter estimativas para $\phi_{k k}$. Em seguida, comparamos com as características dadas acima e escolhemos um modelo para a série dada.

Os modelos escolhidos devem ser parcimoniosos (no sentido que o número de parâmetros deve ser o menor possível) pois, para um número fixo de observações, quanto mais parâmetros em um modelo, menos eficiente é a estimação dos parâmetros.

Após a identificação do modelo para a série temporal dada, partimos para a estimação dos parâmetros. Empregaremos aqui métodos de estimativas de máxima verossimilhança, ou seja, métodos que obtêm os valores que maximizam a função de verossimilhança.

No capítulo seguinte, veremos como obter a função de verossimilhança para os modelos auto-regressivos e para os modelos de médias-móveis. 


\section{Capítulo 3 - Função de Verossimilhança Exata}

\subsection{Função de Verossimilhança Exata para um Processo Auto-regressivo}

Vamos supor aqui que uma dada série $\mathbf{z}_{n}^{\prime}=\left(z_{1}, z_{2}, \ldots, z_{n}\right)$ é gerada pelo modelo autoregressivo estacionário de ordem $\mathrm{p}$

$$
z_{t}-\phi_{1} z_{t-1}-\phi_{2} z_{t-2}-\ldots-\phi_{p} z_{t-p}=a_{t}
$$

onde $E\left[\mathbf{z}_{\imath}\right]=0$.

Assumindo que os $a$ 's e conseqüentemente os z's têm distribuição normal, temos que a função densidade de probabilidade conjunta dos z's é

$$
p\left(\mathbf{z}_{n} \mid \phi, \sigma_{a}^{2}\right)=\left(\frac{1}{2 \pi \sigma_{a}^{2}}\right)^{n / 2}\left|M_{n}^{(p)}\right|^{\overparen{1 / 2}} \cdot \exp \left\{-\frac{1}{2 \sigma_{a}^{2}} \mathbf{z}_{n} M_{J_{i}^{n}}^{(p)} \mathbf{z}_{n}\right\}
$$

onde $\phi=\left(\phi_{1}, \phi_{2}, \ldots, \phi_{p}\right)$ e a matriz $M_{n}^{(p)}$ é dada por

$$
M_{n}^{(p)}=\left\{m_{i j}^{(p)}\right\}=\left\{\gamma_{|i-j|}\right\}^{-1} \sigma_{a}^{2}=\left[\begin{array}{cccc}
\gamma_{0} & \gamma_{1} & \ldots & \gamma_{n-1} \\
\gamma_{1} & \gamma_{0} & \ldots & \gamma_{n-2} \\
\cdot & \cdot & & \cdot \\
\cdot & \cdot & & \cdot \\
\cdot & \cdot & & \cdot \\
\gamma_{n-1} & \gamma_{n-2} & \ldots & \gamma_{0}
\end{array}\right]^{-1} \sigma_{a}^{2}
$$

com $\gamma_{0}, \gamma_{1}, \ldots, \gamma_{n-1}$ as autocovâriancias teóricas do processo. 
Devido ao caráter reversível do processo geral, a matriz $M_{n}^{(p)}$ é duplamente simétrica, isto é, é simétrica em relação à ambas as suas diagonais principais.

Fazendo $\tau=\frac{1}{\sigma_{a}^{2}}$ a equação (3.1) fica

$$
p\left(\mathbf{z}_{n} \mid \phi, \tau\right)=\left(\frac{\tau}{2 \pi}\right)^{n / 2}\left|M_{n}^{(p)}\right|^{1 / 2} \exp \left\{-\frac{\tau}{2} \mathbf{z}_{n}^{\prime} M_{n}^{(p)} \mathbf{z}_{n}\right\}
$$

Observamos que, para $\mathbf{z}_{p}^{\prime}=\left(\mathbf{z}_{1}, \mathbf{z}_{2}, \ldots, \mathbf{z}_{p}\right)$ fixado, as séries $\left(a_{p+1}, a_{p+2}, \ldots, a_{n}\right)$ e $\left(\mathbf{z}_{p+1}, \mathbf{z}_{p+2}, \ldots, \mathbf{z}_{n}\right)$ são relacionadas pela seguinte transformação:

$$
\begin{array}{cc}
a_{p+1}=\mathrm{z}_{p+1}-\phi_{1} \mathrm{z}_{p}-\phi_{2} \mathrm{z}_{p-1}- & \ldots-\phi_{p} \mathrm{z}_{1} \\
\cdot & \cdot \\
\cdot & \cdot \\
\cdot & \cdot \\
a_{n}=\mathrm{z}_{n}-\phi_{1} \mathrm{z}_{n-1}-\phi_{2} \mathrm{z}_{n-2}-\ldots-\phi_{p} \mathrm{z}_{n-p}
\end{array}
$$

Assumindo que os $a_{p+1}, a_{p+2}, \ldots, a_{n}$ têm distribuição normal, temos

$$
p\left(a_{p+1}, a_{p+2}, \ldots, a_{n} \mid \mathbf{z}_{p}, \phi, \tau\right)=\left(\frac{\tau}{2 \pi}\right)^{(n-p) / 2} \exp \left\{-\frac{\tau}{2} \sum_{t=p+1}^{n} a_{t}^{2}\right\}
$$

e, como o jacobiano da transformação é unitário, segue que

$$
p\left(\mathbf{z}_{p+1}, \mathbf{z}_{p+2}, \ldots, \mathbf{z}_{n} \mid \mathbf{z}_{p}, \phi, \tau\right)=\left(\frac{\tau}{2 \pi}\right)^{(n-p) / 2} \exp \left\{-\frac{\tau}{2} \sum_{t=p+1}^{n}\left(\mathbf{z}_{t}-\phi_{1} \mathbf{z}_{t-1}-\ldots-\phi_{p} \mathbf{z}_{t-p}\right)^{2}\right\}
$$

A função acima é chamada função de verossimilhaça aproximada (ou condicional) por estar condicionada em $\mathbf{z}_{p}$. Podemos obter a função de verossimilhança exata, para os dados $\left(\mathrm{z}_{1}, \mathrm{z}_{2}, \ldots, \mathrm{z}_{\mathrm{n}}\right)$, através da seguinte equação: 


$$
p\left(\mathbf{z}_{n} \mid \phi, \tau\right)=p\left(\mathbf{z}_{p+1}, \mathbf{z}_{p+2}, \ldots, \mathbf{z}_{n} \mid \mathbf{z}_{p}, \phi, \tau\right) p\left(\mathbf{z}_{p} \mid \phi, \tau\right)
$$

\section{Como}

$$
p\left(\mathbf{z}_{p} \mid \phi, \tau\right)=\left(\frac{\tau}{2 \pi}\right)^{p / 2}\left|M_{p}^{(p)}\right|^{1 / 2} \exp \left\{-\frac{\tau}{2} \mathbf{z}_{p}^{\prime} \boldsymbol{M}_{p}^{(p)} \mathbf{z}_{p}\right\}
$$

onde $M_{p}^{(p)}$ é a matriz de correlação entre as p-primeiras observações, segue que

$$
p\left(\mathbf{z}_{\mathrm{n}} \mid \phi, \tau\right)=\left(\frac{\tau}{2 \pi}\right)^{\pi / 2}\left|M_{p}^{(p)}\right|^{1 / 2} \exp \left\{-\frac{\tau}{2} S(\phi)\right\}
$$

onde

$$
S(\phi)=\sum_{i=1}^{p} \sum_{j=1}^{p} m_{i j}^{(p)} \mathbf{z}_{i} \mathbf{z}_{j}+\sum_{t=p+1}^{n}\left(\mathrm{z}_{t}-\phi_{1} \mathrm{z}_{t-1}-\ldots-\phi_{p} \mathrm{z}_{t-p}\right)^{2}
$$

Veremos agora como calcular os elementos de $M_{p}^{(p)}$. Seja $n=p+1$, de modo que

$$
\mathbf{z}_{p+1}^{\prime} \boldsymbol{M}_{p+1}^{(p)} \mathbf{z}_{p+1}=\sum_{i=1}^{p} \sum_{j=1}^{p} m_{i j}^{(p)} \mathrm{z}_{i} z_{j}+\left(\mathrm{z}_{\mathrm{p}+1}-\phi_{1} \mathrm{z}_{\mathrm{p}}-\phi_{2} \mathrm{z}_{\mathrm{p}-1}-\ldots-\phi_{p} \mathrm{z}_{1}\right)^{2}
$$

Então

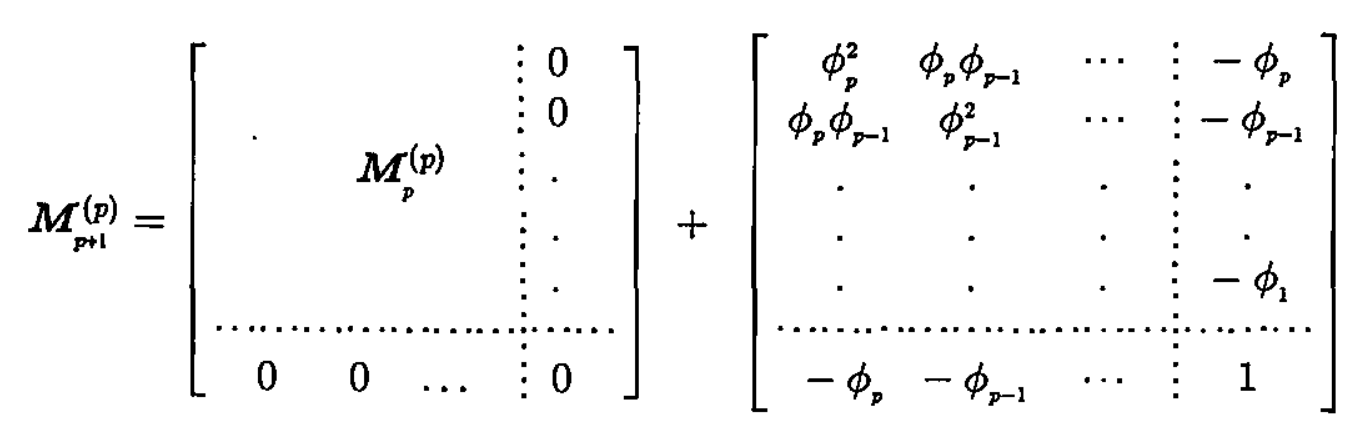


e os elementos $m_{i j}^{(p)}$ de $M_{p}^{(p)}$ podem ser deduzidos usando o fato que ambos $M_{p}^{(p)}$ e $M_{p+1}^{(p)}$ são duplamente simétricos.

Assim, por exemplo, para $\mathrm{p}=1$

$$
M_{2}^{(1)}=\left[\begin{array}{cc}
m_{11}^{(1)}+\phi_{1}^{2} & -\phi_{1} \\
-\phi_{1} & 1
\end{array}\right]=\left[\begin{array}{cc}
1 & -\phi_{1} \\
-\phi_{1} & m_{11}^{(1)}+\phi_{1}^{2}
\end{array}\right]
$$

Igualando os elementos nas duas matrizes, obtemos

$$
M_{1}^{(1)}=m_{11}^{(1)}=1-\phi_{1}^{2}
$$

e

$$
\left|M_{1}^{(1)}\right|=1-\phi_{1}^{2}
$$

Então, a função densidade de probabilidade para $\mathrm{p}=1$ é

$$
p\left(\mathbf{z}_{\mathrm{n}} \mid \phi, \tau\right)=\left(\frac{\tau}{2 \pi}\right)^{n / 2}\left(1-\phi_{1}^{2}\right)^{1 / 2} \exp \left[-\frac{\tau}{2}\left\{\left(1-\phi_{1}^{2}\right) \mathbf{z}_{1}^{2}+\sum_{t=2}^{n}\left(\mathrm{z}_{t}-\phi_{1} \mathbf{z}_{t-1}\right)^{2}\right\}\right]
$$

Analogamente, para $\mathrm{p}=2$, temos

$$
M_{2}^{(2)}=\left[\begin{array}{cc}
1-\phi_{2}^{2} & -\phi_{1}\left(1+\phi_{2}\right) \\
-\phi_{1}\left(1+\phi_{2}\right) & 1-\phi_{2}^{2}
\end{array}\right]
$$

e

$$
\left|M_{2}^{(2)}\right|=\left(1+\phi_{2}\right)^{2}\left\{\left(1-\phi_{2}\right)^{2}-\phi_{1}^{2}\right\}
$$


Portanto,

$$
\begin{aligned}
p\left(\mathbf{z}_{n} \mid \phi, \tau\right)= & \left(\frac{\tau}{2 \pi}\right)^{n / 2}\left[\left(1+\phi_{2}\right)^{2}\left\{\left(1-\phi_{2}\right)^{2}-\phi_{1}^{2}\right\}\right]^{\frac{1}{2}} \cdot \\
& \exp \left[-\frac{\tau}{2}\left\{\left(1-\phi_{2}^{2}\right)\left(\mathbf{z}_{1}^{2}+\mathrm{z}_{2}^{2}\right)-2 \phi_{1}\left(1+\phi_{2}\right) \mathrm{z}_{1} \mathrm{z}_{2}+\sum_{t=3}^{n}\left(\mathrm{z}_{t}-\phi_{1} \mathrm{z}_{t-1}-\phi_{2} \mathrm{z}_{t-2}\right)^{2}\right\}\right]
\end{aligned}
$$

Observamos que os elementos de $M_{p}^{(p)}$ gerados pelo processo acima são quadráticos nos $\phi$ 's. Assim, de (3.5), temos que $S(\phi)=\mathbf{z}_{n}^{\prime} M_{n}^{(p)} \mathbf{z}_{n}$ é uma forma quadrática não somente nos z's, mas também nos parâmetros $\phi$.

Definindo o vetor $\phi_{u}^{\prime}=\left(1, \phi_{1}, \phi_{2}, \ldots, \phi_{p}\right)$, temos que, para alguma matriz $D$ de ordem $(p+1) \times(p+1)$ cujos elementos são funções quadráticas dos $z$ 's,

$$
\mathbf{z}_{n}^{\prime} M_{n}^{(p)} \mathbf{z}_{n}=\phi_{u}^{\prime} D \phi_{u}
$$

Seja

$$
D=\left[\begin{array}{ccccc}
D_{11} & -D_{12} & -D_{13} & \ldots & -D_{1, p+1} \\
-D_{12} & D_{22} & D_{23} & \ldots & D_{2, p+1} \\
\cdot & \cdot & \cdot & \cdot & \cdot \\
\cdot & \cdot & \cdot & \cdot & \cdot \\
-D_{1, p+1} & D_{2, p+1} & D_{3, p+1} & \cdots & D_{p+1, p+1}
\end{array}\right]
$$

A inspeção de (3.5) mostra que os elementos $D_{i j}$ são somas "simétricas" de quadrados e produtos espaçados, definidos por

$$
D_{i j}=D_{j i}=z_{i} z_{j}+z_{i+1} z_{j+1}+\ldots+z_{n+1-j} z_{n+1-i}
$$

onde a soma acima possui $n-(i-1)-(j-1)$ termos. 
Podemos agora escrever a densidade de probabilidade exata e, conseqüientemente, a verossimilhança exata, como:

$$
p\left(\mathbf{z}_{n} \mid \phi, \tau\right)=L\left(\phi, \tau \mid \mathbf{z}_{n}\right)=\left(\frac{\tau}{2 \pi}\right)^{n / 2}\left|M_{p}^{(p)}\right|^{1 / 2} \exp \left\{-\frac{\tau}{2} S(\phi)\right\}
$$

onde

$$
S(\phi)=\mathrm{z}_{p}, M_{p}^{(p)} \mathbf{z}_{p}+\sum_{t=p+1}^{n}\left(\mathrm{z}_{t}-\phi_{1} \mathrm{z}_{t-1}-\ldots-\phi_{p} \mathrm{z}_{t-p}\right)^{2}=\phi_{u}^{\prime} D \phi_{u}
$$

Por conveniência, costuma-se trabalhar com a função log-verossimilhança

$$
l\left(\phi, \tau \mid \mathbf{z}_{n}\right)=\ln \left\{L\left(\phi, \tau \mid \mathbf{z}_{n}\right)\right\}=\frac{n}{2} \ln (\tau)+\frac{1}{2} \ln \left|M_{p}^{(p)}\right|-\frac{\tau}{2} S(\phi)
$$

\section{Estimativas de Máxima Verossimilhança}

Os valores dos parâmetros que maximizam a função de verossimilhança, ou equivalentemente a função log-verossimilhança, são chamados estimativas de máxima verossimilhança.

Diferenciando a função (3.9) em relação a $\tau$ e em relação a cada um dos $\phi$ 's, obtemos

$$
\begin{gathered}
\frac{\delta l}{\delta \tau}=\frac{n}{2 \tau}-\frac{1}{2} S(\phi) \\
\frac{\delta l}{\delta \phi_{j}}=M_{j}+\tau\left\{D_{1, j+1}-\phi_{1} D_{2, j+1}-\ldots-\phi_{p} D_{p+1, j+1}\right\} \quad j=1,2, \ldots, p
\end{gathered}
$$

onde

$$
M_{j}=\frac{\delta\left\{\frac{1}{2} \ln \left|M_{p}^{(p)}\right|\right\}}{\delta \phi_{j}}
$$


Igualando estas expressões a zero e resolvendo a equação resultante, podemos obter estimativas de máxima verossimilhança.

Denotaremos aqui as estimativas dos parâmetros por $\widehat{\tau}$ e $\widehat{\phi}$.

Uma estimativa para o parâmetro $\tau$ é obtida diretamente da expressão (3.10)

$$
\widehat{\tau}^{-1}=\frac{S(\widehat{\phi})}{n}
$$

Se para a obtenção das estimativas dos parâmetros $\phi$ 's simplesmente igualarmos a equação (3.11) a zero, obtemos uma expressão complicada devido ao fator $M_{j}$.

No próximo capítulo, na seção 4.1 , veremos três métodos que evitam o cálculo deste fator.

\subsection{Função de Verossimilhança Exata para um Processo de Médias- móveis}

Consideremos o modelo de médias-móveis invertível de ordem q, dado por

$$
\mathrm{z}_{\mathrm{t}}=a_{t}-\theta_{1} a_{t-1}-\theta_{2} a_{t-2}-\ldots-\theta_{q} a_{t-q}
$$

onde os z's têm média $\mu=0$, e suponhamos que uma dada série, $\left.\mathbf{z}_{n}^{\prime}\right)^{\prime}=\left(z_{1}, z_{2}, \ldots, z_{n}\right)^{\prime}$ é gerada por este modelo.

Supondo que os $a$ 's e, portanto, os z's têm distribuição normal, a função densidade de probabilidade conjunta dos z's pode ser escrita como:

$$
p\left(\mathbf{z}_{n} \mid \boldsymbol{\theta}, \sigma_{a}^{2}\right)=\left(\frac{1}{2 \pi \sigma_{a}^{2}}\right)^{n / 2}\left|M_{n}^{(q)}\right|^{1 / 2} \exp \left\{-\frac{1}{2 \sigma_{a}^{2}} \mathbf{z}_{n}^{\prime} M_{n}^{(q)} \mathbf{z}_{n}\right\}
$$


onde $\theta=\left(\theta_{1}, \theta_{2}, \ldots, \theta_{q}\right)$ e $\left(M_{n}^{(q)}\right)^{-1} \sigma_{a}^{2}$ representa a matriz de covariância dos z's para um processo MA(q).

Antes de definir a função de verossimilhança, vamos obter uma expressão mais conveniente para a densidade acima. Através da equação (3.13), fazendo $t \doteq 1,2, \ldots, n$, podemos obter as $n$ equações:

$$
\begin{aligned}
& \mathbf{z}_{\mathbf{1}}=a_{1}-\theta_{1} a_{0} \quad-\theta_{2} a_{-1}-\ldots-\theta_{q} a_{1-q} \\
& z_{2}=a_{2}-\theta_{1} a_{1}-\theta_{2} a_{0}-\ldots-\theta_{q} a_{2-q} \\
& z_{n}=a_{n}-\theta_{1} a_{n-1}-\theta_{2} a_{n-2}-\ldots-\theta_{q} a_{n-q}
\end{aligned}
$$

Vamos reescrever estas $n$ equações na seguinte forma matricial:

$$
\mathrm{z}_{\mathrm{n}}=G a+F a_{*}
$$

onde:

- $\mathbf{z}_{n}^{\prime}=\left(z_{1}, z_{2}, \ldots, z_{n}\right), a^{\prime}=\left(a_{1}, a_{2}, \ldots, a_{n}\right)$ e $a_{*}^{\prime}=\left(a_{1-q}, a_{2-q}, \ldots, a_{0}\right)$ é um vetor q-dimensional dos valores preliminares do ruído;

- $G$ é uma matriz triangular inferior de tamanho $n \times n$ formada por 1's na diagonal principal, $-\theta_{1}$ na primeira subdiagonal, $-\theta_{2}$ na segunda subdiagonal, e assim por diante, $\operatorname{com} \theta_{i}=0$, para $i>\mathrm{q}$;

- $F$ é uma matriz de tamanho $n \times \mathrm{q}$ da forma $F=\left(B_{q}^{\prime}, O^{\prime}\right)^{\prime}$, onde

$$
\boldsymbol{B}_{q}=-\left[\begin{array}{cccc}
\theta_{q} & \theta_{q-1} & \ldots & \theta_{1} \\
0 & \theta_{q} & \ldots & \theta_{2} \\
\vdots & \vdots & \ldots & \vdots \\
0 & 0 & \ldots & \theta_{q}
\end{array}\right]
$$

é uma matriz quadrada de ordem q e $O$ uma matriz nula de ordem $(n-\mathrm{q})$ x q. 
Agora, considerando o vetor formado pelos ruídos $\left(a^{\prime}, a_{*}^{\prime}\right)$, temos que a distribuição conjunta desses $n+q$ valores é dada por:

$$
p\left(a, a_{*} \mid \sigma_{a}^{2}\right)=\left(\frac{1}{2 \pi \sigma_{a}^{2}}\right)^{(n+q) / 2} \exp \left\{-\frac{1}{2 \sigma_{a}^{2}}\left(a^{\prime} a+a_{*}^{\prime} a_{*}\right)\right\}
$$

Observamos que a transformação de $\left(a, a_{*}\right)$ em $\left(\mathbf{z}_{n}, a_{*}\right)$ tem jacobiano unitário e é dada pela relação $a=G^{-1}\left(\mathbf{z}_{n}-F a_{*}\right)$. Usando esse fato, e fazendo $\tau=\frac{1}{\sigma_{a}^{2}}$, podemos escrever a densidade conjunta de $\mathbf{z}_{n}$ e $a_{*}$ como:

$$
p\left(\mathbf{z}_{n}, a_{*} \mid \boldsymbol{\theta}, \tau\right)=\left(\frac{\tau}{2 \pi}\right)^{(n+q) / 2} \exp \left\{-\frac{\tau}{2} \mathrm{~S}\left(\boldsymbol{\theta}, a_{*}\right)\right\}
$$

onde

$$
\mathrm{S}\left(\theta, a_{*}\right)=\left(\mathrm{z}_{n}-F a_{*}\right)^{\prime} G^{\prime-1} G^{-1}\left(\mathbf{z}_{n}-F a_{*}\right)+a_{*}^{\prime} a_{*}
$$

Utilizando a teoria dos mínimos quadrados generalizada, pode ser mostradoque se $\widehat{a}_{*}$ é o vetor que minimiza $S\left(\theta, a_{*}\right)$, então $\widehat{a}_{*}=D^{-1} F^{\prime} G^{\prime^{-1}} G^{-1} \mathbf{z}_{n}$ onde $D=I_{q}+F^{\prime} G^{\prime^{-1}} G^{-1} \not{ }^{\prime}$. Além disso, teremos que:

$$
\mathrm{S}\left(\theta, a_{*}\right)=\mathrm{S}(\theta)+\left(a_{*}-\widehat{a}_{*}\right)^{\prime} D\left(a_{*}-\widehat{a}_{*}\right)
$$

onde

$$
\mathrm{S}(\theta)=\mathrm{S}\left(\theta, \widehat{a}_{*}\right)=\left(\mathrm{z}_{n}-F \widehat{a}_{*}\right)^{\prime} G^{\prime-1} G^{-1}\left(\mathbf{z}_{n}-F \widehat{a}_{*}\right)+\widehat{a}_{*}^{\prime} \widehat{a}_{*}
$$

Portanto, substituindo em (3.15) temos 
$p\left(\mathbf{z}_{n}, a_{*} \mid \boldsymbol{\theta}, \tau\right)=\left(\frac{\tau}{2 \pi}\right)^{(n+q) / 2} \exp \left\{-\frac{\tau}{2}\left[\mathbf{S}(\theta)+\left(a_{*}-\widehat{a}_{*}\right)^{\prime} D\left(a_{*}-\widehat{a}_{*}\right)\right]\right\}^{\prime}$

Visto que podemos fatorar a distribuição conjunta acima como o produto

$$
p\left(\mathbf{z}_{n}, \boldsymbol{a}_{*} \mid \boldsymbol{\theta}, \tau\right)=p\left(\mathbf{z}_{\mathbf{n}} \mid \boldsymbol{\theta}, \tau\right) p\left(\boldsymbol{a}_{*} \mid \mathbf{z}_{\mathbf{n}}, \boldsymbol{\theta}, \tau\right)
$$

segue que:

$$
p\left(\boldsymbol{a}_{*} \mid \mathbf{z}_{n}, \boldsymbol{\theta}, \tau\right)=\left(\frac{\tau}{2 \pi}\right)^{q / 2}|D|^{n / / 2} \cdot \exp \left\{-\frac{\tau}{2}\left[\left(\boldsymbol{a}_{*}-\widehat{a}_{*}\right)^{\prime} D\left(a_{*}-\widehat{a}_{*}\right)\right]\right\}
$$

$\mathrm{e}$

$$
p\left(\mathbf{z}_{n} \mid \boldsymbol{\theta}, \tau\right)=\left(\frac{\tau}{2 \pi}\right)^{n / 2}|\stackrel{D}{P}|^{-n_{1} / 2} \cdot \exp \left\{-\frac{\tau_{1}}{2} \mathrm{~S}(\boldsymbol{\theta})\right\}
$$

Agora, analisando a expressão (3.17), podemos deduzir que $\widehat{a}_{*}=E\left[\boldsymbol{a}_{*} \mid \mathbf{z}_{n}, \theta\right]$. Para simplificar, denotaremos esta esperança condicional por $\left[a_{*}\right]$. Daí, usando o fato que $a=G^{-1}\left(\mathbf{z}_{n}-F a_{*}\right)$, concluímos que

$$
[a]=G^{-1}\left(\mathbf{z}_{n}-F\left[a_{*}\right]\right)
$$

é a esperança condicional de $a$ dado $\mathbf{z}_{n}$ e $\theta$ e, substituindo em (3.16), vem

$$
S(\theta)=[a]^{\prime}[a]+\left[a_{*}\right]^{\prime}\left[a_{*}\right]=\sum_{t=1-q}^{n}\left[a_{t}\right]^{2}
$$

onde $\left[a_{t}\right]=E\left[a_{t} \mid \mathbf{z}_{n}, \boldsymbol{\theta}\right]$.

Conseqüentemente, através das equações (3.18) e (3.19), obtemos finalmente a seguinte expressão para a função de verossimilhança exata (ou incondicional) para um processo $\mathrm{MA}(\mathrm{q})$ : 


$$
L\left(\theta, \tau \mid \mathbf{z}_{n}\right)=\left(\frac{\tau}{2 \pi}\right)^{n / 2}|D|^{-1 / 2} \exp \left\{-\frac{\tau}{2} \sum_{t=1-q}^{n}\left[a_{t}\right]^{2}\right\}
$$

O cálculo dos estimadores de máxima verossimilhança para os parâmetros $\theta$ s $\delta$ pode ser feito numericamente.

No capítulo 4 apresentamos alguns métodos que possibilitam obter as estimativas de máxima verossimilhança.

Destacamos aqui que o estimador de $\theta$ que maximiza (3.20) é função das estimativas de mínimos quadrados $\widehat{a}_{*}$. 


\section{Capítulo 4 - Métodos Numéricos para Estimativas de Máxima Verossimilhança}

\subsection{Métodos Numéricos para Estimativas de Máxima Verossimilhança para Modelos AR(p)}

Exporemos aqui três métodos para a obtenção das estimativas dos parâmetros de um modelo $A R(p)$. O primeiro método utiliza estimativas mínimos quadrados obtidas resolvendose um sistema linear de $\mathrm{p}$ equações. O segundo método obtém uma aproximação para as derivadas $\frac{\delta l}{\delta \phi_{j}}$ da expressão (3.11) e calcula as estimativas $\widehat{\phi}$ de $\phi$ através de um sistema linear de equações similar ao do primeiro método. O último método propõe um algoritmo iterativo, utilizando expressões mais simples para as derivadas da função log-verossimilhança.

\subsubsection{Estimativas Mínimos Quadrados}

Analisando a equação (3.7), observamos que para amostras moderadas ou grandes o valor de $\left|M_{p}^{(p)}\right|$ é pequeno em comparação a $S(\phi)$, visto que o valor esperado de $S(\phi)$ é proporcional a $n$ e $\left|M_{p}^{(p)}\right|$ é independente de $n$.

Então, ignorando a influência deste termo, temos

$$
l\left(\phi, \tau \mid \mathbf{z}_{n}\right) \simeq \frac{n}{2} \ln (\tau)-\frac{\tau}{2} S(\phi)
$$


e as estimativas $\widehat{\phi}$ de $\phi$ obtidas pela maximização de (4.1) são as estimativas mínimos quadrados obtidas minimizando $S(\phi)$. De (3.8) temos que $S(\phi)=\phi_{u}^{\prime} D \phi_{u}$ onde $D$ é a matriz definida em (3.6). Calculando esta expressão, diferenciando em relação a cada um dos $\phi_{i}$ 's e igualando a zero, os valores de minimização obtidos são dados pelo sistema de equações:

$$
\begin{array}{cc}
D_{12}=\widehat{\phi}_{1} D_{22}+\widehat{\phi}_{2} D_{23}+\ldots+\widehat{\phi}_{p} D_{2, p+1} \\
D_{13}=\widehat{\phi}_{1} D_{23}+\widehat{\phi}_{2} D_{33}+\ldots+\widehat{\phi}_{p} D_{3, p+1} \\
\cdot & \cdot \\
\cdot & \cdot \\
D_{1, p+1}=\widehat{\phi}_{1} D_{2, p+1}+\widehat{\phi}_{2} D_{3, p+1}+\ldots+\widehat{\phi}_{p} D_{p+1, p+1}
\end{array}
$$

Em notação matricial, podemos escrever o sistema acima na forma

$$
\mathbf{d}=\mathbf{D}_{\mathbf{p}} \widehat{\phi}
$$

de modo que

$$
\widehat{\phi}=D_{p}^{-1} \mathbf{d}
$$

\subsubsection{Aproximação das Estimativas de Máxima Verossimilhança}

Vimos anteriormente na expressão (3.11) que

$$
\frac{\delta l}{\delta \phi_{j}}=M_{j}+\tau\left\{D_{1, j+1}-\phi_{1} D_{2, j+1}-\ldots-\phi_{p} D_{p+1, j+1}\right\} \quad j=1,2, \ldots, \mathrm{p}
$$

Então, dividindo por $\tau$ e tomando esperanças obtemos

$$
\begin{aligned}
\frac{M_{j}}{\tau} & +(n-j) \gamma_{j}-(n-j-1) \phi_{1} \gamma_{j-1}-(n-j-2) \phi_{2} \gamma_{j-2}-\ldots \\
& -(n-j-p) \phi_{p} \gamma_{j-p}=0
\end{aligned}
$$


usando o fato que para os valores de maximização temos que $E\left[\delta l / \delta \phi_{j}\right]=0$.

Na seção 2.3.2, vimos que num processo autoregressivo as autocovariâncias são dadas por

$$
\gamma_{j}=\phi_{1} \gamma_{j-1}+\phi_{2} \gamma_{j-2}+\ldots+\phi_{p} \gamma_{j-p} \quad j>0
$$

que pode ser escrito na forma

$$
\gamma_{j}-\phi_{1} \gamma_{j-1}-\phi_{2} \gamma_{j-2}-\ldots-\phi_{p} \gamma_{j-p}=0 \quad j>0
$$

Agora, multiplicando esta expressão por $n$ e subtraindo o resultado de (4.3), obtemos

$$
\frac{M_{j}}{\tau}=j \gamma_{j}-(j+1) \phi_{1} \gamma_{j-1}-(j+2) \phi_{2} \gamma_{j-2}-\ldots-(j+p) \phi_{p} \gamma_{j-p}
$$

Tomando $D_{i+1, j+1} /(n-j-i)$ como uma estimativa de $\gamma_{\mid j-i j}$, uma estimativa natural de $M_{j} / \tau$ é

$$
j \frac{D_{1, j+1}}{n-j}-(j+1) \phi_{1} \frac{D_{2, j+1}}{n-j-1}-\ldots-(j+p) \phi_{p} \frac{D_{p+1, j+1}}{n-j-p}
$$

Substituindo esta estimativa em (3.11), segue que

$$
\frac{\delta l}{\delta \phi_{j}} \simeq n \tau\left\{\frac{D_{1, j+1}}{n-j}-\phi_{1} \frac{D_{2, j+1}}{n-j-1}-\ldots-\phi_{p} \frac{D_{p+1, j+1}}{n-j-p}\right\} \quad j=1,2, \ldots, \mathrm{p}
$$

o que leva a um conjunto de equações lineares da forma (4.2), mas agora com

$$
D_{i j}^{*}=n D_{i j} /\{n-(i-1)-(j-1)\}
$$

no lugar de $D_{i j}$. 


\subsubsection{ARMLE}

Este método, proposto por [Miller - 1995], é baseado em dois teoremas que propõem expressões mais simples para as derivadas da função log-verossimilhança dada em (3.9).

Teorema 1: As derivadas da função log-verossimilhança em relação aos parâmetros $\tau$ e $\phi$ são:

$$
\begin{aligned}
& \frac{\delta l}{\delta \tau}=\frac{n}{2 \tau}-\frac{1}{2} S(\phi) \\
& \frac{\delta l}{\delta \phi_{i}}=\tau\left\{D_{i+1,1}-\sum_{j=1}^{p} \phi_{j}\left(D_{i+1, j+1}+j \gamma_{j-i}\right)\right\} \quad i=1,2, \ldots, \mathrm{p}
\end{aligned}
$$

Prova: Obtemos a equação (4.4) pela simples diferenciação da função log-verossimilhança em relação a $\tau$. Provemos então a segunda equação. Temos que

$$
\frac{\delta l}{\delta \phi_{i}}=\frac{1}{2} \frac{\delta}{\delta \phi_{i}} \ln \left|M_{p}^{(p)}\right|-\frac{\tau}{2} \frac{\delta}{\delta \phi_{i}} S(\phi) \quad i=1,2, \ldots, \mathrm{p}
$$

Desde que (3.7) é uma função densidade,

$$
\int p\left(\mathbf{z}_{n} \mid \phi, \tau\right) d \mathbf{z}_{n}=\int\left(\frac{\tau}{2 \pi}\right)^{n / 2}\left|M_{p}^{(p)}\right|^{1 / 2} \exp \left\{-\frac{\tau}{2} S(\phi)\right\} d \mathbf{z}_{n}=1
$$

o que implica

$$
\left(\frac{\tau}{2 \pi}\right)^{n / 2}\left|M_{p}^{(p)}\right|^{1 / 2}=\left[\int \exp \left\{-\frac{\tau}{2} S(\phi)\right\} d \mathbf{z}_{n}\right]^{-1}
$$

Aplicando logaritmo em ambos os lados desta expressão, vem 


$$
\frac{n}{2} \ln \left(\frac{\tau}{2 \pi}\right)+\frac{1}{2} \ln \left|\boldsymbol{M}_{\mathrm{p}}^{(p)}\right|=-\ln \left[\int \exp \left\{-\frac{\tau}{2} S(\boldsymbol{\phi})\right\} d \mathbf{z}_{n}\right]
$$

e diferenciando cada lado em relação a $\phi_{i}$

$$
\begin{aligned}
\frac{1}{2} \frac{\delta}{\delta \phi_{i}} \ln \left|\boldsymbol{M}_{p}^{(p)}\right| & =\frac{\int\left\{(\tau / 2)\left(\delta / \delta \phi_{i}\right) S(\phi)\right\} \exp \left\{-\frac{\tau}{2} S(\phi)\right\} d \mathbf{z}_{n}}{\int \exp \left\{-\frac{\tau}{2} S(\phi)\right\} d \mathbf{z}_{n}} \\
& =\frac{\tau}{2} E\left\{\frac{\delta}{\delta \phi_{i}} S(\phi)\right\}
\end{aligned}
$$

Substituindo (4.7) em (4.6), obtemos

$$
\frac{\delta l}{\delta \phi_{i}}=\frac{\tau}{2}\left[E\left\{\frac{\delta}{\delta \phi_{i}} S(\phi)\right\}-\frac{\delta}{\delta \phi_{i}} S(\phi)\right] \quad i=1,2, \ldots, \mathrm{p}
$$

Usando o fato que (3.8) pode ser escrito na forma

$$
S(\phi)=D_{11}-2 \sum_{j=1}^{p} \phi_{j} D_{1, j+1}+\sum_{j=1}^{p} \sum_{k=1}^{p} \phi_{j} D_{j+1, k+1} \phi_{k}
$$

podemos verificar que

$$
\frac{\delta}{\delta \phi_{i}} S(\phi)=-2\left\{D_{i+1,1}-\sum_{j=1}^{p} \phi_{j} D_{i+1, j+1}\right\} \quad i=1,2, \ldots, \mathrm{p}
$$

$$
\begin{aligned}
& E\left\{\frac{\delta}{\delta \phi_{i}} S(\phi)\right\}=-2\left\{E\left(D_{i+1,1}\right)-\sum_{j=1}^{p} \phi_{j} E\left(D_{i+1 j+1}\right)\right\} \\
&=-2\left\{(n-i) \gamma_{i}-\sum_{j=1}^{p} \phi_{j}(n-i-j) \gamma_{j-i}\right\} \\
&=-2\left\{(n-i) \gamma_{i}-(n-i) \sum_{j=1}^{p} \phi_{j} \gamma_{j-i}+\sum_{j=1}^{p} j \phi_{j} \gamma_{j-i}\right\} \\
& i=1,2, \ldots, \mathrm{p}
\end{aligned}
$$


Da equação de Yule-Walker para modelos AR(p), temos

$$
\gamma_{i}=\sum_{j=1}^{p} \phi_{j} \gamma_{j-i} \quad i>0
$$

Logo,

$$
E\left\{\frac{\delta}{\delta \phi_{i}} S(\phi)\right\}=-2 \sum_{j=1}^{p} j \phi_{j} \gamma_{j-i} \quad i=1,2, \ldots, \mathrm{p}
$$

Finalmente, substituindo (4.10) e (4.11) em (4.8), obtemos

$$
\begin{aligned}
\frac{\delta l}{\delta \phi_{i}} & =\frac{\tau}{2}\left[-2 \sum_{j=1}^{p} j \phi_{j} \gamma_{j-i}+2\left\{D_{i+1,1}-\sum_{j=1}^{p} \phi_{j} D_{i+1, j+1}\right\}\right] \\
& =\tau\left\{D_{i+1,1}-\sum_{j=1}^{p} \phi_{j}\left(D_{i+1, j+1}+j \gamma_{j-i}\right)\right\} \quad i=1,2, \ldots, \mathrm{p}
\end{aligned}
$$

Teorema 2: As equaçōes de verossimilhança obtidas igualando as expressões (4.4) e (4.5) a zero podem ser expressas na forma

$$
\begin{aligned}
& D_{11}=\sum_{j=1}^{p} \phi_{j}\left(D_{1 j+1}+j \gamma_{j}\right)+\frac{n}{\tau} \\
& D_{i+1,1}=\sum_{j=1}^{p} \phi_{j}\left(D_{i+1, j+1}+j \gamma_{j-i}\right) \quad i=1,2, \ldots, \mathrm{p}
\end{aligned}
$$

Prova: A equação (4.13) é oḅtida simplesmente igualando a expressão (4.5) a zero. Vamos então provar a equação (4.12). Igualando (4.4) a zero, obtemos

$$
S(\phi)=\frac{n}{\tau}
$$


e reescrevendo (4.9) na forma

$$
S(\phi)=\left\{D_{11}-\sum_{j=1}^{p} \phi_{j} D_{1, j+1}\right\}-\sum_{i=1}^{p} \phi_{i}\left\{D_{i+1,1}-\sum_{j=1}^{p} \phi_{j} D_{i+1, j+1}\right\}
$$

segue que

$$
D_{11}=\sum_{j=1}^{p} \phi_{j} D_{1, j+1}+\sum_{i=1}^{p} \phi_{i}\left\{D_{i+1,1}-\sum_{j=1}^{p} \phi_{j} D_{i+1, j+1}\right\}+\frac{n}{\tau}
$$

Da equação (4.13), vem

$$
D_{i+1,1}-\sum_{j=1}^{p} \phi_{j} D_{i+1, j+1}=\sum_{j=1}^{p} j \phi_{j} \gamma_{j-i} \quad i=1,2, \ldots, \mathrm{p}
$$

e, portanto

$$
D_{11}=\sum_{j=1}^{p} \phi_{j}\left(D_{1 j+1}+j \gamma_{j}\right)+\frac{n}{\tau}
$$

A seguir é apresentado um algoritmo iterativo para obter as estimativas de máxima verossimilhança de $\tau$ e $\phi$.

\section{Algoritmo:}

1) Calcule a matriz $D$ e as estimativas iniciais de $\gamma_{p}=\left(\gamma_{1}, \gamma_{2}, \ldots, \gamma_{p}\right)$ usando autocovariâncias amostrais.

2) Usando $D$ e as estimativas de $\gamma_{p}$ correntes, resolver (4.12) e (4.13) para obter estimativas de $\tau$ e $\phi$.

3) Use

$$
\gamma_{0}=\sum_{j=1}^{p} \phi_{j} \gamma_{j}+\frac{1}{\tau}
$$

e 


$$
\gamma_{i}=\sum_{j=1}^{p} \phi_{j} \gamma_{j-i} \quad i>0
$$

para obter estimativas atualizadas de $\gamma_{p}$, substituindo as estimativas correntes de $\tau$ e $\phi$.

4) Repita os passos 2) e 3) até que $\gamma_{p}, \tau$ e $\phi$ estabilizem em pontos estacionários $\gamma_{p}^{*}$, $\tau^{*}$ e $\phi^{*}$.

Quanto à convergência deste processo iterativo, [Miller - 1995] afirma que embora não se possa provar formalmente a convergência, para um conjunto de dados suficientemente grande e bem modelado por um processo auto-regressivo estacionário, geralmente os pontos estacionários são alcançados rapidamente.

\subsection{Métodos Numéricos para Estimativas de Máxima Verossimilhança para Modelos MA(q)}

Veremos nesta seção dois métodos de estimação de máxima verossimilhança para a obtenção das estimativas dos parâmetros $\theta$ e $\sigma_{a}^{2}$ de um modelo MA(q), utilizando mínimos quadrados.

\subsubsection{Estimação de Máxima Verossimilhança Condicional}

Para o modelo MA(q) invertível

$$
\mathrm{z}_{t}=a_{t}-\theta_{1} a_{t-1}-\theta_{2} a_{t-2}-\ldots-\theta_{q} a_{t-q}
$$


supondo que os $a_{t}$ 's são normalmente distribuidos, a função densidade de probabilidade conjunta de $a_{1}, a_{2}, \ldots, a_{n}$ é dada por:

$$
p\left(a_{1}, a_{2}, \ldots, a_{n}\right)=\left(\frac{1}{2 \pi \sigma_{a}^{2}}\right)^{n / 2} \exp \left\{-\frac{1}{2 \sigma_{a}^{2}} \sum_{t=1}^{n} a_{t}^{2}\right\}
$$

Podemos reescrever (4.16) na seguinte forma:

$$
a_{t}=\mathrm{z}_{t}+\theta_{1} a_{t-1}+\theta_{2} a_{t-2}+\ldots+\theta_{q} a_{t-q}
$$

e, então, assumindo que q valores iniciais $a_{1-q}, a_{2-q}, \ldots, a_{0}$ são conhecidos, podemos obter a função de verossimilhança dos parâmetros. Suponhamos então que o vetor $a_{*}^{\prime}=\left(a_{1-q}\right.$, $\left.a_{2-q}, \ldots, a_{0}\right)$ seja dado. A função de verossimilhança condicional a esta escolha de $a_{*}^{\prime}$ é dada por:

$$
L_{*}\left(\boldsymbol{\theta}, \sigma_{a}^{2} \mid \mathbf{z}_{n}, a_{*}\right)=\left(\frac{1}{2 \pi \sigma_{a}^{2}}\right)^{n / 2} \exp \left\{-\frac{1}{2 \sigma_{a}^{2}} \sum_{t=1}^{n}\left(\mathrm{z}_{t}+\theta_{1} a_{t-1}+\ldots+\theta_{q} a_{t-q}\right)^{2}\right\}
$$

Aplicando o logaritmo e fazendo $\tau=\frac{1}{\sigma_{a}^{2}}$, a função log-verossimilhança condicional é:

$$
l_{*}\left(\theta, \tau \mid \mathbf{z}_{n}, a_{*}\right)=\frac{n}{2} \ln \left(\frac{\tau}{2 \pi}\right)-\frac{\tau}{2} \mathrm{~S}_{*}(\theta)
$$

onde

$$
\mathrm{S}_{*}(\boldsymbol{\theta})=\sum_{t=1}^{n} a_{t}^{2}\left(\boldsymbol{\theta} \mid \mathbf{z}_{n}, a_{*}\right)
$$

é a função soma de quadrados condicional.

Observando que a expressão (4.18) envolve os dados somente através de (4.19), segue que, para qualquer valor fixado $\tau$, o valor $\widehat{\theta}$ que minimiza $S_{*}(\theta)$ será também o valor que 
maximiza $l_{*}\left(\theta, \tau \mid \mathbf{z}_{n}, a_{*}\right)$, ou seja,$\widehat{\theta}$ será o estimador de máxima verossimilhança condicional para o modelo.

Uma maneira de especificar os valores do vetor $a_{*}$ é assumir $a_{1-q}=a_{2-q}=\ldots=$ $a_{0}=0$. A seguir, utilizando a expressão (4.17), podemos calcular $a_{t}, t=1, \ldots, n$ para vários valores de $\theta$ e conseqüentemente $S_{*}(\theta)$. Após obter a estimativa $\widehat{\theta}$, a estimativa $\widehat{\tau}$ de $\tau$ pode ser encontrada de

$$
\widehat{\tau}^{-1}=\frac{S_{*}(\widehat{\theta})}{d f}
$$

onde $d f$ é igual ao número de termos usado no somatório de $\mathrm{S}_{*}(\theta)$ menos o número de parâmetros estimados, ou seja, $d f=n-(\mathrm{q}+1)$.

\subsubsection{Estimação de Máxima Verossimilhança Incondicional}

$\mathrm{Na}$ seção 3.2 apresentamos na equação (3.20) a expressão da função de verossimilhança incondicional:

$$
L\left(\boldsymbol{\theta}, \tau \mid \mathbf{z}_{\mathrm{n}}\right)=\left(\frac{\tau}{2 \pi}\right)^{n / 2}|D|^{-1 / 2} \exp \left\{-\frac{\tau}{2} \sum_{t=1-q}^{n}\left[a_{t}\right]^{2}\right\}
$$

Aplicando o logaritmo, temos:

$$
l\left(\theta, \tau \mid \mathbf{z}_{n}\right)=\frac{n}{2} \ln \left(\frac{\tau}{2 \pi}\right)+f(\theta)-\frac{\tau}{2} \mathbf{S}(\theta)
$$

onde $f(\theta)$ é uma função de $\theta$ dada por $\ln |D|^{-1 / 2}$ e 


$$
S(\theta)=\sum_{t=1-q}^{n}\left[a_{t}\right]^{2}
$$

é a soma de quadrados incondicional, com

$$
\left[a_{t}\right]=E\left[a_{t} \mid \mathbf{z}_{n}, \theta\right]
$$

denotando a esperança condicional de $a_{t}$ dado $\mathbf{z}_{n}$ e $\theta$.

Para amostras de dados de tamanho moderado ou grande, o valor de $f(\theta)$ em (4.20) será pequeno. Conseqüentemente, estimativas mínimos quadrados de $\theta$ obtidas minimizando $S(\theta)$ serão boas aproximações para os estimadores de máxima verossimilhança.

O cálculo de $S(\theta)$ para um dado $\theta$, é feito gerando-se os $\left[a_{t}\right]$ 's recursivamente da expressão

$$
\left[a_{t}\right]=\left[\mathbf{z}_{t}\right]+\theta_{1}\left[a_{t-1}\right]+\theta_{2}\left[a_{t-2}\right]+\ldots+\theta_{q}\left[a_{t-q}\right]
$$

a qual é obtida tomando-se esperanças condicionais em (4.17). Entretanto, para inicializar este processo recursivo, é necessário o conhecimento dos valores iniciais $\left[z_{0}\right],\left[z_{-1}\right], \ldots,\left[z_{1-q}\right]$ afim de calcular $\left[a_{\mathrm{t}}\right]$ para $t<1$. Para gerar estes valores, utilizamos o procedimento sugerido por Box e Jenkins chamado "backforecasting" ou "previsão para o passado". Este procedimento é baseado no fato que o modelo (4.16) também pode ser escrito na forma "backward":

$$
\mathrm{z}_{t}=e_{t}-\theta_{1} e_{t+1}-\theta_{2} e_{t+2}-\ldots-\theta_{q} e_{t+q}
$$

ou

$$
\mathrm{z}_{t}=\left(1-\theta_{1} \mathrm{~F}-\theta_{2} \mathrm{~F}^{2}-\ldots-\theta_{q} \mathrm{~F}^{q}\right) e_{t}
$$


onde $e_{t}$ é um ruído branco com a mesma variância que $a_{t}$ e $\mathrm{F}$ é o operador translação para o futuro dado por $\mathrm{F}^{m} \mathrm{z}_{t}=\mathrm{z}_{t+m}$.

Reescrevendo (4.24) na forma

$$
e_{t}=\mathrm{z}_{t}+\theta_{1} e_{t+1}+\theta_{2} e_{t+2}+\ldots+\theta_{q} e_{t+q}
$$

e, tomando esperanças, vem:

$$
\left[e_{t}\right]=\left[\mathbf{z}_{t}\right]+\theta_{1}\left[e_{t+1}\right]+\theta_{2}\left[e_{t+2}\right]+\ldots+\theta_{q}\left[e_{t+q}\right]
$$

Então, a partir desta relação, assumindo que $\left[e_{t}\right]=0$ para $t=n+1, n+2, \ldots$, e usando o fato que $\left[\mathrm{z}_{t}\right]=\mathrm{z}_{t}$, para $t=1, \ldots, n$, calculamos:

$$
\begin{gathered}
{\left[e_{n}\right]=\left[\mathrm{z}_{n}\right]+\theta_{1}\left[e_{n+1}\right]+\ldots+\theta_{q}\left[e_{n+q}\right]} \\
{\left[e_{n-1}\right]=\left[\mathrm{z}_{n-1}\right]+\theta_{1}\left[e_{n}\right]+\ldots+\theta_{q}\left[e_{n-1+q}\right]} \\
\vdots \\
{\left[e_{0}\right]=\left[\mathrm{z}_{0}\right]+\theta_{1}\left[e_{1}\right]+\ldots+\theta_{q}\left[e_{q}\right]} \\
{\left[e_{-1}\right]=\left[\mathrm{z}_{-1}\right]+\theta_{1}\left[e_{0}\right]+\ldots+\theta_{q}\left[e_{q-1}\right]} \\
\vdots \\
{\left[e_{1-q}\right]=\left[\mathrm{z}_{1-q}\right]+\theta_{1}\left[e_{2-q}\right]+\ldots+\theta_{q}\left[e_{1}\right]}
\end{gathered}
$$

Como nas últimas q equações temos que $\left[e_{0}\right]=\left[e_{-1}\right]=\ldots=0$, pois $e_{0}, e_{-1}, \ldots$ são independentes de $\mathbf{z}_{n}$, podemos então tirar os valores de $\left[\mathrm{z}_{0}\right],\left[\mathrm{z}_{-1}\right], \ldots,\left[\mathrm{z}_{1-q}\right]$. Finalmente, usando a relação (4.23) geramos $\left[a_{t}\right], t=1-\mathrm{q}, 2-\mathrm{q}, \ldots, n$ levando em consideração o fato que $\left[a_{-q}\right]=\left[a_{-q-1}\right]=\ldots=0$, pois $a_{-q}, a_{-q-1}, \ldots$ são independentes de $\mathbf{z}_{n}$ num processo $\operatorname{MA}(q)$.

Realizamos este procedimento e calculamos $\mathrm{S}(\boldsymbol{\theta})$ para vários valores de $\boldsymbol{\theta}$. O valor $\widehat{\theta}$ que minimizar $\mathrm{S}(\boldsymbol{\theta})$ será então o estimador de máxima verossimilhança para o parâmetro $\boldsymbol{\theta}$. 
Após calcular a estimativa $\widehat{\boldsymbol{\theta}}$, a estimativa $\widehat{\tau}$ de $\tau$ pode ser encontrada de

$$
\widehat{\tau}^{-1}=\frac{S(\widehat{\theta})}{n}
$$

No capítulo 6, ilustraremos em exemplos o procedimento acima bem como o procedimento condicional da seção 4.2.1. 


\section{Capítulo 5 - Método de Simulação de Monte Carlo com Cadeia de Markov (MCMC) para Estimativas de Máxima Verossimilhança}

\subsection{Introdução}

Considerando a função de verossimilhança $L(\boldsymbol{\lambda}), \boldsymbol{\lambda} \epsilon \boldsymbol{\Lambda}$ (espaço de parâmetros), para os modelos $\mathrm{AR}(\mathrm{p})$ ou MA(q), podemos construir uma função densidade de probabilidade por:

$$
p(\boldsymbol{\lambda})=\frac{1}{\mathrm{c}} L(\boldsymbol{\lambda})
$$

onde

$$
\mathrm{c}=\int_{\lambda} L(\boldsymbol{\lambda}) d \boldsymbol{\lambda}
$$

Desta forma, o estimador de máxima verossimilhança $\widehat{\lambda}$ é a moda da densidade $p(\boldsymbol{\lambda})$.

A vantagem desta interpretação é que podemos calcular $\widehat{\lambda}$ usando algoritmos de simulação MCMC, sem necessidade do conhecimento da constante c. Esses algoritmos simulam no espaço de parâmetros uma cadeia de Markov cuja distribuição estacionária seja dada por $p$, de modo que, para uma iteração suficientemente alta da cadeia podemos considerar que os valores finais simulados representam amostras da densidade desejada.

Neste capítulo, apresentamos dois desses algoritmos que atualmente têm recebido considerável atenção. 


\subsection{Amostrador de Gibbs}

Seja $p(\lambda), \lambda=\left(\lambda_{1}, \lambda_{2}, \ldots, \lambda_{l}\right)$, uma função densidade conjunta da qual seja difícil gerar amostras diretamente. Porém, suponhamos que as densidades condicionais completas são conhecidas e possíveis de simular. O amostrador de Gibbs, [Casella e George - 1992], é um método iterativo que nos permite gerar amostras da densidade de interesse $p(\lambda)$ simulando cadeias de Markov através das condicionais.

$\mathrm{O}$ algoritmo simula amostras $\lambda_{i}^{(1)}, \lambda_{i}^{(2)}, \ldots, \lambda_{i}^{(s)}$ de cada parâmetro $\lambda_{i} \in \lambda$. A partir destas amostras podemos então, obter estimativas de máxima verossimilhança encontrando o vetor $\lambda^{*}$ que maximiza $p(\lambda)$. O algoritmo é baseado em sucessivas gerações das distribuições condicionais completas e é apresentado a seguir.

\section{Algoritmo:}

1) Faça $j=1$ e forneça um valor inicial arbitrário $\lambda^{(0)}=\left(\lambda_{1}^{(0)}, \ldots, \lambda_{1}^{(0)}\right)$.

2) Calcule urn novo valor $\lambda^{(j)}=\left(\lambda_{1}^{(j)}, \ldots, \lambda_{l}^{(j)}\right)$ a partir de $\lambda^{(j-1)}=\left(\lambda_{1}^{(j-1)}, \ldots, \lambda_{1}^{(j-1)}\right)$ através de sucessivas gerações de valores

$$
\begin{aligned}
\lambda_{1}^{(j)} & \sim p\left(\lambda_{1} \mid \lambda_{2}^{(j-1)}, \lambda_{3}^{(j-1)}, \ldots, \lambda_{l}^{(j-1)}\right) \\
\lambda_{2}^{(j)} & \sim p\left(\lambda_{2} \mid \lambda_{1}^{(j)}, \lambda_{3}^{(j-1)}, \ldots, \lambda_{l}^{(j-1)}\right) \\
& \vdots \\
\lambda_{l}^{(j)} & \sim p\left(\lambda_{l} \mid \lambda_{1}^{(j)}, \lambda_{2}^{(j)}, \ldots, \lambda_{l-1}^{(j)}\right)
\end{aligned}
$$

3) Faça $j \leftarrow j+1$ e repita 2) e 3) até a convergência.

A convergência deste algoritmo ocorre quando após um número finito de iterações temos que a amostra gerada é uma amostra da densidade conjunta $p(\boldsymbol{\lambda})$. 


\subsection{Algoritmo de Metropolis-Hastings}

Assim como o amostrador de Gibbs, o algoritmo de Metropolis-Hastings também é utilizado para gerar amostras de uma densidade $p(\lambda)$, mas no caso em que as densidades condicionais não têm forma padrão conhecida. Neste caso, podemos escrever $p(\lambda) \propto \Psi(\lambda) q(\lambda)$ onde $q(\lambda)$ é um núcleo com forma padrão conhecida e, portanto, é possível gerar amostras $\boldsymbol{\lambda}$ de $q(\lambda)$. Quando não é possível fatorar $p(\lambda)$, mesmo assim é possível escolher um núcleo $q(\lambda)$ para gerar novos valores. Métodos de escolha deste núcleo são dados em [Chib e Greenberg - 1995]. O algoritmo introduz uma probabilidade de aceitação do valor simulado de $q(\lambda)$, chamada "probabilidade de movimento", afim de garantir que esse valor represente uma amostra de $p(\lambda)$.

Apresentamos a seguir o algoritmo:

\section{Algoritmo:}

1) Faça $j=1$ e forneça um valor inicial arbitrário $\lambda^{(0)}=\left(\lambda_{1}^{(0)}, \ldots, \lambda_{l}^{(0)}\right)$.

2) Gere um novo valor $\beta$ a partir da densidade $q(\cdot)$.

3) Calcule a probabilidade de aceitação do novo valor gerado $\beta$ :

$$
\alpha\left(\lambda^{(j-1)}, \beta\right)=\min \left\{1, \frac{\Psi(\beta) q(\beta)}{\Psi\left(\lambda^{(j-1)}\right) q\left(\lambda^{(j-1)}\right)}\right\}, \Psi\left(\lambda^{(j-1)}\right) q\left(\lambda^{(j-1)}\right) \neq 0
$$

4) Gere uma variável aleatória uniforme $u \sim U(0,1)$.

5) Faça $\lambda^{(j)}=\left\{\begin{array}{l}\beta, \text { se } u \leq \alpha \\ \lambda^{(j-1)}, \text { caso contrário }\end{array}\right.$

6) Faça $j \leftarrow j+1$ e volte ao passo 2) até a convergência. 
Na prática, para os dois algoritmos apresentados, a formação da amostra $\lambda_{i}^{(1)}, \lambda_{i}^{(2)}, \ldots$, $\lambda_{i}^{(s)}$ de cada parâmetro de interesse $\lambda_{i} \in \lambda$, é feita tomando-se valores gerados após a convergência e espaçados de $\mathrm{k}$ iteraçōes (afim de obter amostras não correlacionadas).

Para identificar e monitorar a convergência, utilizamos aqui o critério de convergência proposto por [Gelman e Rubin -1992].

\subsection{Critério de Convergência de Gelman e Rubin}

O método proposto por Gelman e Rubin, consiste em utilizar paralelamente mais de uma cadeia com valores iniciais distintos e arbitrários e analisar a variância entre as médias de cada cadeia comparando com a média das variâncias dentro de cada cadeia. O método é formado pelos seguintes passos:

1- Simular $m \geq 2$ cadeias, iniciadas com valores distintos e arbitrários. Descartar uma parte inicial das iterações, para diminuir o efeito da dependência dos valores iniciais, e considerar as $n$ iteraçōes restantes.

2- Calcular para cada parâmetro escalar de interesse $\lambda$ :

$$
\begin{aligned}
& \frac{B}{n}=\sum_{i=1}^{m}\left(\lambda_{i .}-\lambda_{. .}\right)^{2} /(m-1) \quad: \text { variância entre as médias das } m \text { cadeias } \\
& W=\sum_{i=1}^{m} s_{i}^{2} / m: \text { média das variâncias dentro das } m \text { cadeias }
\end{aligned}
$$

onde $\lambda_{i}$ são as médias das $m$ sequiências, cada uma baseada em $n$ valores de $\lambda, \lambda$.. é a média destas médias e $s_{i}^{2}=\sum_{i=1}^{n}\left(\lambda_{i}-\dot{\lambda}_{i}\right)^{2} /(n-1)$.

3- Estimar a variância $\sigma^{2}$ por uma média ponderada de $B$ e $W$ da seguinte forma:

$$
\widehat{\sigma}^{2}=\frac{n-1}{n} W+\frac{1}{n} B
$$


Note que $\lambda$.. é uma média amostral usada como estimador de $\mu=\int \lambda p(\lambda) d \lambda$ e $\widehat{\sigma}^{2}$ é um estimador não viciado de $\sigma^{2}=\int(\lambda-\mu)^{2} p(\lambda) d \lambda$.

4- Sob a hipótese de que a distribuição de equilíbrio é $N\left(\lambda_{. .}, \widehat{\sigma}^{2}\right)$, adota-se uma aproximação desta distribuição por uma t-Student com parâmetro de locação $\lambda$.. e parâmetro de escala

$$
\sqrt{\widehat{V}}=\sqrt{\hat{\sigma}^{2}+\frac{B}{m n}}
$$

com grau de liberdade $d f$ :

$$
d f=\frac{2 \widehat{V}^{2}}{v \hat{a} r(\widehat{V})}
$$

onde

$$
\begin{aligned}
\operatorname{var}(\widehat{V})= & \left(\frac{n-1}{n}\right)^{2} \frac{1}{m} \operatorname{var}\left(\mathrm{s}_{i}^{2}\right)+\left(\frac{m+1}{m n}\right)^{2} \frac{2}{m-1} B^{2} \\
& +2 \frac{(m+1)(n-1)}{m n^{2}} \frac{n}{m}\left[\operatorname{cov} v\left(\mathrm{~s}_{i}^{2}, \lambda_{i}^{2}\right)-2 \lambda_{. .} \operatorname{cov}\left(\mathrm{s}_{i}^{2}, \lambda_{i}\right)\right]
\end{aligned}
$$

5- A convergência pode ser monitorada calculando-se o fator de redução de escala

$$
\sqrt{\widehat{R}}=\sqrt{\left(\frac{\widehat{V}}{W}\right) \frac{d f}{d f-2}}
$$

o qual decresce para 1 quando $\mathrm{n} \rightarrow \infty$.

6- Se $\sqrt{\widehat{R}}$ estiver próximo de 1 , a convergência é sugerida. Caso contrário mais simulações devem ser feitas.

Note que

$$
\frac{\widehat{V}}{W}=\frac{n-1}{n}+\frac{m+1}{m}\left(\frac{B / n}{W}\right)
$$


Portanto, quando a distribuição de equilíbrio é atingida, $W \rightarrow \sigma^{2}, \frac{B}{n} \rightarrow 0$ e $\frac{\widehat{V}}{W} \rightarrow 1$ quando $n \rightarrow \infty, \log 0 \sqrt{\widehat{R}} \approx 1$.

\subsection{Aplicação de Métodos MCMC para Processos AR(p)}

No capítulo 3, vimos na expressão (3.1.7) que a função de verossimilhança exata para processos $\operatorname{AR}(\mathrm{p})$ é da forma:

$$
L\left(\phi, \tau \mid \mathbf{z}_{n}\right)=\left(\frac{\tau}{2 \pi}\right)^{n / 2}\left|M_{p}^{(p)}\right|^{1 / 2} \exp \left\{-\frac{\tau}{2}\left[\mathbf{z}_{p} M_{p}^{(p)} \mathbf{z}_{p}+\sum_{t=p+1}^{n}\left(\mathrm{z}_{t}-\phi_{1} \mathbf{z}_{t-1}-\ldots-\phi_{p} \mathbf{z}_{t-p}\right)^{2}\right]\right\}
$$

As estimativas de máxima verossimilhança podem ser obtidas, aplicando-se o algoritmo de Metropolis-Hastings. Inicialmente, vamos reescrever a verossimilhança acima como o produto de duas funções. Para isso denotemos:

$$
Y=\left[\begin{array}{c}
z_{p+1} \\
z_{p+2} \\
\vdots \\
z_{n}
\end{array}\right]_{(n-p) \times 1}, \quad X=\left[\begin{array}{ccc}
z_{p} & \cdots & z_{1} \\
z_{p+1} & \cdots & z_{2} \\
\vdots & \vdots & \vdots \\
z_{n-1} & \cdots & z_{n-p}
\end{array}\right]_{(n-p) \times p} \quad \text { e } \quad \phi=\left[\begin{array}{c}
\phi_{1} \\
\phi_{2} \\
\vdots \\
\phi_{p}
\end{array}\right]_{p \times 1}
$$

Podemos então, escrever o somatório da expressão na forma

$$
\sum_{t=p+1}^{n}\left(\mathrm{z}_{t}-\phi_{1} \mathrm{z}_{t-1}-\ldots-\phi_{p} \mathrm{z}_{t-p}\right)^{2}=(Y-X \phi)^{\prime}(Y-X \phi)
$$

É possível mostrar que: 


$$
(Y-X \phi)^{\prime}(Y-X \phi)=(\phi-\widehat{\phi}) X^{\prime} X(\phi-\widehat{\phi})+(Y-\widehat{Y})^{\prime}(Y-\widehat{Y})
$$

onde

$$
\widehat{\phi}=\left(X^{\prime} X\right)^{-1} X^{\prime} Y
$$

e

$$
\widehat{Y}=X \widehat{\phi}=X\left(X^{\prime} X\right)^{-1} X^{\prime} Y
$$

O vetor $\widehat{\phi}$ é denominado o estimador de máxima verossimilhança quando usamos a função de verossimilhança "aproximada" - [Box, Jenkins e Reinsel - 1994].

Assim, podemos escrever a função de verossimilhança exata como

$$
L\left(\phi, \tau \mid \mathbf{z}_{n}\right)=\Psi_{1}(\phi, \tau) \Psi_{2}(\phi, \tau)
$$

definindo-se as funçōes

$$
\Psi_{1}(\phi, \tau)=\left(\frac{\tau}{2 \pi}\right)^{(n-1) / 2}\left|M_{p}^{(p)}\right|^{1 / 2} \exp \left\{-\frac{\tau}{2}\left[\mathbf{z}_{p} M_{p}^{(p)} \mathbf{z}_{p}+(Y-\widehat{Y})^{\prime}(Y-\widehat{Y})\right]\right\}
$$

$\mathrm{e}$

$$
\Psi_{2}(\phi, \tau)=\left(\frac{\tau}{2 \pi}\right)^{1 / 2} \exp \left[-\frac{\tau}{2}(\phi-\widehat{\phi}) X^{\prime} X(\phi-\widehat{\phi})\right]
$$

Portanto, $\Psi_{2}(\phi, \tau)$ é uma normal p-dimensional com média $\widehat{\phi}$ e precisão $\tau X^{\prime} X$.

Seja $p(\phi, \tau)=\Psi_{2}(\phi, \tau)$. Temos que

$$
\begin{aligned}
& p(\phi \mid \tau) \sim N\left(\widehat{\phi},\left(\tau X^{\prime} X\right)^{-1}\right) \\
& p(\tau \mid \phi) \sim \Gamma\left(\frac{3}{2}, \frac{1}{2}(\phi-\widehat{\phi}) X^{\prime} X(\phi-\widehat{\phi})\right)
\end{aligned}
$$

Então, podemos aplicar o algoritmo de Metropolis-Hastings utilizando as densidades acima para gerar os candidatos da amostra, sendo que a probabilidade de aceitação de cada candidato $\phi^{(i)} \sim p(\phi \mid \tau)$ é dada por 


$$
\alpha_{1}=\min \left\{1, \frac{\Psi_{1}\left(\phi^{(j)}, \tau\right)}{\Psi_{1}\left(\phi^{(j-1)}, \tau\right)}\right\}
$$

e a probabilidade de aceitação de $\tau^{(j)} \sim p(\tau \mid \phi)$ é dada por

$$
\alpha_{2}=\min \left\{1, \frac{\Psi_{1}\left(\phi, \tau^{(j)}\right)}{\Psi_{1}\left(\phi, \tau^{(j-1)}\right)}\right\}
$$

A aplicação do algoritmo para os casos particulares do $\operatorname{AR}(1)$ e $\operatorname{AR}(2)$, são ilustrados no próximo capítulo.

\subsection{Aplicação de Métodos MCMC para Processos MA(q)}

Vimos na seção 3.2 que para processos $\mathrm{MA}(\mathbf{q})$, a densidade conjunta de $\mathbf{z}_{n}$ e $\boldsymbol{a}_{*}$ é dada por:

$$
p\left(\mathbf{z}_{n}, a_{*} \mid \boldsymbol{\theta}, \tau\right)=\left(\frac{\tau}{2 \pi}\right)^{(n+q) / 2} \exp \left\{-\frac{\tau}{2}\left[\left(\mathbf{z}_{n}-\boldsymbol{F} a_{*}\right)^{\prime}{G^{\prime}}^{-1} \boldsymbol{G}^{-1}\left(\mathbf{z}_{n}-F a_{*}\right)+\boldsymbol{a}_{*}^{\prime} \boldsymbol{a}_{*}\right]\right\}
$$

que pode ser escrita na seguinte forma:

$$
\begin{aligned}
p\left(\mathbf{z}_{n}, a_{*} \mid \theta, \tau\right)= & \left(\frac{\tau}{2 \pi}\right)^{n / 2} \exp \left\{-\frac{\tau}{2}\left[\left(\mathbf{z}_{n}-\boldsymbol{F} \boldsymbol{a}_{*}\right)^{\prime}{\boldsymbol{G}^{\prime-1}}^{-1}\left(\mathbf{z}_{\mathbf{n}}-\boldsymbol{F} \boldsymbol{a}_{*}\right)\right]\right\} \\
& \cdot\left(\frac{\tau}{2 \pi}\right)^{q / 2} \exp \left\{-\frac{\tau}{2} \boldsymbol{a}_{*}^{\prime} \boldsymbol{a}_{*}\right\}
\end{aligned}
$$


A equação acima pode ser interpretada como

$$
p\left(\mathbf{z}_{n}, a_{*} \mid \boldsymbol{\theta}, \tau\right)=p\left(\mathbf{z}_{n} \mid a_{*}, \boldsymbol{\theta}, \tau\right) p\left(\boldsymbol{a}_{*} \mid \boldsymbol{\theta}, \tau\right)
$$

onde temos:

$$
p\left(\mathbf{z}_{n} \mid a_{*}, \boldsymbol{\theta}, \tau\right)=\left(\frac{\tau}{2 \pi}\right)^{n / 2} \exp \left\{-\frac{\tau}{2}\left[\left(\mathbf{z}_{n}-F a_{*}\right)^{\prime}{G^{\prime-1}}^{-1}\left(\mathbf{z}_{n}-F a_{*}\right)\right]\right\}
$$

e

$$
p\left(a_{*} \mid \theta, \tau\right)=\left(\frac{\tau}{2 \pi}\right)^{q / 2} \exp \left\{-\frac{\tau}{2} a_{*}^{\prime} a_{*}\right\}
$$

Supondo conhecido o valor de $a_{*}$, podemos estimar os parâmetros $\theta$ e $\tau$ usando a função de verossimilhança exata:

$$
L\left(\boldsymbol{\theta}, \tau \mid \mathbf{z}_{n}, \boldsymbol{a}_{*}\right)=p\left(\mathbf{z}_{n} \mid a_{*}, \boldsymbol{\theta}, \tau\right) p\left(\boldsymbol{a}_{*} \mid \boldsymbol{\theta}, \tau\right)
$$

ou seja,

$$
\begin{aligned}
L\left(\theta, \tau \mid \mathbf{z}_{n}, a_{*}\right)= & \left(\frac{\tau}{2 \pi}\right)^{n / 2} \exp \left\{-\frac{\tau}{2}\left[\left(\mathbf{z}_{n}-F a_{*}\right)^{\prime}{G^{\prime}}^{-1} G^{-1}\left(\mathbf{z}_{n}-F a_{*}\right)\right]\right\} \\
& \cdot\left(\frac{\tau}{2 \pi}\right)^{q / 2} \exp \left\{-\frac{\tau}{2} a_{*}^{\prime} a_{*}\right\}
\end{aligned}
$$

Observemos que:

$$
p\left(\theta \mid \tau, a_{*}, \mathbf{z}_{n}\right) \propto \tau^{n / 2} \exp \left\{-\frac{\tau}{2}\left[\left(\mathbf{z}_{n}-F a_{*}\right)^{\prime}{G^{\prime}}^{-1} G^{-1}\left(\mathbf{z}_{n}-F a_{*}\right)\right]\right\}
$$

e

$$
p\left(\tau \mid \boldsymbol{\theta}, a_{*}, \mathbf{z}_{n}\right) \propto \tau^{(\mathbf{n + q}) / 2} \exp \left\{-\frac{\tau}{2} \mathrm{~S}\left(\boldsymbol{\theta}, \boldsymbol{a}_{*}\right)\right\}
$$

onde 


$$
\mathrm{S}\left(\theta, a_{*}\right)=\left(\mathrm{z}_{\mathrm{n}}-F a_{*}\right)^{\prime}{G^{\prime}}^{-1} G^{-1}\left(\mathrm{z}_{n}-F a_{*}\right)+a_{*}^{\prime} a_{*}
$$

Então, utilizando os algoritmos de Gibbs e Metropolis-Hastings, implementamos o seguinte algoritmo:

1) Fazer $j=1$ e dar os valores iniciais $a_{*}^{(0)}, \tau^{(0)}$ e $\theta^{(0)}$;

2) Gerar:

$$
\begin{aligned}
& a_{*}^{(j)} \sim p\left(a_{*} \mid \theta^{(j-1)}, \tau^{(j-1)}\right) \\
& \tau^{(j)} \sim p\left(\tau \mid \theta^{(j-1)}, a_{*}^{(j)}, \mathbf{z}_{\mathbf{n}}\right) \\
& \theta^{(j)} \sim p\left(\theta \mid \tau^{(j)}, a_{*}^{(j)}, \mathbf{z}_{n}\right)
\end{aligned}
$$

3) Fazer $j \leftarrow j+1$ e repetir os passos 2) e 3) até convergência.

Note que:

$$
\begin{aligned}
a_{*} & \sim N\left(0, \tau^{-1}\right) \\
\tau & \sim \Gamma\left(\frac{n+q}{2}+1, \frac{\mathbf{s}\left(\theta, a_{*}\right)}{2}\right) \\
\theta & \sim p\left(\theta \mid \tau, \mathbf{z}_{n}, a_{*}\right) .
\end{aligned}
$$

Então, podemos utilizar o amostrador de Gibbs para gerar $a_{*} \mathrm{e} \tau$ e, no caso de $\theta$, como não é possível identificar uma distribuição conhecida, utilizamos o algoritmo de MetropolisHastings tomando como núcleo uma distribuição normal.

No próximo capítulo ilustraremos a aplicação conjunta do amostrador de Gibbs com o algoritmo Metropolis-Hastings para o caso MA(1) e para o caso MA(2). 


\section{Capítulo 6 - Aplicação}

\subsection{Aplicação aos Modelos AR(p)}

Ilustraremos aqui a aplicação dos métodos apresentados na seção 4.1 e do algoritmo Metropolis-Hastings para modelos AR(1) e AR(2). Para o caso AR(1), inicialmente apresentamos em detalhes a obtenção da função de verossimilhança exata.

\section{- Modelo AR(1):}

Vamos analisar o modelo auto-regressivo estacionário de ordem 1, ou AR(1),

$$
\mathbf{z}_{t}=\phi \mathbf{z}_{t-1}+a_{t}
$$

com $a_{t} \sim N\left(0, \tau^{-1}\right)$. A condição de estacionariedade do modelo é satisfeita quando para o parâmetro $\phi$ tivermos $-1<\phi<1$.

Suponhamos que o conjunto de observações $\mathbf{z}_{n}^{\prime}=\left(z_{1}, z_{2}, \ldots, z_{n}\right)$ seja gerado pelo modelo acima. Vamos primeiramente, construir a função de verossimilhaça para o processo.

Temos que a densidade de probabilidade conjunta de $\left(a_{2}, a_{3}, \ldots, a_{n}\right)$ é dada por

$$
p\left(a_{2}, a_{3}, \ldots, a_{n} \mid \mathrm{z}_{1}, \phi, \tau\right)=\left(\frac{\tau}{2 \pi}\right)^{(n-1) / 2} \exp \left\{-\frac{\tau}{2} \sum_{t=2}^{n} a_{t}^{2}\right\}
$$

Reescrevendo o modelo na forma

$$
\mathrm{z}_{t}-\phi \mathrm{z}_{t-1}=a_{t}
$$

e, fixando $\mathbf{z}_{p}^{\prime}=\left(\mathrm{z}_{1}, \mathrm{z}_{2}, \ldots, \mathrm{z}_{p}\right)$, podemos relacionar $\left(a_{2}, a_{3}, \ldots, a_{n}\right) \mathrm{e}\left(\mathrm{z}_{2}, \mathrm{z}_{3}, \ldots, \mathrm{z}_{n}\right)$ pela seguinte transformação:

$$
\begin{aligned}
& a_{2}=\mathrm{z}_{2}-\phi \mathrm{z}_{1} \\
& a_{3}=\mathrm{z}_{3}-\phi \mathrm{z}_{2} \\
& \vdots \\
& a_{n}=\mathrm{z}_{n}-\phi \mathrm{z}_{n-1}
\end{aligned}
$$


A transformação acima tem jacobiano unitário, o que implica que podemos escrever

$$
p\left(\mathrm{z}_{2}, \ldots, \mathrm{z}_{n} \mid \mathrm{z}_{1}, \phi, \tau\right)=\left(\frac{\tau}{2 \pi}\right)^{(\mathrm{n}-1) / 2} \exp \left\{-\frac{\tau}{2} \sum_{t=2}^{n}\left(\mathrm{z}_{t}-\phi \mathrm{z}_{t-1}\right)^{2}\right\}
$$

que é a função de verossimilhança aproximada (ou condicional). Podemos obter a função de verossimilhança exata, para os dados $\left(z_{1}, z_{2}, \ldots, z_{n}\right)$, através da seguinte equação:

$$
p\left(\mathrm{z}_{1}, \ldots, \mathrm{z}_{\mathrm{n}} \mid \phi, \tau\right)=p\left(\mathrm{z}_{2}, \ldots, \mathrm{z}_{\mathrm{n}} \mid \mathrm{z}_{1}, \phi, \tau\right) p\left(\mathrm{z}_{1} \mid \phi, \tau\right)
$$

Sendo

$$
p\left(\mathrm{z}_{1} \mid \phi, \tau\right)=\left(\frac{\tau}{2 \pi}\right)^{1 / 2}\left(1-\phi^{2}\right)^{1 / 2} \exp \left\{-\frac{\tau}{2}\left(1-\phi^{2}\right) \mathrm{z}_{1}^{2}\right\}
$$

então, segue que a verossimilhança exata é

$p\left(\mathbf{z}_{n} \mid \phi, \tau\right)=L\left(\phi, \tau \mid \mathbf{z}_{n}\right)=\left(\frac{\tau}{2 \pi}\right)^{n / 2}\left(1-\phi^{2}\right)^{1 / 2} \exp \left[-\frac{\tau}{2}\left\{\left(1-\phi^{2}\right) \mathrm{z}_{1}^{2}+\sum_{t=2}^{n}\left(\mathbf{z}_{t}-\phi \mathbf{z}_{t-1}\right)^{2}\right\}\right]$

e a log-verossimilhança

$$
l\left(\phi, \tau \mid \mathbf{z}_{n}\right)=\frac{n}{2} \ln (\tau)+\frac{1}{2} \ln \left(1-\phi^{2}\right)-\frac{\tau}{2}\left\{\left(1-\phi^{2}\right) \mathbf{z}_{1}^{2}+\sum_{t=2}^{n}\left(\mathbf{z}_{t}-\phi \mathbf{z}_{t-1}\right)^{2}\right\}
$$

A matriz $D$ definida na seção 3.1, para o modelo $A R(1)$ é dada por:

$$
D=\left[\begin{array}{cc}
D_{11} & -D_{12} \\
-D_{12} & D_{22}
\end{array}\right]
$$

onde

$$
\begin{aligned}
& D_{11}=\mathrm{z}_{1}^{2}+\mathrm{z}_{2}^{2}+\ldots+\mathrm{z}_{n}^{2} \\
& D_{12}=\mathrm{z}_{1} \mathrm{z}_{2}+\mathrm{z}_{2} \mathrm{z}_{3}+\ldots+\mathrm{z}_{n-1} \mathrm{z}_{n} \\
& D_{22}=\mathrm{z}_{2}+\mathrm{z}_{3}^{2}+\ldots+\mathrm{z}_{n-1}^{2}
\end{aligned}
$$

e, fazendo $S(\phi)=\phi_{u}^{\prime} D \phi_{u}$, onde $\phi_{u}^{\prime}=(1, \phi)$, obtemos

$$
S(\phi)=D_{11}-2 \phi D_{12}+\phi^{2} D_{22}
$$


Substituindo este valor na expressão

$$
l\left(\phi, \tau \mid \mathbf{z}_{n}\right)=\frac{n}{2} \ln (\tau)+\frac{1}{2} \ln \left(1-\phi^{2}\right)-\frac{\tau}{2} S(\phi)
$$

podemos então, aplicar os métodos numéricos apresentados na seção 4.1.

Nesses métodos, as estimativas para $\tau$ são obtidas resolvendo-se a expressão $\widehat{\tau}=n / S(\widehat{\phi})$. Para os métodos das seções 4.1.1 e 4.1.2, as estimativas para $\phi$ são dadas por $\widehat{\phi}=D_{12} / D_{22}$ e $\widehat{\phi}=(n-2) D_{12} /(n-1) D_{22}$, respectivamente. Para o método da seção 4.1.3, a estimativa de $\phi$ é obtida resolvendo iterativamente os sistemas

$$
\left\{\begin{array}{l}
D_{11}=\phi\left(D_{12}+\gamma_{1}\right)+n / \tau \\
D_{12}=\phi\left(D_{22}+\gamma_{0}\right)
\end{array}\right.
$$

e

$$
\left\{\begin{array}{l}
\gamma_{0}=\phi \gamma_{1}+1 / \tau \\
\gamma_{1}=\phi \gamma_{0}
\end{array}\right.
$$

até obter pontos estacionários.

Mostramos anteriormente no capítulo 3, que a expressão da função de verossimilhança exata para um modelo $\mathrm{AR}(1)$ é da forma

$$
L\left(\phi, \tau \mid \mathbf{z}_{n}\right)=\left(\frac{\tau}{2 \pi}\right)^{n / 2}\left(1-\phi^{2}\right)^{1 / 2} \exp \left[-\frac{\tau}{2}\left\{\left(1-\phi^{2}\right) \mathrm{z}_{1}^{2}+\sum_{t=2}^{n}\left(\mathrm{z}_{t}-\phi \mathrm{z}_{t-1}\right)^{2}\right\}\right]
$$

Para a aplicação do algoritmo de Metropolis-Hastings, vimos na seção 5.5 que devemos reescrever esta função como

$$
\begin{aligned}
L\left(\phi, \tau \mid \mathbf{z}_{n}\right)= & \left(\frac{\tau}{2 \pi}\right)^{n / 2}\left(1-\phi^{2}\right)^{1 / 2} \exp \left[-\frac{\tau}{2}\left(1-\phi^{2}\right) \mathrm{z}_{1}^{2}\right] . \\
& \cdot \exp \left[-\frac{\tau}{2}\left\{(\phi-\widehat{\phi}) X^{\prime} X(\phi-\widehat{\phi})+(Y-\widehat{Y})^{\prime}(Y-\widehat{Y})\right\}\right]
\end{aligned}
$$

onde

$$
Y=\left[\begin{array}{c}
\mathrm{z}_{2} \\
\mathrm{z}_{3} \\
\vdots \\
\mathrm{z}_{n}
\end{array}\right], \quad X=\left[\begin{array}{c}
\mathrm{z}_{1} \\
\mathrm{z}_{2} \\
\vdots \\
\mathrm{z}_{n-1}
\end{array}\right] \text {, }
$$




$$
\widehat{\phi}=\left(X^{\prime} X\right)^{-1} X^{\prime} Y
$$

e

$$
\widehat{Y}=X \widehat{\phi}=X\left(X^{\prime} X\right)^{-1} X^{\prime} Y
$$

Agora, definindo as funções

$$
\Psi_{1}(\phi, \tau)=\left(\frac{\tau}{2 \pi}\right)^{(n-1) / 2}\left(1-\phi^{2}\right)^{1 / 2} \exp \left[-\frac{\tau}{2}\left\{\left(1-\phi^{2}\right) z_{1}^{2}+(Y-\widehat{Y})^{\prime}(Y-\widehat{Y})\right\}\right]
$$

e

$$
\Psi_{2}(\phi, \tau)=\left(\frac{\tau}{2 \pi}\right)^{1 / 2} \exp \left[-\frac{\tau}{2}(\phi-\widehat{\phi}) X^{\prime} X(\phi-\widehat{\phi})\right]
$$

podemos escrever

$$
L\left(\phi, \tau \mid \mathbf{z}_{n}\right)=\Psi_{1}(\phi, \tau) \Psi_{2}(\phi, \tau)
$$

Seja $p(\phi, \tau)=\Psi_{2}(\phi, \tau)$. Então, a aplicação do algoritmo pode ser feita simulando-se valores das densidades

$$
\begin{aligned}
& p(\phi \mid \tau) \sim N\left(\widehat{\phi},\left(\tau X^{\prime} X\right)^{-1}\right) \\
& p(\tau \mid \phi) \sim \Gamma\left(\frac{3}{2}, \frac{1}{2}(\phi-\widehat{\phi}) X^{\prime} X(\phi-\widehat{\phi})\right)
\end{aligned}
$$

e usando a probabilidade de aceitação para $\phi^{(j)}$ dado $\tau^{(j-1)}$

$$
\alpha_{1}=\min \left\{1, \frac{\Psi_{1}\left(\phi^{(j)}, \tau\right)}{\Psi_{1}\left(\phi^{(j-1)}, \tau\right)}\right\}
$$

para cada valor simulado $\phi^{(j)}$, e a probabilidade de aceitação de $\tau^{(j)}$

$$
\alpha_{2}=\min \left\{1, \frac{\Psi_{1}\left(\phi, \tau^{(j)}\right)}{\Psi_{1}\left(\phi, \tau^{(j-1)}\right)}\right\}
$$


Como ilustração, vamos apresentar a aplicação dos resultados acima para a série de dados reais formada pelas vazões médias mensais do reservatório de Sobradinho durante 48 anos, no período de janeiro de 1931 a dezembro de 1978. O gráfico da série é dado pela figura a seguir.

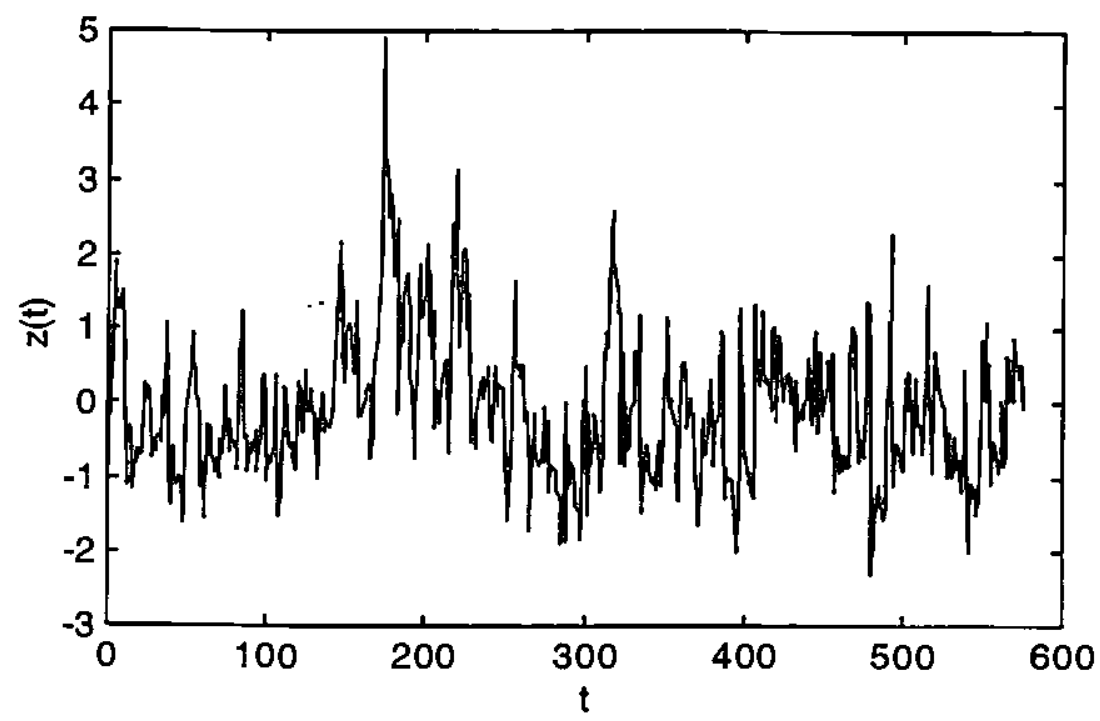

fig.1 - Gráfico da série real Sobradinho com 576 observaçðes.

As estimativas obtidas por cada um dos métodos são dados na seguinte tabela:

Tabela 1 - Valores ajustados para a série Sobradinho.

\begin{tabular}{|l|l|l|}
\hline & & \\
\hline Estimat. Mínimos Quadrados & 2.53854 & 0.74961 \\
\hline Aproximação das E.M.V. & 2.53853 & 0.74831 \\
\hline ARMLE & 2.53853 & 0.74832 \\
\hline Metropolis-Hastings & 2.52236 & 0.75113 \\
\hline
\end{tabular}

Observamos que para os métodos numéricos, os resultados foram bastante próximos e que não houve muita diferença entre os valores obtidos destes métodos com o método MCMC.

A seguir, mostramos o gráfico gerado através da expressão da função de verossimilhança exata para um modelo AR(1), calculada a partir dos dados $\operatorname{com} \tau$ variando no intervalo $[1.8,3.2]$ e $\phi$ no intervalo $[0.55,0.95]$. 


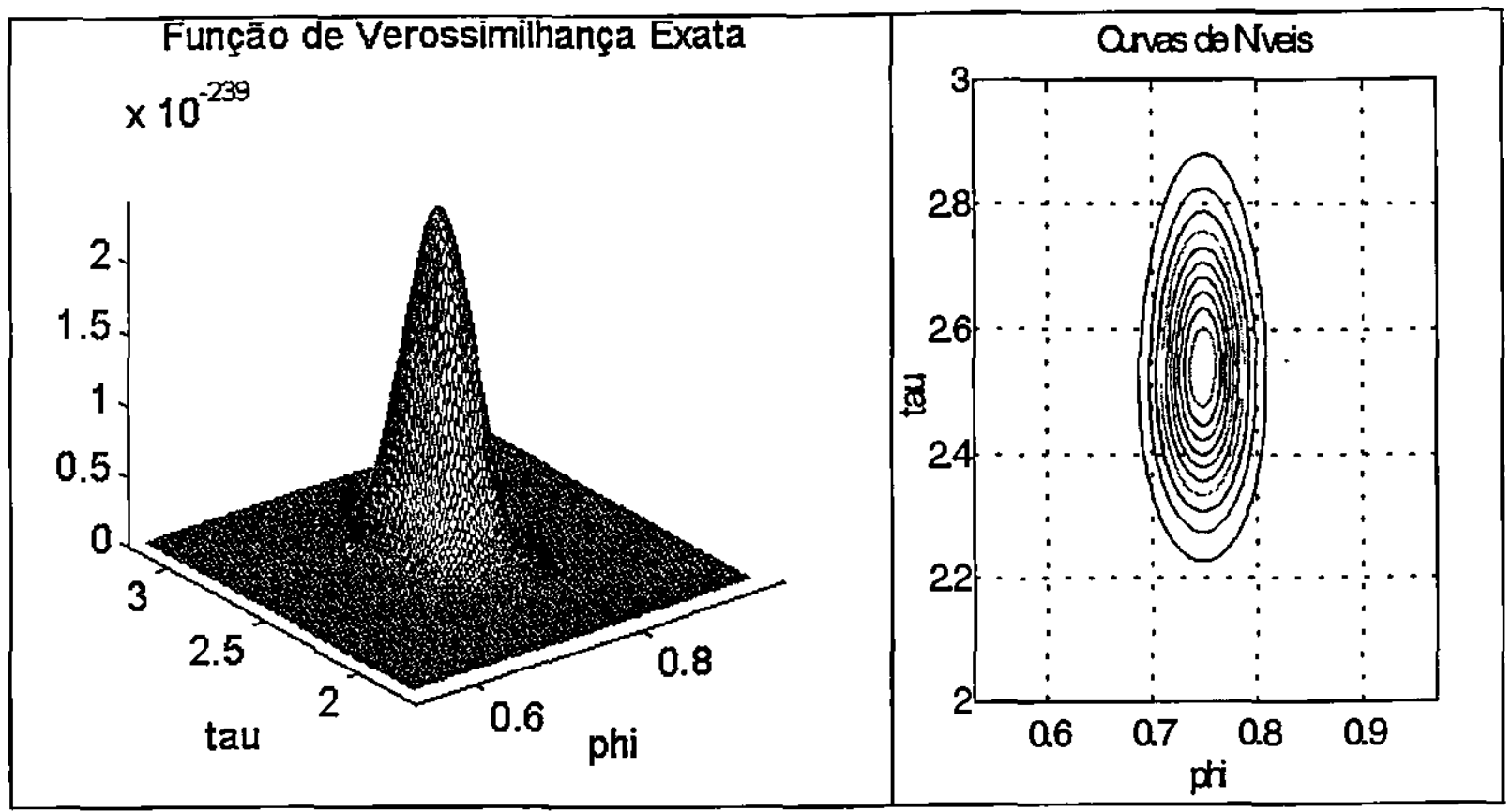

fig. 2 - Gráfico da função de verossimilhança e curvas de níveis para a série real Sobradinho.

A partir da estimativa $\widehat{\phi}=0.75113$ obtida por Metropolis-Hastings, calculamos previsões de 1 passo à frente (vide apêndice) para 36 dados da série, referentes ao período de janeiro de 1979 a dezembro de 1981. As previsões calculadas e os dados reais são mostrados no gráfico seguinte.

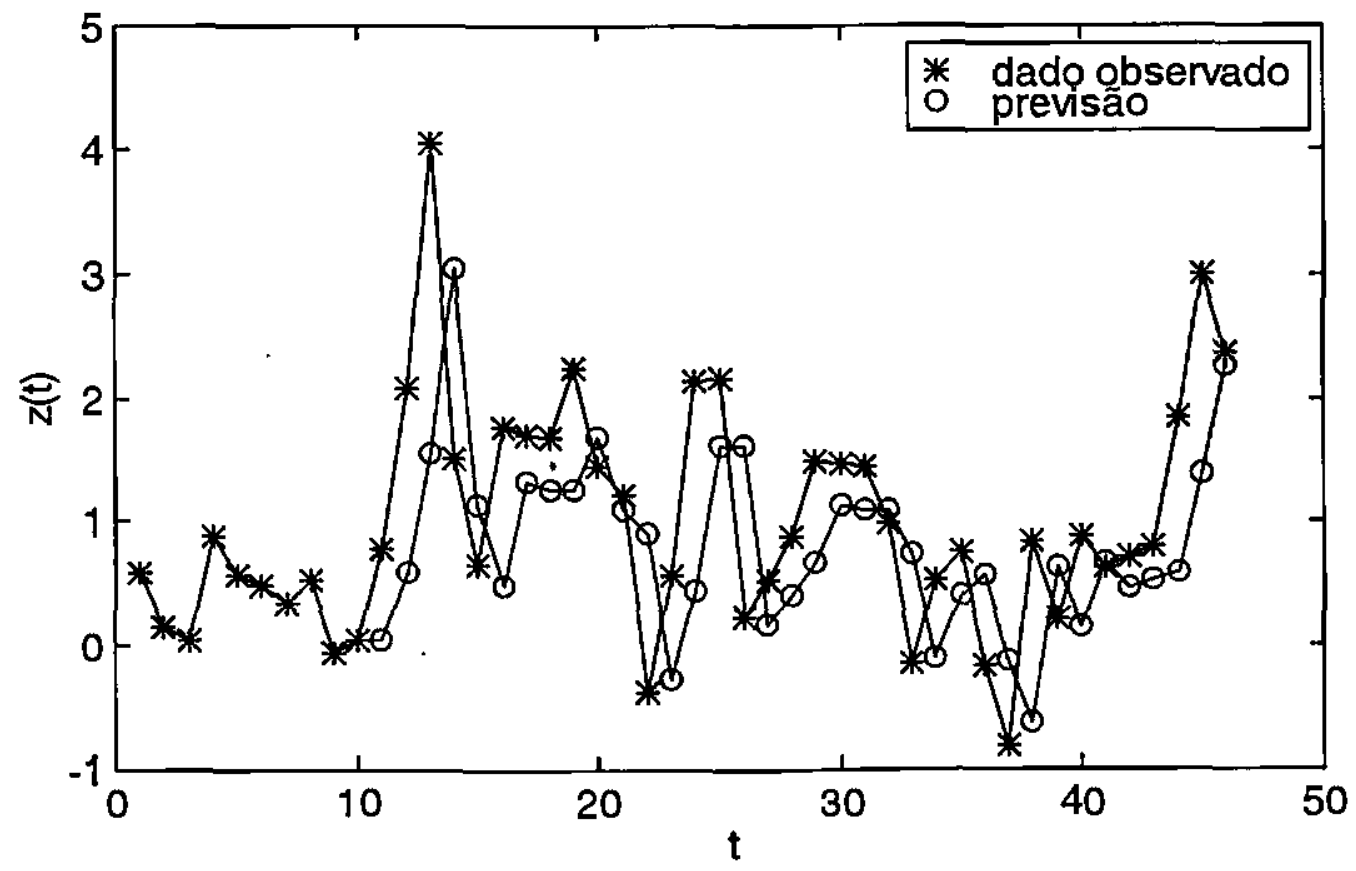

fig. 3 - Previsão 1 passo à frente para 36 meses da série Sobradinho. 
No caso do algoritmo de Metropolis-Hastings, para cada parâmetro foram simuladas 2 cadeias com 22500 iterações cada. Para a amostra final, foi descartada $30 \%$ da parte inicial de cada cadeia, e tomados valores espaçados de 30 iterações (devido à alta correlação entre os valores gerados), fazendo um total de 1050 amostras para cada um dos parâmetros de interesse.

Na tabela 2, apresentamos um resumo dos resultados obtidos pelo algoritmo.

Tabela 2 - Metropolis-Hastings para a série Sobradinho.

\begin{tabular}{|l|c|c|}
\hline \multicolumn{1}{|c|}{} & 2.52582 & 0.74930 \\
\hline Média & 2.52236 & 0.75113 \\
\hline Moda (EMV) & 2.51964 & 0.74966 \\
\hline$\sqrt{\widehat{\mathrm{R}}}$ - (critério de converg.) & 1.00319 & 0.99923 \\
\hline
\end{tabular}

Usamos o critério de Gelman e Rubin para verificar a convergência e neste caso $\sqrt{\widehat{\mathrm{R}}}<1.01$.

Nas próximas figuras, apresentamos os histogramas construídos com as amostras selecionadas para cada parâmetro e, em seguida, os gráficos mostrando a convergência das duas cadeias simuladas .

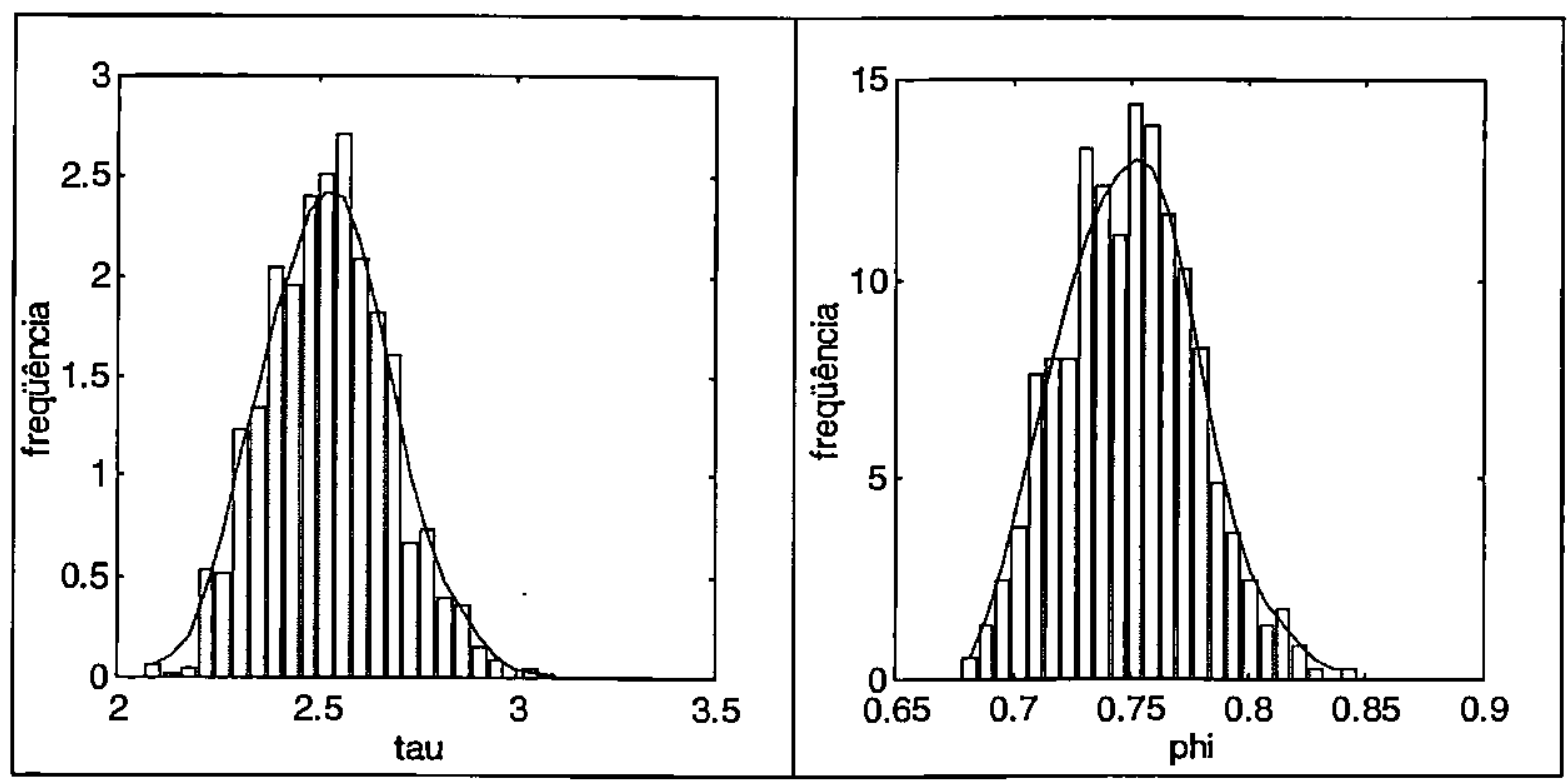

fig. 4 - Histogramas das distribuições aproximadas de $\tau$ e $\phi$. 

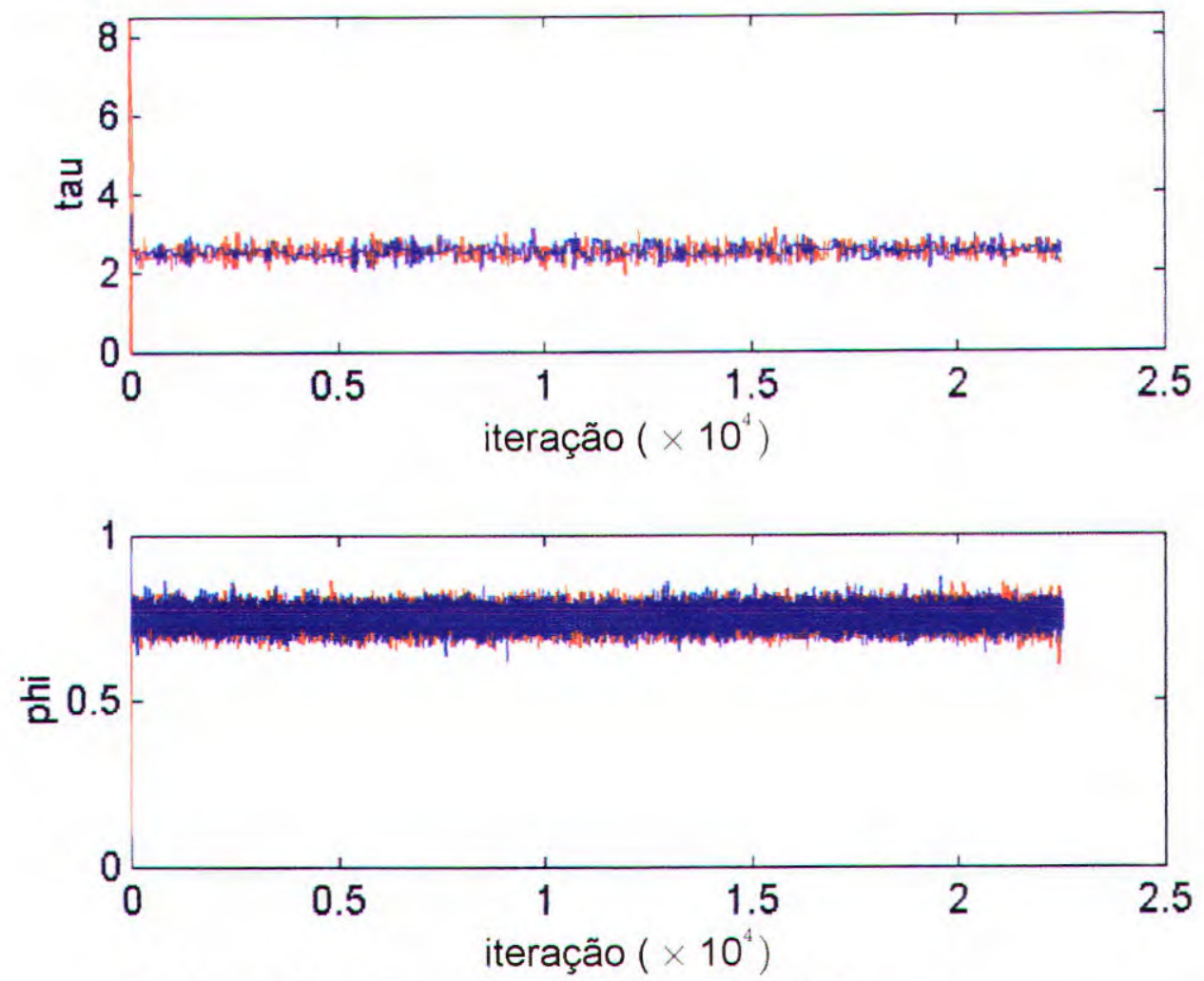

fig. 5 - Gráficos com todos os valores simulados para $\tau$ e $\phi$.

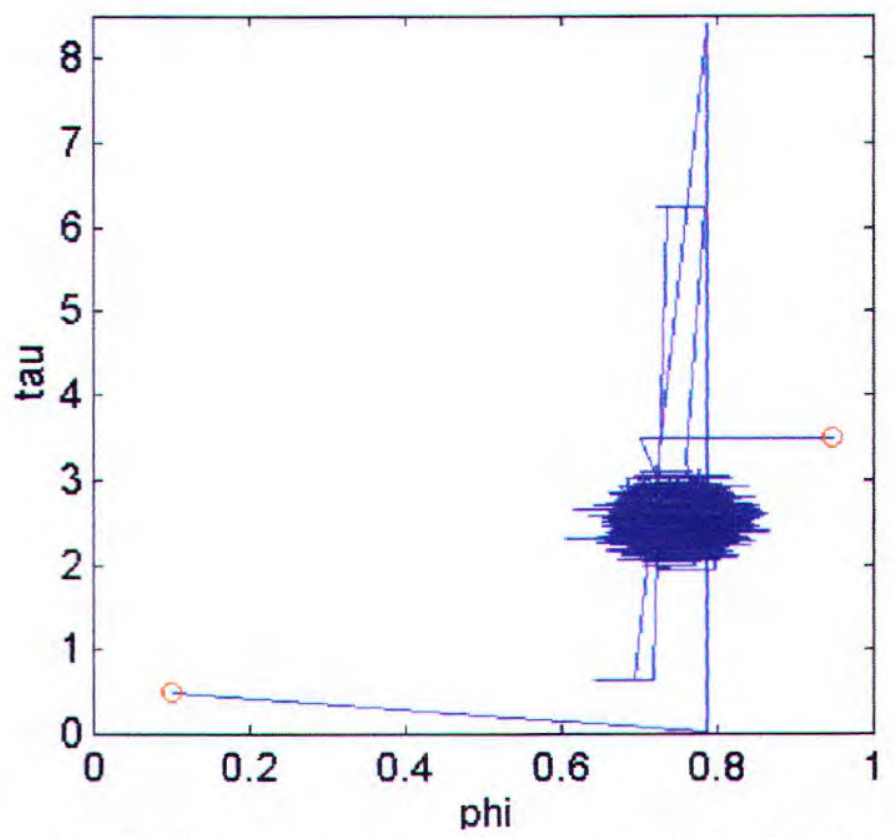

fig. 6 - Gráfico mostrando a convergência das duas cadeias geradas, com seus repectivos pontos de partida. 


\section{- Modelo AR(2):}

Vamos agora analisar o modelo auto-regressivo estacionário de ordem 2, ou AR(2),

$$
\mathrm{z}_{t}=\phi_{1} \mathrm{z}_{t-1}+\phi_{2} \mathrm{z}_{t-2}+a_{t}
$$

onde $a_{t} \sim N\left(0, \tau^{-1}\right)$. Para garantir a estacionariedade, pode ser mostrado que $\phi_{1}$ e $\phi_{2}$ devem satisfazer as seguintes condições: $\phi_{2}+\phi_{1}<1, \phi_{2}-\phi_{1}<1$ e $-1<\phi_{2}<1$. Suponhamos que o conjunto de observações da série $\mathbf{z}_{n}^{\prime}=\left(z_{1}, z_{2}, \ldots, z_{n}\right)$ seja gerado pelo modelo acima.

No capítulo 3, vimos que a função de verossimilhança exata para este modelo é:

$$
\begin{aligned}
L\left(\phi, \tau \mid \mathbf{z}_{n}\right)= & \left(\frac{\tau}{2 \pi}\right)^{n / 2}\left[\left(1+\phi_{2}\right)^{2}\left\{\left(1-\phi_{2}\right)^{2}-\phi_{1}^{2}\right\}\right]^{\frac{1}{2}} \\
& \exp \left[-\frac{\tau}{2}\left\{\left(1-\phi_{2}^{2}\right)\left(\mathrm{z}_{1}^{2}+\mathrm{z}_{2}^{2}\right)-2 \phi_{1}\left(1+\phi_{2}\right) \mathrm{z}_{1} \mathrm{z}_{2}\right\}\right] \\
& \exp \left[-\frac{\tau}{2} \sum_{t=3}^{n}\left(\mathrm{z}_{t}-\phi_{1} \mathrm{z}_{t-1}-\phi_{2} \mathrm{z}_{t-2}\right)^{2}\right]
\end{aligned}
$$

Temos que a matriz $D$, para o modelo é dada por:

$$
D=\left[\begin{array}{ccc}
D_{11} & -D_{12} & -D_{13} \\
-D_{12} & D_{22} & D_{23} \\
-D_{13} & D_{23} & D_{33}
\end{array}\right]
$$

onde

$$
\begin{aligned}
& D_{11}=\mathrm{z}_{1}^{2}+\mathrm{z}_{2}^{2}+\ldots+\mathrm{z}_{n}^{2} \\
& D_{12}=\mathrm{z}_{1} \mathrm{z}_{2}+\mathrm{z}_{2} \mathrm{z}_{3}+\ldots+\mathrm{z}_{n-1} \mathrm{z}_{n} \\
& D_{13}=\mathrm{z}_{1} \mathrm{z}_{3}+\mathrm{z}_{2} \mathrm{z}_{4}+\ldots+\mathrm{z}_{n-2} \mathrm{z}_{n} \\
& D_{22}=\mathrm{z}_{2}^{2}+\mathrm{z}_{3}^{2}+\ldots+\mathrm{z}_{n-1}^{2} \\
& D_{23}=\mathrm{z}_{2} \mathrm{z}_{3}+\mathrm{z}_{3} \mathrm{z}_{4}+\ldots+\mathrm{z}_{n-2} \mathrm{z}_{n-1} \\
& D_{33}=\mathrm{z}_{3}^{2}+\mathrm{z}_{4}^{2}+\ldots+\mathrm{z}_{n-2}^{2}
\end{aligned}
$$

e, fazendo $S(\phi)=\phi_{u}^{\prime} D \phi_{u}$, onde $\phi_{u}^{\prime}=\left(1, \phi_{1}, \phi_{2}\right)$, obtemos

$$
S(\phi)=D_{11}-2 \phi_{1} D_{12}-2 \phi_{2} D_{13}+2 \phi_{1} \phi_{2} D_{23}+\phi_{1}^{2} D_{22}+\phi_{2}^{2} D_{33}
$$


Substituindo este valor na expressão

$$
l\left(\phi, \tau \mid \mathbf{z}_{n}\right)=\frac{n}{2} \ln (\tau)+\frac{1}{2} \ln \left[\left(1+\phi_{2}\right)^{2}\left\{\left(1-\phi_{2}\right)^{2}-\phi_{1}^{2}\right\}\right]-\frac{\tau}{2} S(\phi)
$$

podemos aplicar os três métodos apresentados no capítulo 4.

As estimativas para $\tau$ são obtidas resolvendo-se a expressão $\widehat{\tau}=n / S(\widehat{\phi})$. Para os métodos das seções 4.1.1 e 4.1.2, as estimativas para $\phi_{1}$ e $\phi_{2}$ são dadas por

$$
\left[\begin{array}{l}
\widehat{\phi}_{1} \\
\widehat{\phi}_{2}
\end{array}\right]=\frac{1}{D_{22} D_{33}-D_{23}^{2}}\left[\begin{array}{cc}
D_{33} & -D_{23} \\
-D_{23} & D_{22}
\end{array}\right] \cdot\left[\begin{array}{c}
D_{12} \\
D_{13}
\end{array}\right]
$$

e

$$
\left[\begin{array}{l}
\widehat{\phi}_{1} \\
\widehat{\phi}_{2}
\end{array}\right]=\frac{1}{D_{22}^{*} D_{33}^{*}-\left(D_{23}^{*}\right)^{2}}\left[\begin{array}{cc}
D_{33}^{*} & -D_{23}^{*} \\
-D_{23}^{*} & D_{22}^{*}
\end{array}\right] \cdot\left[\begin{array}{c}
D_{12}^{*} \\
D_{13}^{*}
\end{array}\right]
$$

respectivamente.

Para o método da seção 4.1.3, a estimativa de $\phi$ é obtida resolvendo-se iterativamente os sistemas

$$
\left\{\begin{array}{l}
D_{11}=\phi_{1}\left(D_{12}+\gamma_{1}\right)+\phi_{2}\left(D_{13}+2 \gamma_{2}\right)+n / \tau \\
D_{21}=\phi_{1}\left(D_{22}+\gamma_{0}\right)+\phi_{2}\left(D_{23}+2 \gamma_{1}\right) \\
D_{31}=\phi_{1}\left(D_{32}+\gamma_{1}\right)+\phi_{2}\left(D_{33}+2 \gamma_{0}\right)
\end{array}\right.
$$

e

$$
\left\{\begin{array}{l}
\gamma_{0}=\phi_{1} \gamma_{1}+\phi_{2} \gamma_{2}+1 / \tau \\
\gamma_{1}=\phi_{1} \gamma_{0}+\phi_{2} \gamma_{1} \\
\gamma_{2}=\phi_{1} \gamma_{1}+\phi_{2} \gamma_{0}
\end{array}\right.
$$

até obter pontos estacionários.

Para a aplicação do algoritmo de Metropolis-Hastings, vamos reescrever a função de verossimilhança exata $L\left(\phi, \tau \mid \mathbf{z}_{n}\right)$, dada no início do exemplo, como:

$$
\begin{aligned}
L\left(\phi, \tau \mid \mathbf{z}_{n}\right)= & \left(\frac{\tau}{2 \pi}\right)^{\mathrm{n} / 2}\left[\left(1+\phi_{2}\right)^{2}\left\{\left(1-\phi_{2}\right)^{2}-\phi_{1}^{2}\right\}\right]^{\frac{1}{2}} . \\
& \exp \left[-\frac{\tau}{2}\left\{\left(1-\phi_{2}^{2}\right)\left(\mathrm{z}_{1}^{2}+\mathrm{z}_{2}^{2}\right)-2 \phi_{1}\left(1+\phi_{2}\right) \mathrm{z}_{1} \mathrm{z}_{2}\right\}\right] . \\
& \exp \left[-\frac{\tau}{2}\left\{(\boldsymbol{\phi}-\widehat{\phi})^{\prime} X^{\prime} X(\boldsymbol{\phi}-\widehat{\phi})+(Y-\widehat{Y})^{\prime}(Y-\widehat{Y})\right\}\right]
\end{aligned}
$$


onde

$$
Y=\left[\begin{array}{c}
z_{3} \\
z_{4} \\
\vdots \\
z_{n}
\end{array}\right], \quad X=\left[\begin{array}{cc}
z_{2} & z_{1} \\
z_{3} & z_{2} \\
\vdots & \vdots \\
z_{n-1} & z_{n-2}
\end{array}\right] \quad \text { e } \quad \phi=\left[\begin{array}{l}
\phi_{1} \\
\phi_{2}
\end{array}\right]
$$

Agora, definindo as funçōes

$$
\begin{aligned}
\Psi_{1}(\phi, \tau)= & \left(\frac{\tau}{2 \pi}\right)^{(\mathrm{n}-1) / 2}\left[\left(1+\phi_{2}\right)^{2}\left\{\left(1-\phi_{2}\right)^{2}-\phi_{1}^{2}\right\}\right]^{\frac{1}{2}} \\
& \exp \left[-\frac{\tau}{2}\left\{\left(1-\phi_{2}^{2}\right)\left(\mathrm{z}_{1}^{2}+\mathrm{z}_{2}^{2}\right)-2 \phi_{1}\left(1+\phi_{2}\right) \mathrm{z}_{1} \mathrm{z}_{2}\right\}\right] \\
& \exp \left[-\frac{\tau}{2}(Y-\widehat{Y})^{\prime}(Y-\widehat{Y})\right]
\end{aligned}
$$

e

$$
\Psi_{2}(\phi, \tau)=\left(\frac{\tau}{2 \pi}\right)^{1 / 2} \exp \left[-\frac{\tau}{2}(\phi-\widehat{\phi})^{\prime} X^{\prime} X(\phi-\widehat{\phi})\right]
$$

a função de verossimilhança fica

$$
L\left(\phi, \tau \mid \mathbf{z}_{n}\right)=\Psi_{1}(\phi, \tau) \Psi_{2}(\phi, r)
$$

Seja $p(\phi, \tau)=\Psi_{2}(\phi, \tau)$. Então, simulamos amostras através das densidades

$$
p(\phi \mid \tau) \sim N\left(\widehat{\phi},\left(\tau X^{\prime} X\right)^{-1}\right)
$$

e

$$
p(\tau \mid \phi) \sim \Gamma\left(\frac{3}{2}, \frac{1}{2}(\phi-\widehat{\phi})^{\prime} X^{\prime} X(\phi-\widehat{\phi})\right)
$$

e usamos como probabilidade de aceitação de $\phi^{(i)}$

$$
\alpha_{1}=\min \left\{\mathrm{I}, \frac{\Psi_{1}\left(\phi^{(\mathrm{j})}, \tau\right)}{\Psi_{1}\left(\phi^{(\mathrm{j}-1)}, \tau\right)}\right\}
$$

e como probabilidade de aceitação de $\tau^{(0)}$

$$
\alpha_{2}=\min \left\{1, \frac{\Psi_{1}\left(\phi, \tau^{(j)}\right)}{\Psi_{1}\left(\phi, \tau^{(j-1)}\right)}\right\}
$$


Apresentamos a seguir, a aplicação dos resultados acima para uma série simulada pelo modelo $\operatorname{AR}(2) z_{t}=0.5 z_{t-1}-0.7 z_{t-2}+a_{t}$ com 150 observações e também para uma série de dados reais formada pelas vazōes médias mensais do reservatório de Furnas durante 48 anos no período de janeiro de 1931 a dezembro de 1978. Na implementação do algoritmo de Metropolis-Hastings, para cada parâmetro foram simuladas 2 cadeias com 20000 iterações cada e descartados $30 \%$ de cada uma. Também, para a série simulada foram tomados valores espaçados de 15 iterações totalizando 1868 amostras e para a série Furnas valores espaçados de 20 iterações, totalizando uma amostra de tamanho1400. Este critério de seleção de valores espaçados foi baseado na correlação dos valores simulados.

Série simulada $\mathrm{z}_{\mathrm{t}}=0.5 \mathrm{z}_{\mathrm{t}-1}-0.7 \mathrm{z}_{\mathrm{t}-2}+a_{\mathrm{t}}$ :

Mostramos na figura abaixo o gráfico da série simulada e, a seguir, na tabela 3 os valores estimados para os parâmetros utilizando-se cada método.

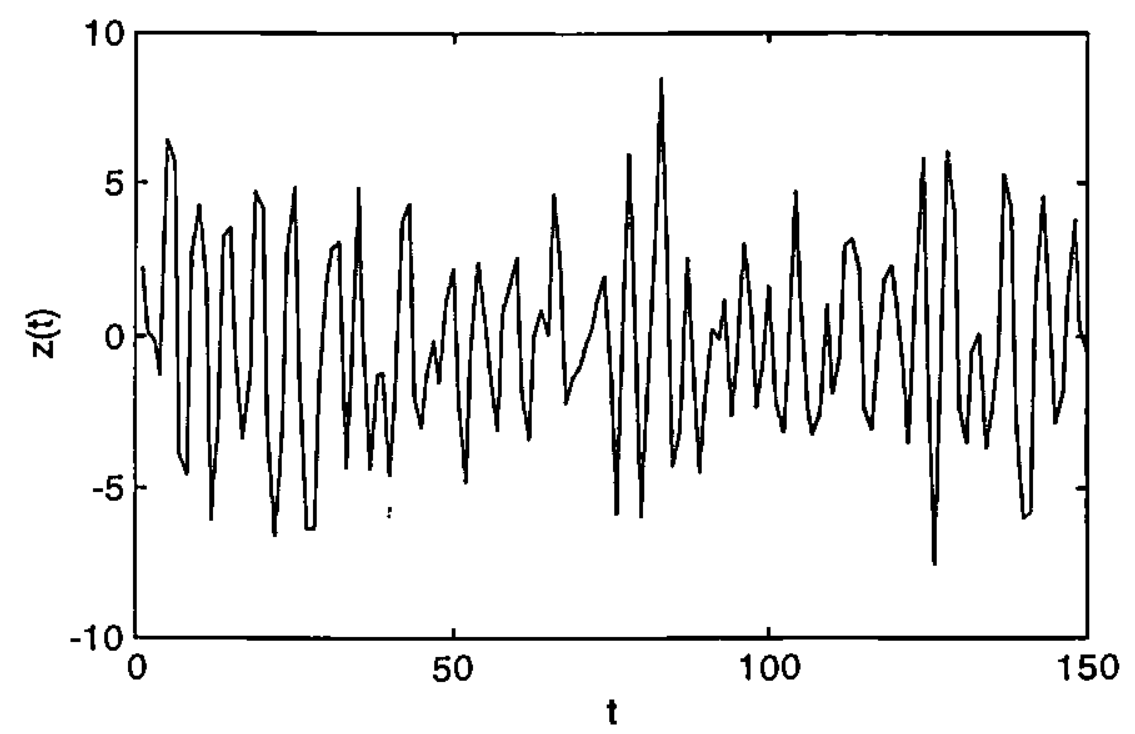

fig. 7 - Gráfico da série $z_{t}=0.5 z_{t-1}-0.7 z_{t-2}+a_{t}$ 
Tabela 3 - Valores ajustados para o modelo: $z_{t}=0.5 z_{t-1}-0.7 z_{t-2}+a_{t}$, onde $a_{\mathrm{t}} \sim N(0,4), \tau=0.25$.

\begin{tabular}{|l|l|l|l|}
\hline \multicolumn{1}{|c|}{} & & & \\
\hline Estimat. Mínimos Quadrados & 0.23736 & 0.48783 & -0.73973 \\
\hline Aproximação das E.M.V. & 0.23731 & 0.48454 & -0.72974 \\
\hline ARMLE & 0.23731 & 0.48467 & -0.73021 \\
\hline Metropolis-Hastings & 0.23668 & 0.48191 & -0.73807 \\
\hline
\end{tabular}

Analisando os resultados, concluímos que as estimativas de cada método estão muito próximas. Apresentamos abaixo o gráfico da função de verossimilhança exata da série, gerado $\operatorname{com} \tau=0.237, \phi_{1}$ variando no intervalo $[0.2,0.75]$ e $\phi_{2}$ variando no intervalo $[-0.95,-0.5]$.

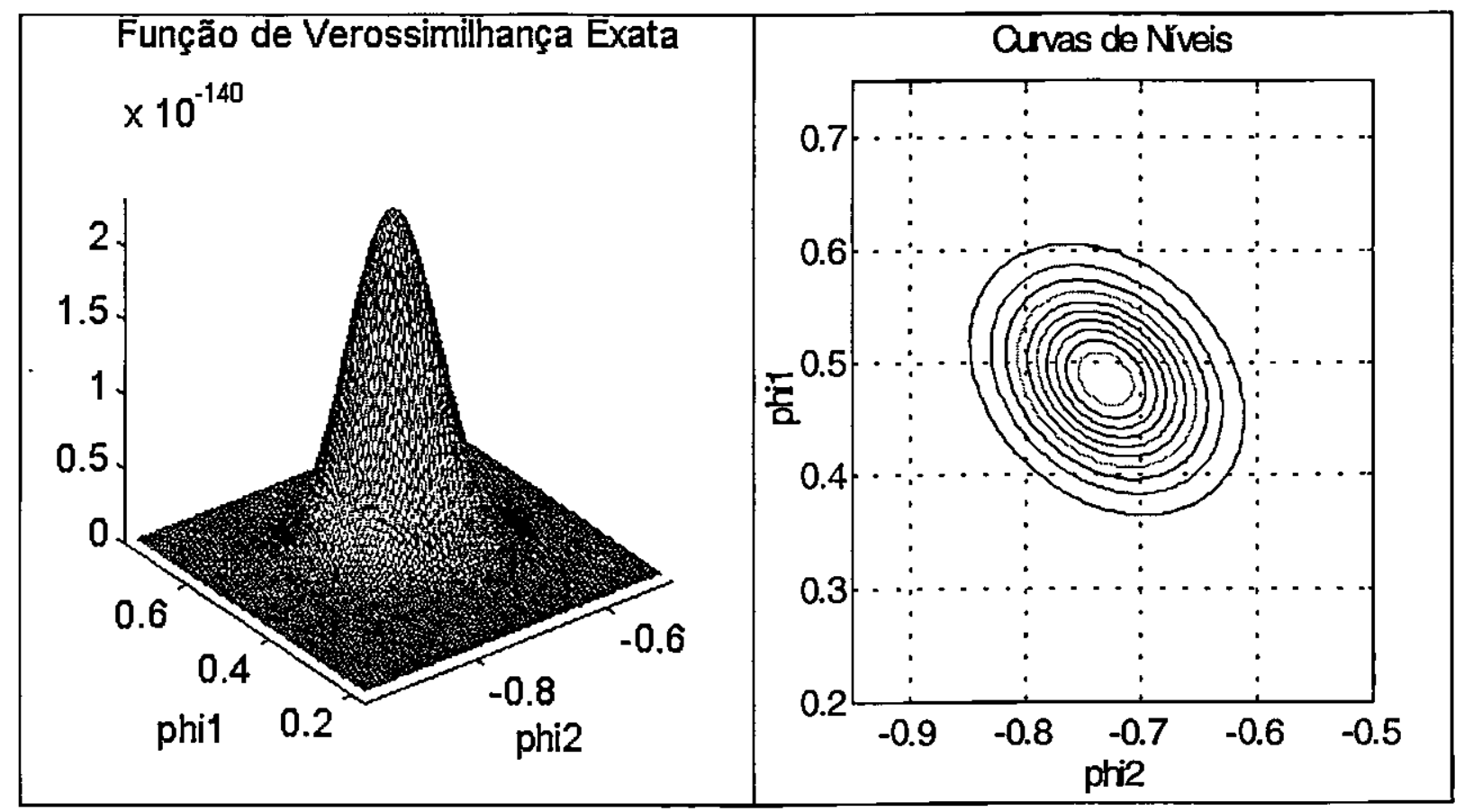

fig. 8 - Gráfico da função de verossimilhança e curvas de níveis para a série simulada $z_{t}=0.5 z_{t-1}-0.7 z_{t-2}+a_{t}$. 
Os resultados da aplicação do algoritmo Metropolis-Hastings são dados na tabela e gráficos a seguir.

Tabela 4 - Metropolis-Hastings para a série $\mathrm{z}_{t}=0.5 \mathrm{z}_{t-1}-0.7 \mathrm{z}_{t-2}+a_{t}$

\begin{tabular}{|l|c|c|c|}
\hline \multicolumn{1}{|c|}{} & & & \\
\hline Média & 0.23728 & 0.48466 & -0.73485 \\
\hline Moda (EMV) & 0.23668 & 0.48191 & -0.73807 \\
\hline Mediana & 0.23575 & 0.48364 & -0.73514 \\
\hline$\sqrt{\widehat{\mathrm{R}}}$ - (crit. de conv.) & 1.00434 & 0.99983 & 1.00028 \\
\hline
\end{tabular}

Neste caso também verificamos que o critéiro de Gelman e Rubin sugere a convergência com $\sqrt{\widehat{\mathrm{R}}}<1.01$.

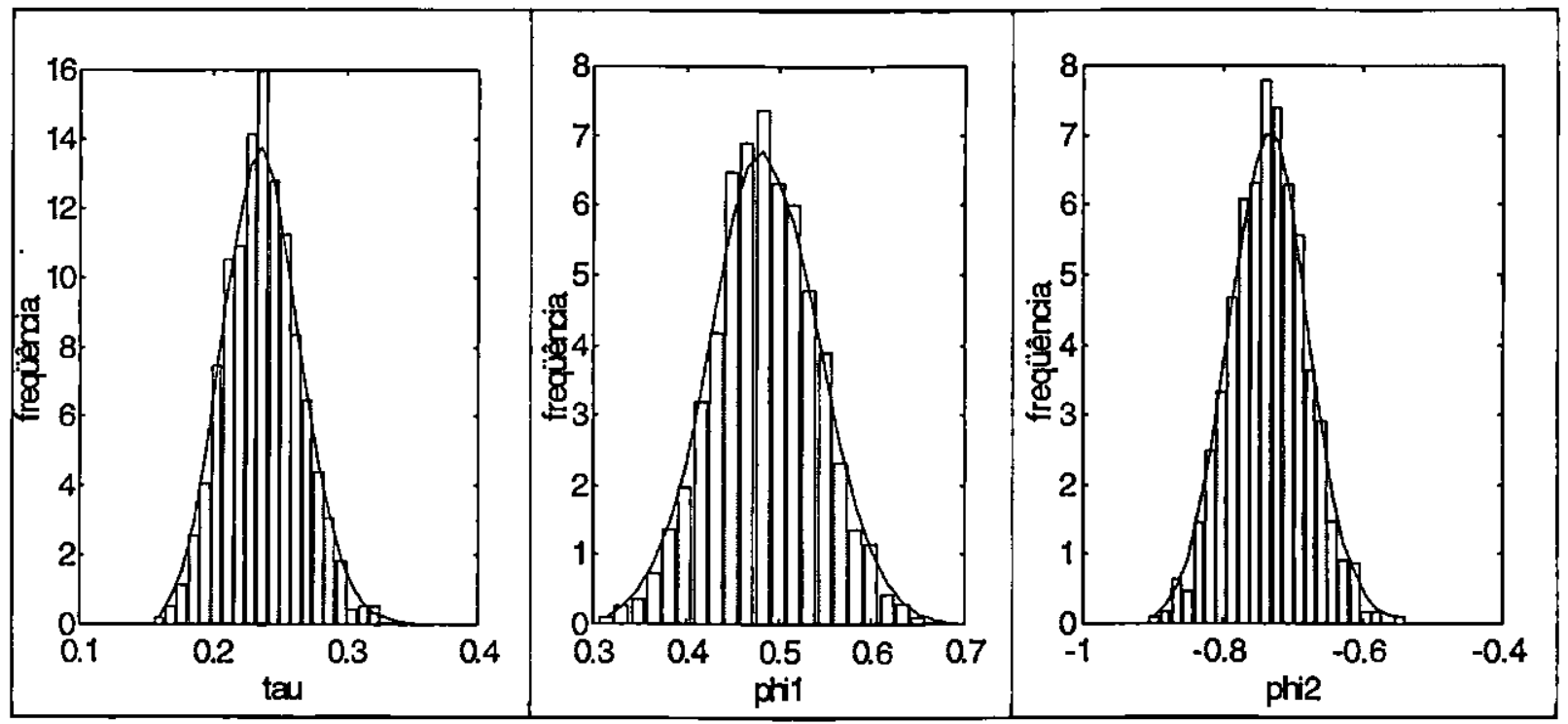

fig. 9 - Histogramas das distribuições aproximadas de $\tau, \phi_{1}$ e $\phi_{2}$. 

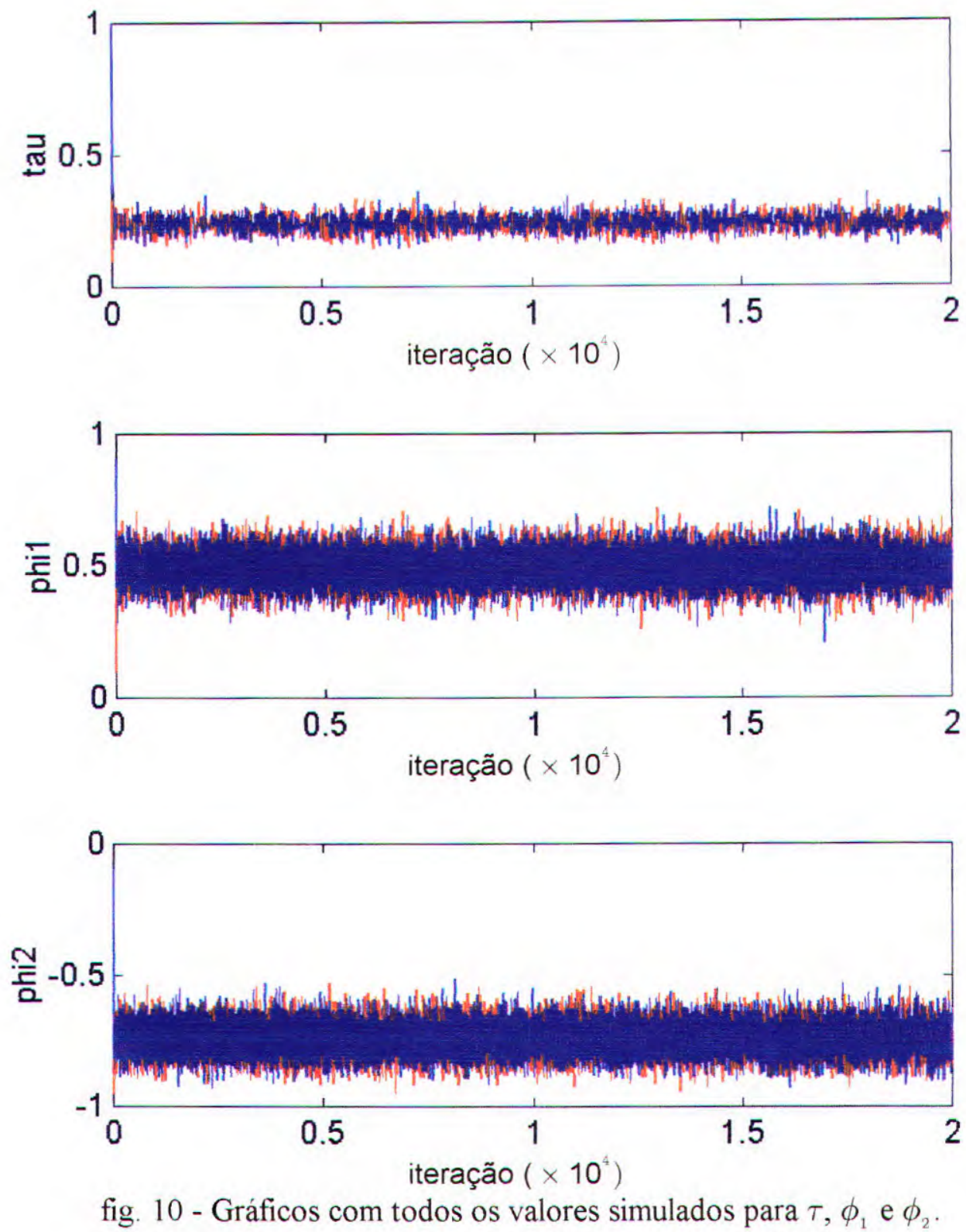


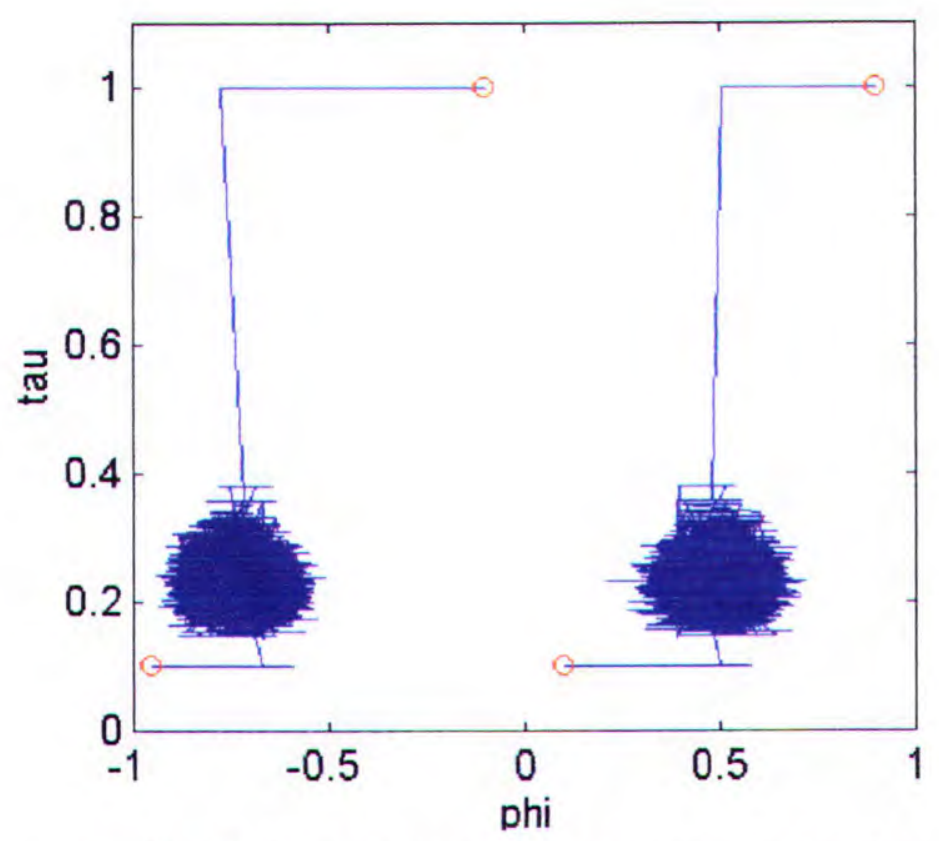

fig. 11 - Gráfico mostrando a convergência das duas cadeias geradas, com seus repectivos pontos de partida.

No gráfico da figura 11 temos no eixo horizontal $\phi_{1}$ e $\phi_{2}$ e no vertical $\tau$. Notamos neste gráfico a convergência do algoritmo para duas condições iniciais diferentes. 


\section{Série Furnas:}

Vamos mostrar aqui os resultados para a série Fumas com 576 observações. A seguir, apresentamos o gráfico da série e os resultados de cada método aplicado.

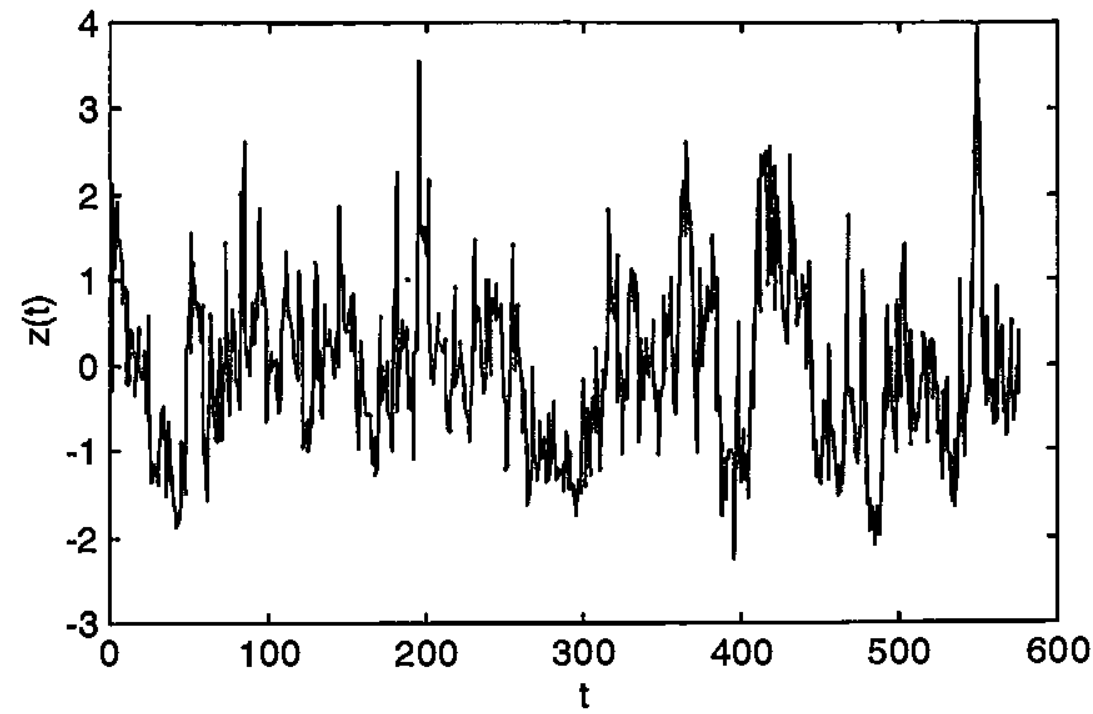

fig. 12 - Gráfico da série de dados reais Fumas com 576 observações.

Tabela 5 - Valores ajustados para a série Fumas

\begin{tabular}{|l|l|c|c|}
\hline \multicolumn{1}{|c|}{} & & & \\
\hline Estimat. Mínimos Quadrados & 2.20994 & 0.59602 & 0.17437 \\
\hline Aproximação das E.M.V. & 2.20993 & 0.59499 & 0.17376 \\
\hline ARMLE & 2.20993 & 0.59500 & 0.17376 \\
\hline Metropolis-Hastings & 2.20503 & 0.59736 & 0.17588 \\
\hline
\end{tabular}

Vemos que as estimativas dos métodos foram bastante próximas para todos os parâmetros.

Na figura seguinte, mostramos o gráfico da função de verossimilhança exata da série, construído com $\tau=2.21, \phi_{1}$ variando no intervalo $[0.46,0.71]$ e $\phi_{2}$ variando no intervalo $[0.03,0.29]$. 


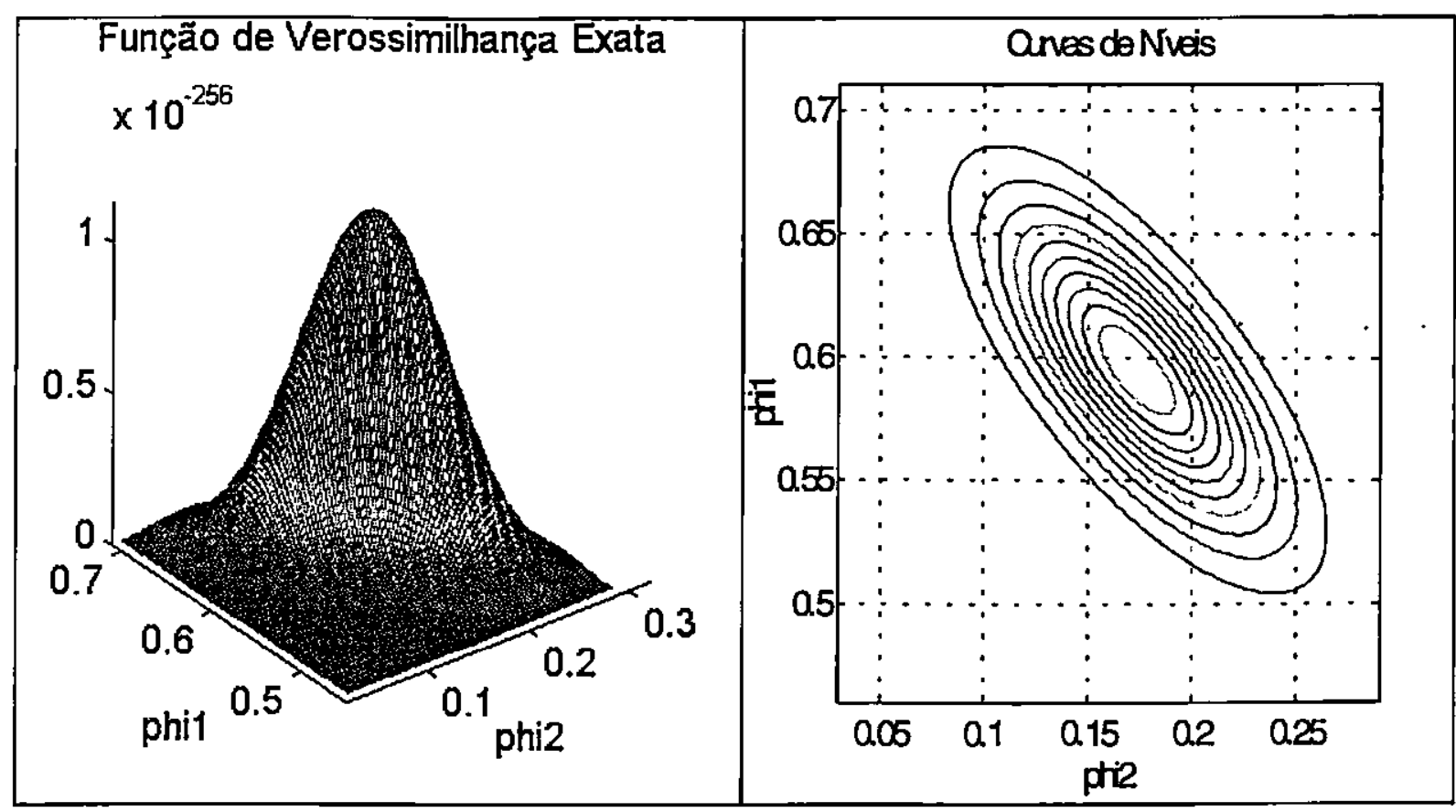

fig. 13 - Gráfico da função de verossimilhança e curvas de níveis para a série real Furnas.

Com as estimativas $\widehat{\phi}_{1}=0.59736 \mathrm{e} \widehat{\phi}_{2}=0.17588$ geradas por Metropolis-Hastings, calculamos previsões de 1 passo à frente para 36 meses referentes ao período de janeiro de 1979 a dezembro de 1981. Abaixo mostramos o gráfico com as previsões calculadas e os dados observados.

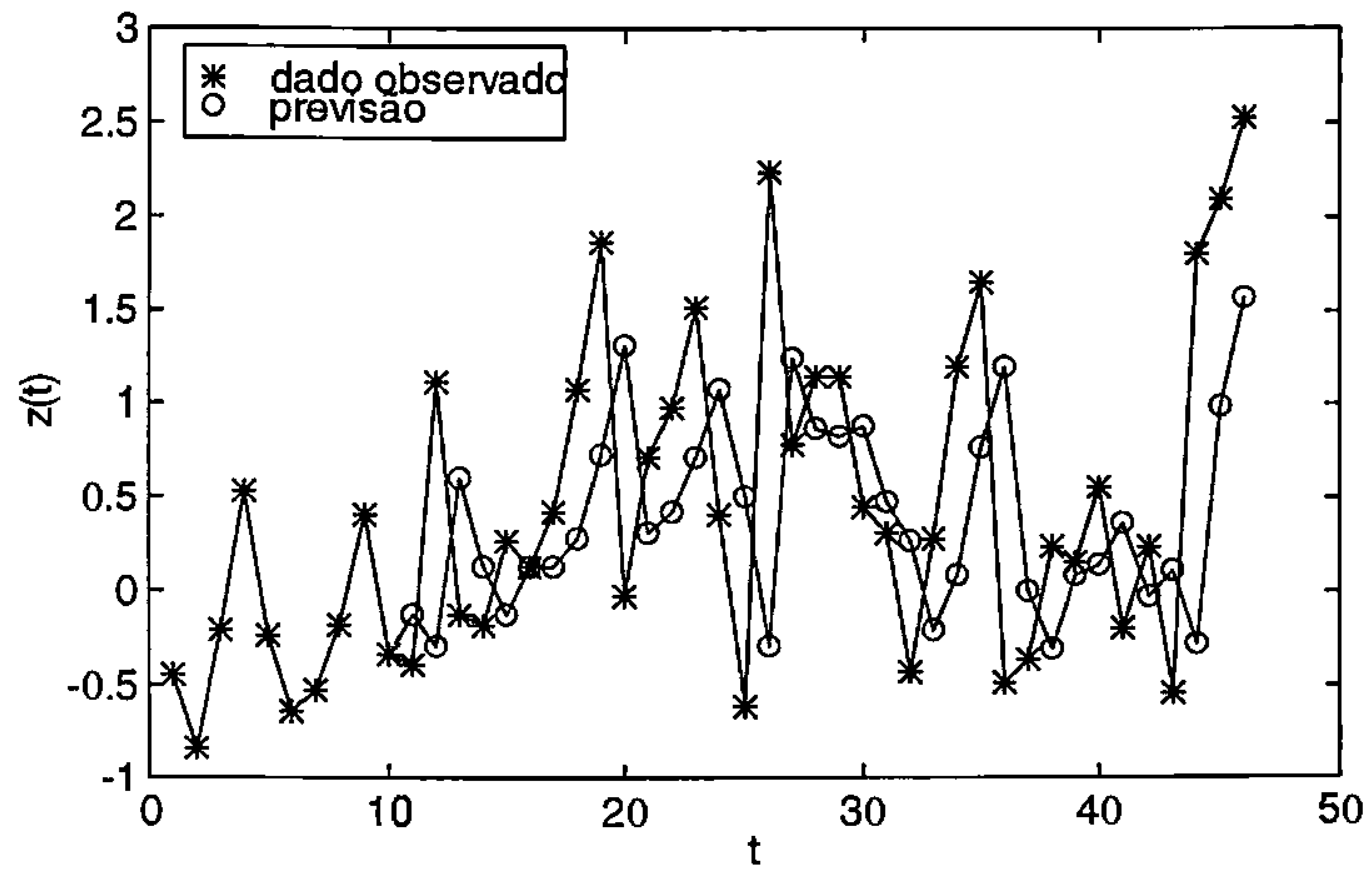

fig. 14 - Previsão 1 passo à frente para 36 dados da série Furnas. 
Um resumo dos resultados obtidos pelo algoritmo de Metropolis-Hastings são dados na tabela abaixo:

Tabela 6 - Metropolis-Hastings para a série Furnas

\begin{tabular}{|l|c|c|c|}
\hline \multicolumn{1}{|l|}{} & & & \\
\hline Média & 2.20562 & 0.59322 & 0.17575 \\
\hline Moda (EMV) & 2.20503 & 0.59736 & 0.17588 \\
\hline Mediana & 2.19634 & 0.59379 & 0.17525 \\
\hline$\sqrt{\widehat{R}}$ - (crit. de conv.) & 1.00462 & 1.00094 & 0.99986 \\
\hline
\end{tabular}

Os histogramas construídos com as amostras selecionadas para cada um dos parâmetros, assim como os gráficos de convergência são dados nas figuras seguintes.

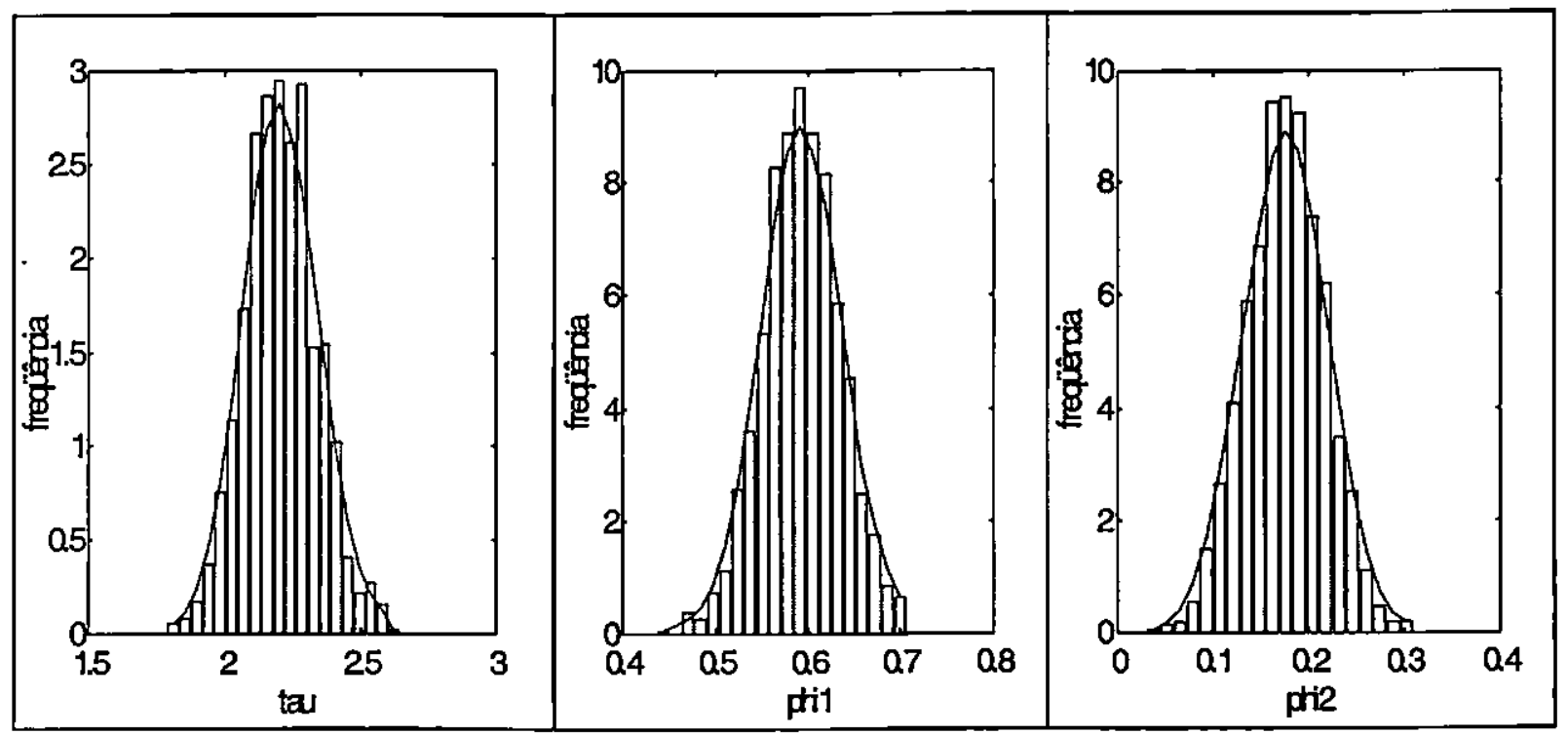

fig. 15 - Histogramas das distribuições aproximadas de $\tau, \phi_{1}$ e $\phi_{2}$. 

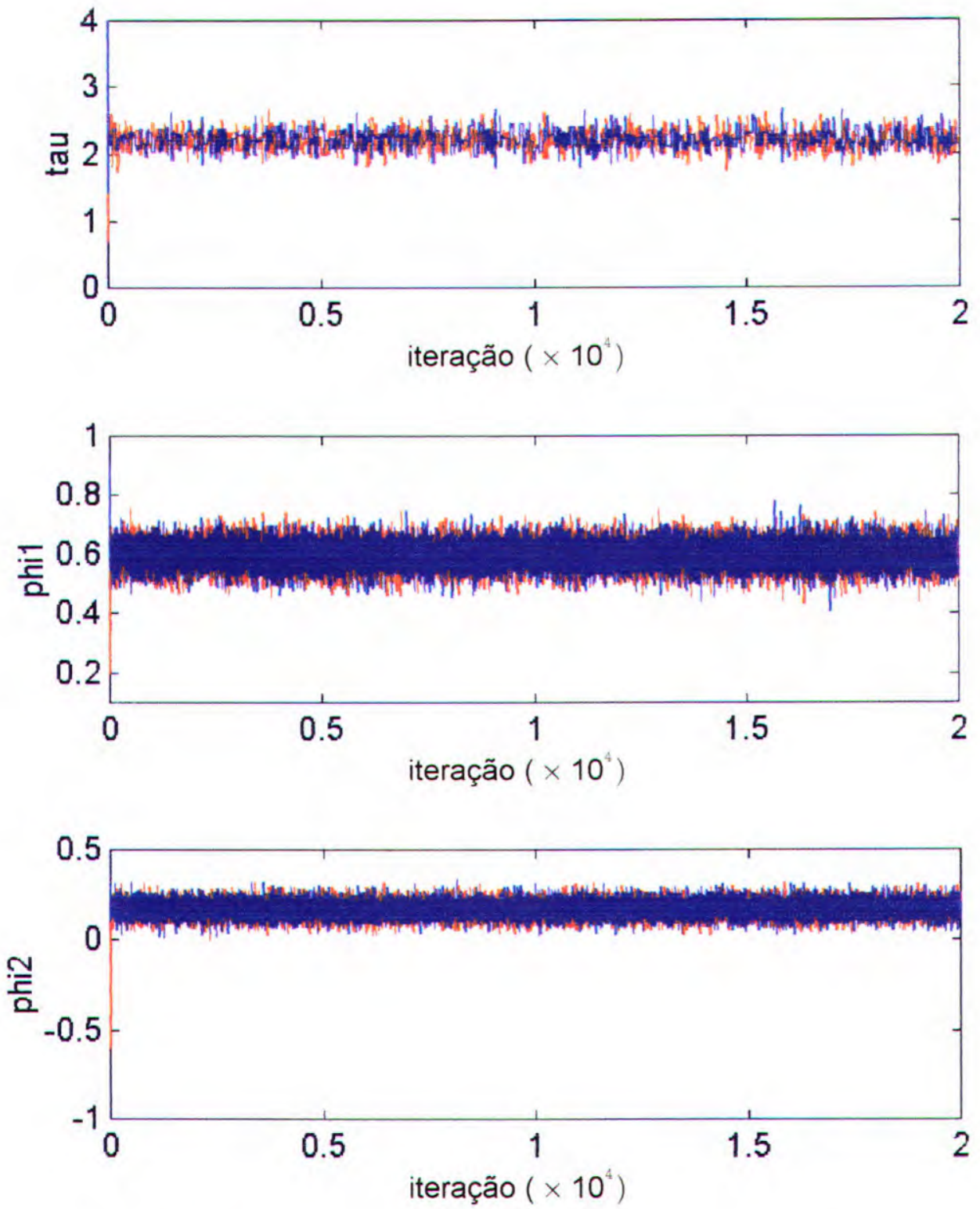

fig. 16 - Gráficos com os valores simulados para $\tau, \phi_{1}$ e $\phi_{2}$. 


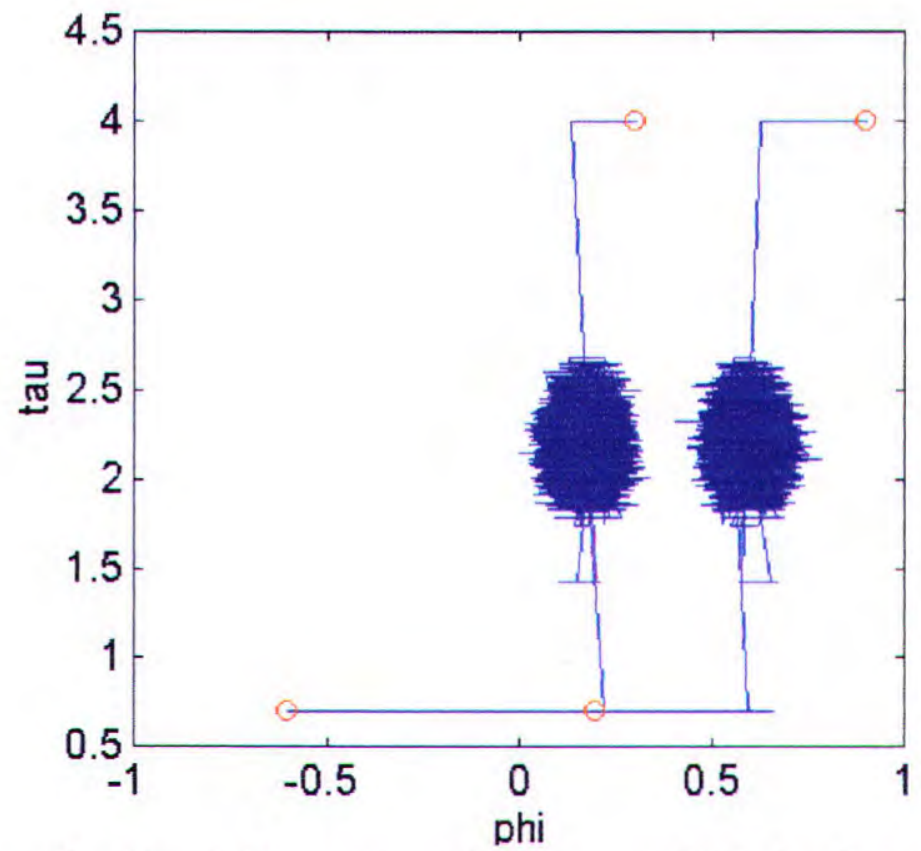

fig. 17 - Gráfico mostrando a convergência das 2 cadeias geradas, com seus repectivos pontos de partida. 


\subsection{Aplicação aos Modelos MA(q)}

Veremos aqui a aplicação para os modelos MA(1) e modelos $\mathrm{MA}(2)$ dos métodos numéricos de estimação de máxima verossimilhança vistos na seção 4.2 (estimação de máxima verossimilhança condicional e estimação de máxima verossimilhança incondicional) e também dos métodos de simulação de Monte Carlo vistos no capítulo 5. Para cada um dos modelos é dado também um exemplo utilizando-se séries simuladas.

\section{- Modelo MA(1):}

Suponhamos que o conjunto de observações $z_{n}^{\prime}=\left(z_{1}, z_{2}, \ldots, z_{n}\right)$ seja gerado pelo modelo de médias móveis invertível de ordem 1, ou MA(1),

$$
\mathrm{z}_{\mathrm{t}}=a_{\mathrm{t}}-\theta a_{\mathrm{t}-1}
$$

com $a_{t} \sim N\left(0, \tau^{-1}\right)$. A invertibilidade do modelo é garantida desde que o valor de $\theta$ satisfaça à condição $-1<\theta<1$.

Para estimar os parâmetros $\tau$ e $\theta$ através do primeiro método, devemos assumir como valor inicial $a_{*}=a_{0}=0$ e calcular a soma de quadrados $S_{*}(\theta)=\sum_{t=1}^{n} a_{t}^{2}\left(\theta \mid \mathbf{z}_{n}, a_{0}\right)$ para vários valores de $\theta$ no intervalo $(-1,1)$, sendo que para cada $\theta$ fixado os valores de $a_{\imath}$, para $t=1, \ldots, n$, são obtidos iterativamente fazendo

$$
\begin{gathered}
a_{1}=\mathrm{z}_{1}+\theta a_{0} \\
a_{2}=\mathrm{z}_{2}+\theta a_{1} \\
\vdots \\
a_{n}=\mathrm{z}_{n}+\theta a_{n-1}
\end{gathered}
$$

O valor de $\theta$ que minimizar $\mathrm{S}_{*}(\theta)$ será então o estimador de máxima verossimilhança $\widehat{\theta}$. Após obter $\widehat{\theta}$, a estimativa $\widehat{\tau}$ de $\tau$ pode ser encontrada de

$$
\widehat{\tau}^{-1}=\frac{S_{*}(\widehat{\theta})}{n-2}
$$


No segundo método, a estimativa $\widehat{\theta}$ é obtida minimizando a soma de quadrados incondicional $\mathrm{S}(\theta)=\sum_{t=0}^{n}\left[a_{t}\right]^{2}$, calculada para diversos valores de $\theta$ no intervalo $(-1,1)$. Para isso são necessários dois processos iterativos. No primeiro, assumimos $\left[e_{n+1}\right]=0$ e sabendo que $\left[\mathrm{z}_{t}\right]=\mathrm{z}_{t}$, para $t=1, \ldots, n$, fazemos

$$
\begin{gathered}
{\left[e_{n}\right]=\left[\mathbf{z}_{n}\right]+\theta\left[e_{n+1}\right]} \\
{\left[e_{n-1}\right]=\left[\mathbf{z}_{n-1}\right]+\theta\left[e_{n}\right]} \\
\vdots \\
{\left[e_{0}\right]=\left[\mathbf{z}_{0}\right]+\theta\left[e_{1}\right]}
\end{gathered}
$$

Da última expressão, tiramos o valor de $\left[\mathbf{z}_{0}\right]$, pois $\left[e_{0}\right]=0$, e iniciamos então o segundo processo iterativo fazendo

$$
\begin{aligned}
{\left[a_{0}\right] } & =\left[\mathbf{z}_{0}\right]+\theta\left[a_{-1}\right] \\
{\left[a_{1}\right] } & =\left[\mathbf{z}_{1}\right]+\theta\left[a_{0}\right] \\
& \vdots \\
{\left[a_{n}\right] } & =\left[\mathbf{z}_{n}\right]+\theta\left[a_{n-1}\right]
\end{aligned}
$$

levando em consideração que $\left[a_{-1}\right]=0$. Repetimos este procedimento para todos os valores de $\theta$ e calculamos então $\widehat{\theta}$. A estimativa de $\tau$ é calculada a partir de $\widehat{\theta}$ através de

$$
\widehat{\tau}^{-1}=\frac{S(\widehat{\theta})}{n}
$$

Para a aplicação dos algoritmos MCMC, devemos utilizar a função de verossimilhança exata para modelos MA(q), definida na seção 5.6:

$$
\begin{aligned}
L\left(\boldsymbol{\theta}, \tau \mid \mathbf{z}_{n}, \boldsymbol{a}_{*}\right)= & \left(\frac{\tau}{2 \pi}\right)^{n / 2} \exp \left\{-\frac{\tau}{2}\left[\left(\mathbf{z}_{n}-\boldsymbol{F} \boldsymbol{a}_{*}\right)^{\prime} \boldsymbol{G}^{\prime^{-1}} \boldsymbol{G}^{-1}\left(\mathbf{z}_{n}-\boldsymbol{F} \boldsymbol{a}_{*}\right)\right]\right\} \\
& \cdot\left(\frac{\tau}{2 \pi}\right)^{q / 2} \exp \left\{-\frac{\tau}{2} \boldsymbol{a}_{*}^{\prime} \boldsymbol{a}_{*}\right\}
\end{aligned}
$$


onde, para o caso MA(1), teremos:

$$
\begin{aligned}
& L\left(\theta, \tau \mid \mathbf{z}_{n}, a_{*}\right)=\left(\frac{\tau}{2 \pi}\right)^{n / 2} \exp \left\{-\frac{\tau}{2}\left[\left(\mathbf{z}_{n}-\boldsymbol{F} a_{*}\right)^{\prime} \boldsymbol{G}^{\prime-1} \boldsymbol{G}^{-1}\left(\mathbf{z}_{n}-\boldsymbol{F} \boldsymbol{a}_{*}\right)\right]\right\} \\
& \cdot\left(\frac{\tau}{2 \pi}\right)^{1 / 2} \exp \left\{-\frac{\tau}{2} a_{*}^{\prime} a_{*}\right\}
\end{aligned}
$$

com

$$
\boldsymbol{a}_{*}=a_{0}, \quad \boldsymbol{G}=\left[\begin{array}{cccccc}
1 & 0 & 0 & \cdots & 0 & 0 \\
-\theta & 1 & 0 & \cdots & 0 & 0 \\
0 & -\theta & 1 & \cdots & 0 & 0 \\
\vdots & \vdots & \vdots & \cdots & \vdots & \vdots \\
0 & 0 & 0 & \cdots & -\theta & 1
\end{array}\right]_{n \times n} \quad \text { e } \quad \boldsymbol{F}=\left[\begin{array}{c}
-\theta \\
0 \\
0 \\
\vdots \\
0
\end{array}\right]_{n \times 1}
$$

Então, as amostras para $a_{0}, \tau$ e $\theta$ são simuladas iterativamente a partir das densidades

$$
\begin{aligned}
a_{0} & \sim N\left(0, \tau^{-1}\right) \\
\tau & \sim \Gamma\left(\frac{n+1}{2}+1, \frac{\mathrm{S}\left(\theta, a_{0}\right)}{2}\right) \\
\theta & \sim N\left(\mu_{\theta}, \sigma_{\theta}^{2}\right)
\end{aligned}
$$

onde

$$
\mathrm{S}\left(\theta, a_{0}\right)=\left(\mathbf{z}_{n}-\boldsymbol{F} a_{0}\right)^{\prime} \boldsymbol{G}^{\prime^{-1}} \boldsymbol{G}^{-1}\left(\mathbf{z}_{\mathrm{n}}-\boldsymbol{F} a_{0}\right)+a_{0}^{\prime} a_{0}
$$

e os parâmetros da distribuição normal $\mu_{\theta}$ e $\sigma_{\theta}^{2}$ devem ser ajustados de modo que a taxa de aceitação dos valores simulados não seja muito baixa. Note que para simular $a_{0}$ e $\tau$ utilizamos o amostrador de Gibbs e para $\theta$ utilizamos o algoritmo de Metropolis-Hastings.

Vamos agora exemplificar a aplicação dos métodos em uma série gerada pelo modelo $\operatorname{MA}(1): \mathrm{z}_{t}=a_{t}-0.8 a_{t-1}, \operatorname{com} a_{t} \sim N(0,1)$. Foram simulados 100 pontos e o gráfico da série é apresentado na figura seguinte: 


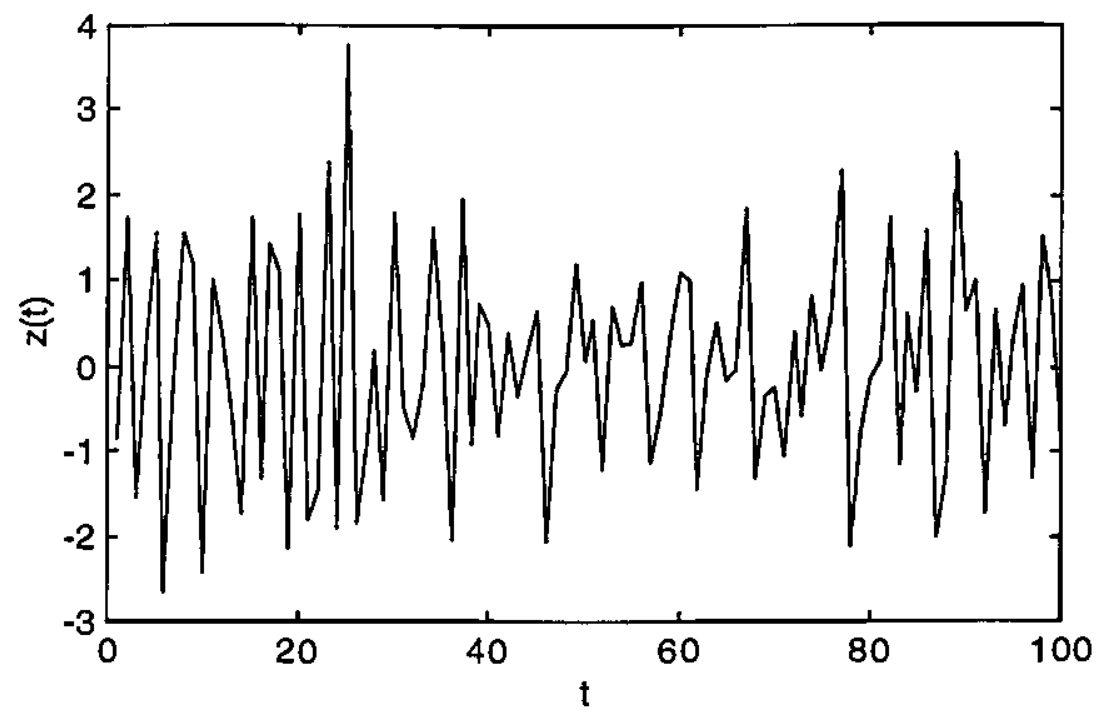

fig. 18 - Gráfico da série simulada $\mathrm{z}_{t}=a_{t}-0.8 a_{t-1}, a_{t} \sim N(0,1)$.

Após aplicar os três métodos para a série, obtivemos as seguintes estimativas:

Tabela 7 - Resultados das estimativas para os parâmetros da série.

\begin{tabular}{|l|c|c|}
\hline Est. de Máxima Verossimilhança Condicional & 0.93183 & 0.78670 \\
\hline Est. de Máxima Verossimilhança Incondicional & 0.95221 & 0.78970 \\
\hline Gibbs / Metropolis-Hastings & 0.96231 & 0.75641 \\
\hline
\end{tabular}

Comparando-se os resultados, vemos que no caso do parâmetro $\tau$ a estimativa obtida por MCMC se apresentou um pouco mais próxima do valor real $\tau=1$, mas para o parâmetro $\theta$ os métodos numéricos apresentaram um resultado melhor.

A seguir, mostramos o gráfico gerado através da expressão da função de verossimilhança exata para um modelo MA(1), construída a partir dos dados da série e variando $\tau$ no intervalo $[0.5,1.5]$ e $\theta$ no intervalo $[0.49,0.99]$. 


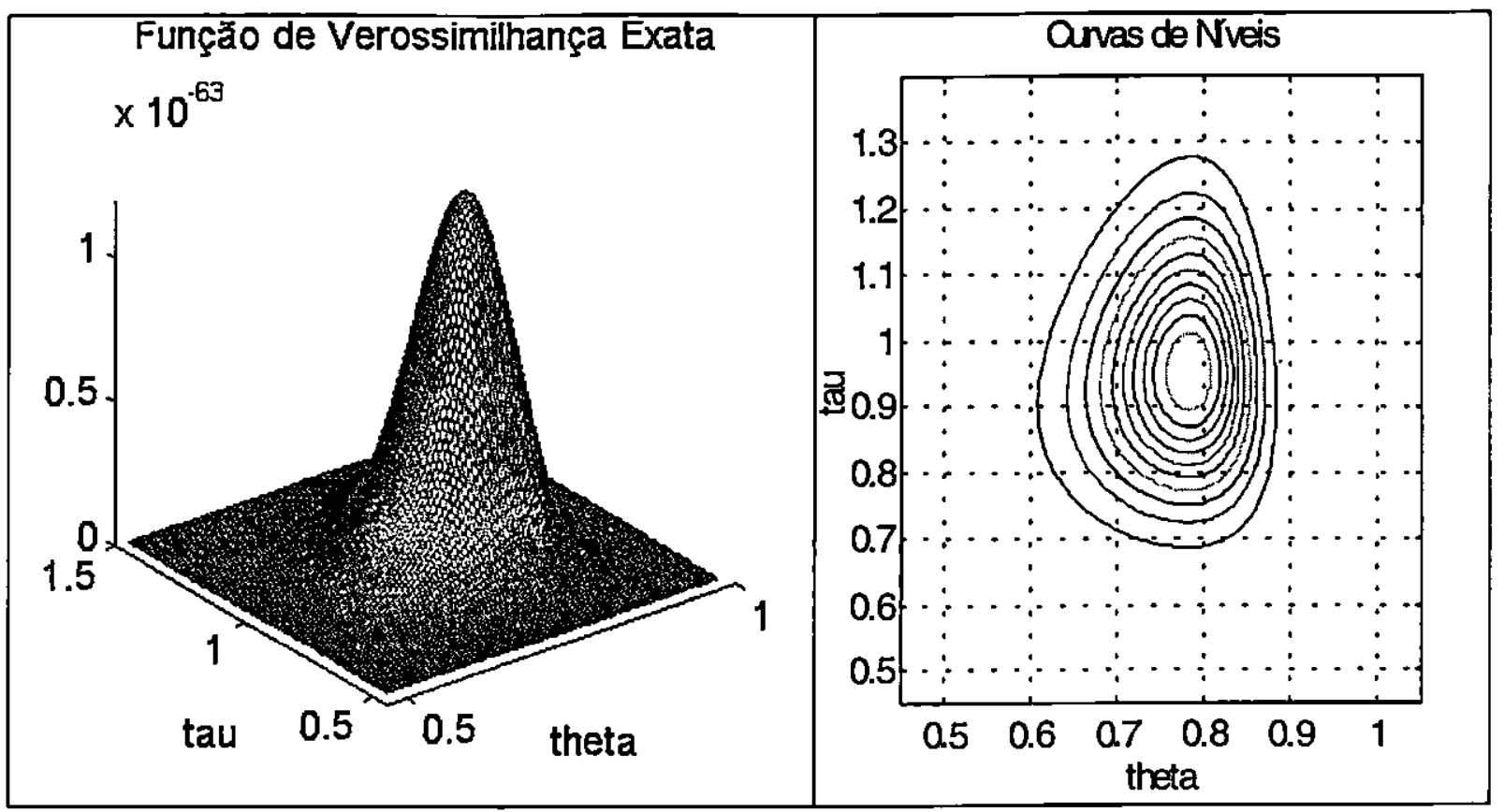

fig. 19 - Gráfico da função de verossimilhança e curvas de níveis para a série simulada $\mathrm{z}_{t}=a_{i}-0.8 a_{i-1}, a_{i} \sim N(0,1)$.

$\mathrm{Na}$ implementação dos dois primeiros métodos, foram dados valores para $\theta$ espaçados de $10^{-4}$ no intervalo $[-0.99,0.99]$. Para o método MCMC, foram simuladas 2 cadeias com 15000 iteraçōes cada, para cada um dos parâmetros. A seleção da amostra final foi realizada descartando-se parte inicial de cada cadeia (correspondente a $30 \%$ de cada uma) e tomando-se valores espaçados de 10 iterações (devido à correlação entre os valores simulados), totalizando assim 2100 amostras para cada parâmetro. Para a geração dos candidatos de $\theta$ no caso do uso do algoritmo de Metropolis-Hastings, ajustamos como núcleo uma distribuição normal $N(0.7,0.05)$. Os resultados para $\tau$ e $\theta$ após simular Gibbs/Metropolis-Hastings, foram:

Tabela 8 - Gibbs / Metropolis-Hastings para a série simulada.

\begin{tabular}{|l|l|l|}
\hline \multicolumn{1}{|c|}{} & 0.94033 & 0.74212 \\
\hline Média & 0.96231 & 0.75641 \\
\hline Moda (EMV) & 0.93664 & 0.75089 \\
\hline Mediana & 1.00064 & 1.00110 \\
\hline$\sqrt{\widehat{\mathrm{R}}}$ - (crit. de converg.) \\
\hline
\end{tabular}


Nas figuras seguintes, apresentamos os histogramas construídos com a amostra final selecionada para cada parâmetro e os gráficos de convergência das duas cadeias com todos os pontos simulados pelo algoritmo Gibbs/Metropolis-Hastings.

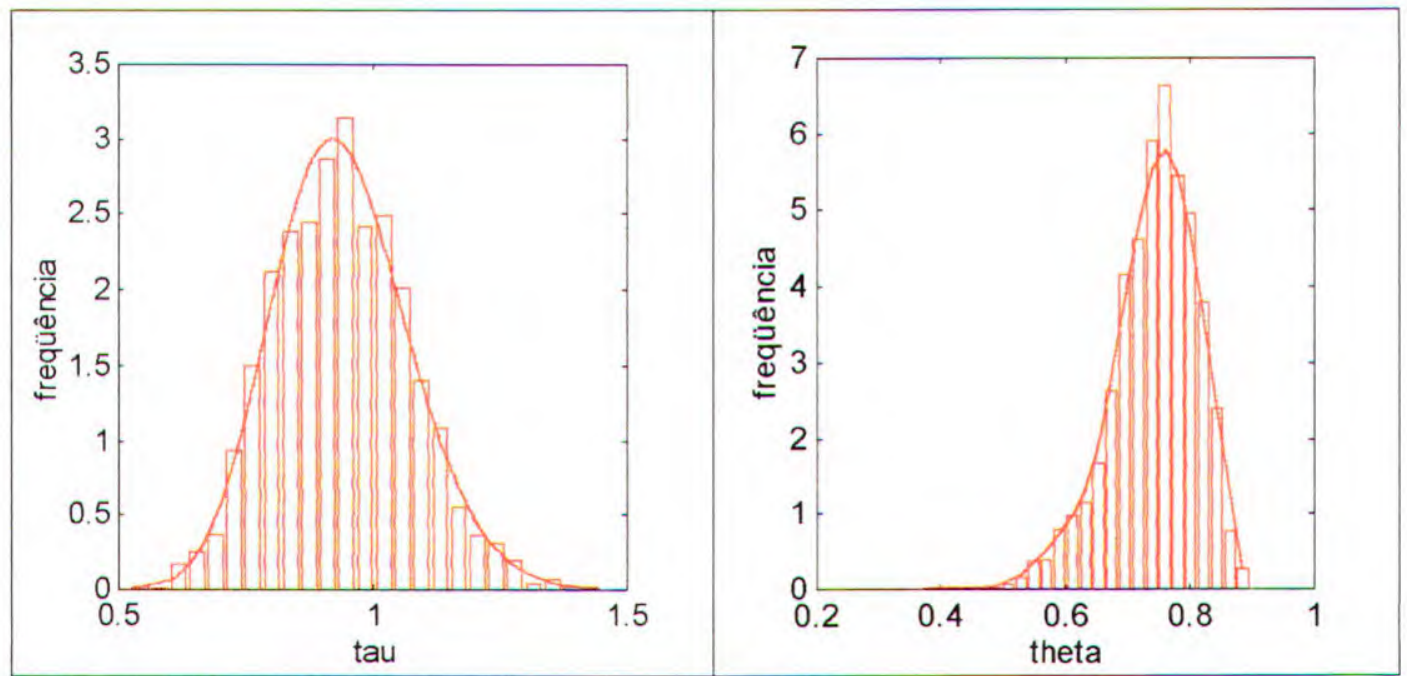

fig. 20 - Histogramas das amostras selecionadas geradas por MCMC para $\tau$ e $\theta$.
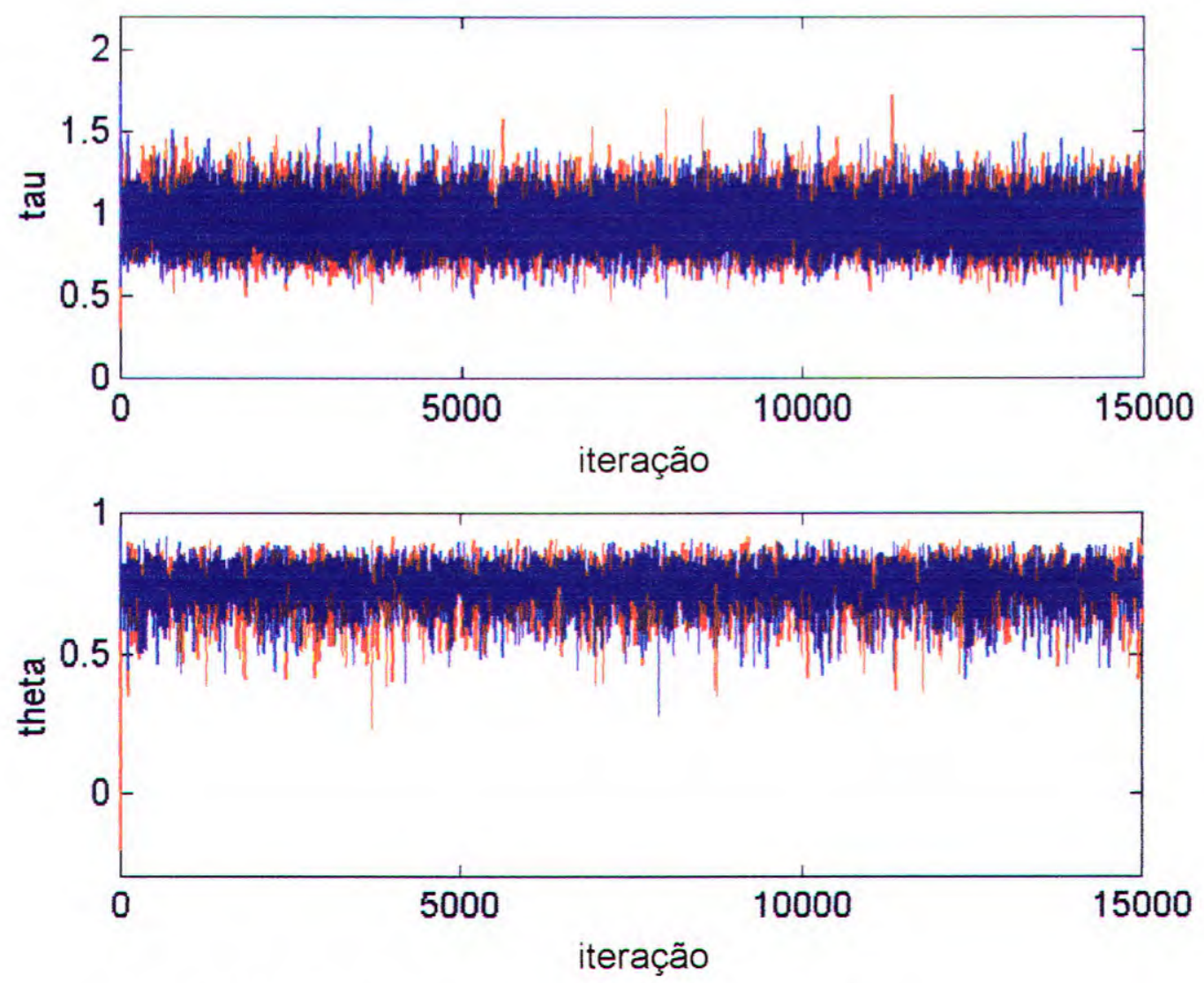

fig. 21 - Gráficos com os valores simulados para $\tau$ e $\theta$. 


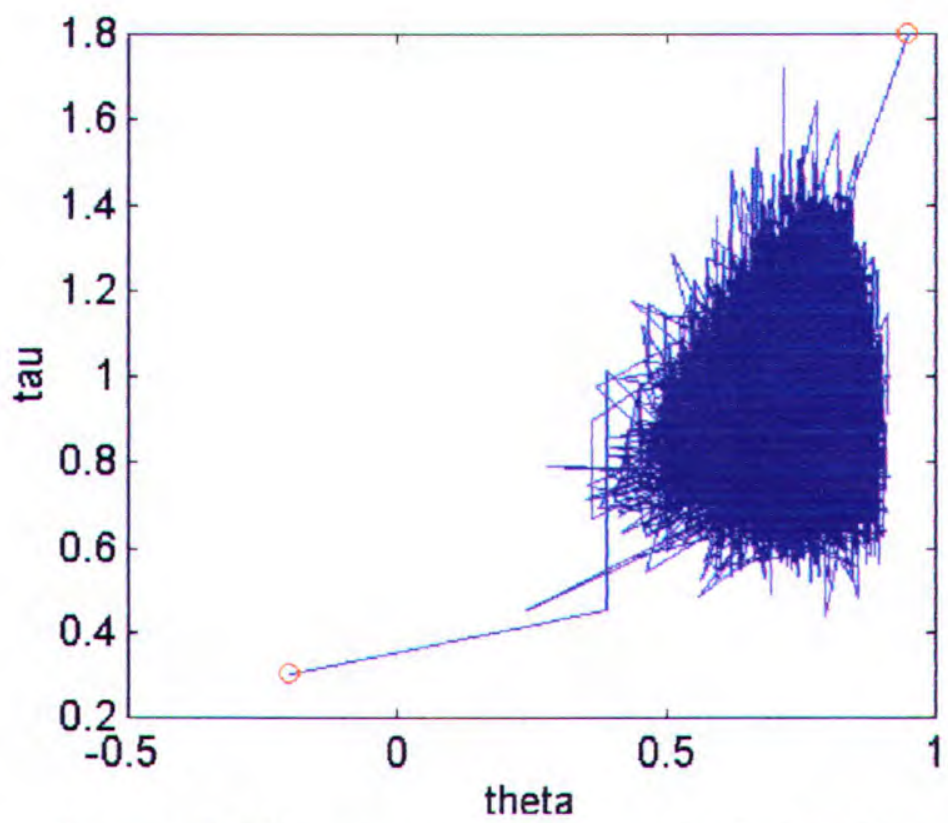

fig. 22 - Gráfico mostrando a convergência das duas cadeias geradas, com seus repectivos pontos de partida.

\section{- Modelo MA(2):}

Considere o modelo de médias móveis de ordem 2 invertível, dado por

$$
\mathrm{z}_{t}=a_{t}-\theta_{1} a_{t-1}-\theta_{2} a_{t-2}
$$

onde $a_{t} \sim N\left(0, \tau^{-1}\right)$. Para se garantir a invertibilidade, é necessário que $\theta_{1}$ e $\theta_{2}$ satisfaçam as seguintes condições: $\theta_{2}+\theta_{1}<1, \theta_{2}-\theta_{1}<1$ e $-1<\theta_{2}<1$. Suponha que as observações dadas $\mathbf{z}_{n}^{\prime}=\left(\mathrm{z}_{1}, \mathrm{z}_{2}, \ldots, \mathbf{z}_{n}\right)$ sejam geradas pelo modelo acima.

A estimação dos parâmetros $\tau$ e $\theta$ através do primeiro método é iniciada fixando valores para $\theta_{1}$ e $\theta_{2}$, e calculando $a_{t}$, para $t=1, \ldots, n$, iterativamente das expressões 


$$
\begin{aligned}
& a_{1}=\mathrm{z}_{1}+\theta_{1} a_{0}+\theta_{2} a_{-1} \\
& a_{2}=\mathrm{z}_{2}+\theta_{1} a_{1}+\theta_{2} a_{0} \\
& \quad \vdots \\
& a_{n}=z_{n}+\theta_{1} a_{n-1}+\theta_{2} a_{n-2}
\end{aligned}
$$

assumindo que $a_{n}=a_{-1}=0$. Feito isso, calculamos o valor de $S_{*}(\theta)=\sum_{t=1}^{n} a_{t}^{2}\left(\theta \mid \mathbf{z}_{n}, a_{*}\right)$, onde $\theta=\left(\theta_{1}, \theta_{2}\right)$, e repetimos o processo para diversos valores de $\theta_{1}$ e $\theta_{2}$. O estimador de máxima verossimilhança $\widehat{\theta}$ será então o valor de $\theta$ que minimizar $S_{*}(\theta)$ e a estimativa $\widehat{\tau}$ de $\tau$ será calculada de

$$
\widehat{\tau}^{-1}=\frac{S_{*}(\widehat{\theta})}{n-3}
$$

Para utilizar o segundo método devemos assumir $\left[e_{n+1}\right]=\left[e_{n+2}\right]=0$ e, usando o fato que $\left[z_{t}\right]=z_{t}$, para $t=1, \ldots, n$, calculamos para valores fixados de $\theta_{1}$ e $\theta_{2}$ as seguintes expressões:

$$
\begin{aligned}
{\left[e_{n}\right] } & =\left[\mathrm{z}_{n}\right]+\theta_{1}\left[e_{n+1}\right]+\theta_{2}\left[e_{n+2}\right] \\
{\left[e_{n-1}\right] } & =\left[\mathrm{z}_{n-1}\right]+\theta_{1}\left[e_{n}\right]+\theta_{2}\left[e_{n+1}\right] \\
& \vdots \\
{\left[e_{0}\right] } & =\left[\mathrm{z}_{0}\right]+\theta_{1}\left[e_{1}\right]+\theta_{2}\left[e_{2}\right] \\
{\left[e_{-1}\right] } & =\left[\mathrm{z}_{-1}\right]+\theta_{1}\left[e_{0}\right]+\theta_{2}\left[e_{1}\right]
\end{aligned}
$$

Nas duas últimas expressões, como $\left[e_{0}\right]=\left[e_{-1}\right]=0$, podemos tirar os valores para $\left[\mathrm{z}_{0}\right]$ e $\left[z_{-1}\right]$ e iniciar o segundo processo iterativo formado pelas equações

$$
\begin{aligned}
{\left[a_{-1}\right] } & =\left[\mathrm{z}_{-1}\right]+\theta_{1}\left[a_{-2}\right]+\theta_{2}\left[a_{-3}\right] \\
{\left[a_{0}\right] } & =\left[\mathrm{z}_{0}\right]+\theta_{1}\left[a_{-1}\right]+\theta_{2}\left[a_{-2}\right] \\
& \vdots \\
{\left[a_{n}\right] } & =\left[\mathrm{z}_{n}\right]+\theta_{1}\left[a_{n-1}\right]+\theta_{2}\left[a_{n-2}\right]
\end{aligned}
$$


usando $\circ$ fato que $\left[a_{-2}\right]=\left[a_{-3}\right]=0$. Em seguida, calculamos a soma de quadrados incondicional $S(\theta)=\sum_{t=-1}^{n}\left[a_{t}\right]^{2}$ e repetimos o processo para vários valores de $\theta_{1}$ e $\theta_{2}$. Feito isto, obtemos a estimativa de máxima verossimilhança $\widehat{\theta}$ minimizando $S(\theta)$ e a estimativa $\widehat{\tau}$ da equação

$$
\widehat{\tau}^{-1}=\frac{S(\widehat{\theta})}{n}
$$

Para a aplicação dos algoritmos MCMC, a função de verossimilhança exata para o modelo MA(2), é:

$$
\begin{aligned}
L\left(\boldsymbol{\theta}, \tau \mid \mathbf{z}_{n}, a_{*}\right)= & \left(\frac{\tau}{2 \pi}\right)^{n / 2} \exp \left\{-\frac{\tau}{2}\left[\left(\mathbf{z}_{n}-\boldsymbol{F} a_{*}\right)^{\prime} \boldsymbol{G}^{\prime^{-1}} \boldsymbol{G}^{-1}\left(\mathbf{z}_{n}-\boldsymbol{F} \boldsymbol{a}_{*}\right)\right]\right\} \\
& \cdot\left(\frac{\tau}{2 \pi}\right) \exp \left\{-\frac{\tau}{2} \boldsymbol{a}_{*}^{\prime} a_{*}\right\}
\end{aligned}
$$

onde

$$
\begin{aligned}
a_{*} & =\left(a_{-1}, a_{0}\right)^{\prime}, \\
G & =\left[\begin{array}{cccccccc}
1 & 0 & 0 & 0 & \cdots & 0 & 0 & 0 \\
-\theta_{1} & 1 & 0 & 0 & \cdots & 0 & 0 & 0 \\
-\theta_{2} & -\theta_{1} & 1 & 0 & \cdots & 0 & 0 & 0 \\
0 & -\theta_{2} & -\theta_{1} & 1 & \cdots & 0 & 0 & 0 \\
\vdots & \vdots & \vdots & \vdots & \cdots & \vdots & \vdots & \vdots \\
0 & 0 & 0 & 0 & \cdots & -\theta_{2} & -\theta_{1} & 1
\end{array}\right]_{n \times n}
\end{aligned}
$$

e

$$
\boldsymbol{F}=\left[\begin{array}{cc}
-\theta_{2} & -\theta_{1} \\
0 & -\theta_{2} \\
0 & 0 \\
0 & 0 \\
\vdots & \vdots \\
0 & 0
\end{array}\right]_{n \times 2}
$$


Vimos na seção 5.6 que as amostras para $a_{*}, \tau$ e $\theta$ são simuladas iterativamente a partir das densidades

$$
\begin{aligned}
& a_{*} \sim N\left(0, \tau^{-1}\right) \\
& \tau \sim \Gamma\left(\frac{n+2}{2}+1, \frac{S\left(\theta, a_{*}\right)}{2}\right) \\
& \theta \sim N\left(\mu_{\theta}, \sigma_{\theta}^{2}\right)
\end{aligned}
$$

onde

$$
S\left(\theta, a_{*}\right)=\left(\mathbf{z}_{n}-F a_{*}\right)^{\prime} G^{\prime^{-1}} G^{-1}\left(\mathbf{z}_{n}-F a_{*}\right)+a_{*}^{\prime} a_{*}
$$

e os parâmetros $\mu_{\theta}$ e $\sigma_{\theta}^{2}$ da última distribuição normal, devem ser ajustados de acordo com a taxa de aceitação dos valores simulados. A simulação de $a_{*}$ e $\tau$ é feita utilizando o amostrador de Gibbs e para $\theta$ utilizamos o algoritmo de Metropolis-Hastings.

Ilustraremos agora a aplicação de cada um dos métodos na série de 100 observações simuladas pelo modelo $z_{t}=a_{t}-0.8 a_{t-1}+0.6 a_{t-2}$, onde $a_{t} \sim N(0,1)$.

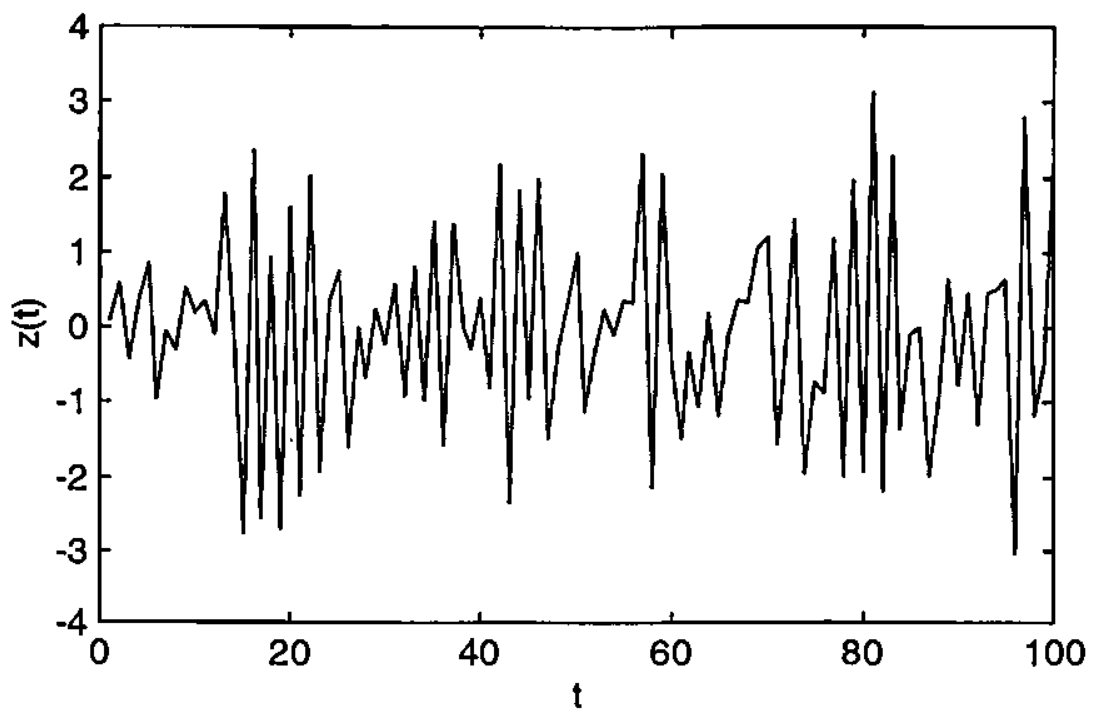

fig. 23 - Gráfico da série simulada $z_{t}=a_{t}-0.8 a_{t-1}+0.6 a_{t-2}$. 
Para cada um dos métodos estudados, as estimativas calculadas para $\tau, \theta_{1}$ e $\theta_{2}$ são dadas na tabela abaixo.

Tabela 9 - Resultados das estimativas para os parâmetros da série.

\begin{tabular}{|l|c|c|c|}
\hline \multicolumn{1}{|c|}{ Métodos } & $\tau$ & $\theta_{1}$ & $\theta_{2}$ \\
\hline Est. de Máxima Verossimilhança Condicional & 0.97237 & 0.78550 & -0.64290 \\
\hline Est. de Máxima Verossimilhança Incondicional & 1.00256 & 0.78630 & -0.64340 \\
\hline Gibbs / Metropolis-Hastings & 0.99749 & 0.71545 & -0.56344 \\
\hline
\end{tabular}

Analisando os resultados, vemos que para $\tau$ as estimativas dos dois últimos métodos foram bastante próximas do valor real $\tau=1$ e que para $\theta_{1}$ e $\theta_{2}$ as estimativas do método MCMC foram mais baixas do que os métodos numéricos.

Na figura seguinte, mostramos o gráfico da função de verossimilhança exata da série, obtido variando-se $\theta_{1}$ no intervalo $[0.3,1.1], \theta_{2}$ no intervalo $[-0.95,-0.15]$ e fixando $\tau=1$.

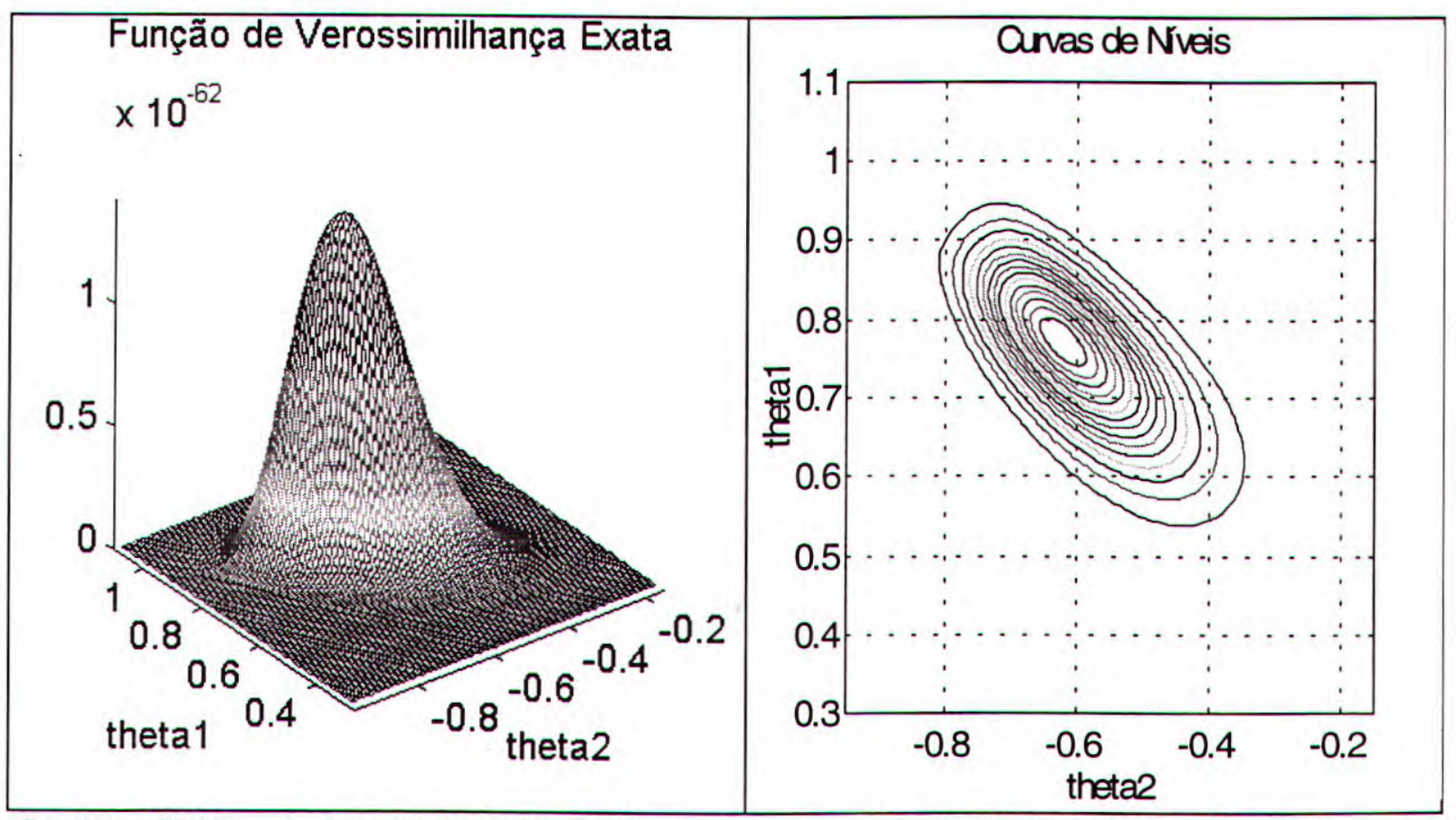

fig. 24 - Gráfico da função de verossimilhança e curvas de níveis para a série simulada $\mathrm{z}_{t}=a_{t}-0.8 a_{t-1}+0.6 a_{t-2} \operatorname{com} a_{t} \sim N(0,1)$. 
Para os métodos numéricos, o incremento usado para $\theta_{1}$ e $\theta_{2}$ foi de $10^{-4} . \mathrm{Na}$ implementação de Gibbs/Metropolis-Hastings, o número de simulações e a metodologia usada para a seleção da amostra final foi a mesma que a utilizada para o caso MA(1). No uso do algoritmo de Metropolis-Hastings para a geração dos candidatos de $\theta$, ajustamos como núcleo uma normal com média $\mu_{\theta}=(0.7,-0.5)$ e variância $\sigma_{\theta}^{2}=0.01$. Um resumo dos resultados obtidos pelo algoritmo são dados na tabela abaixo.

Tabela 10 - Gibbs/Metropolis-Hastings para a série $z_{t}=a_{t}-0.8 a_{t-1}+0.6 a_{t-2}$.

\begin{tabular}{|l|c|c|c|}
\hline \multicolumn{1}{|c|}{} & 0.98540 & 0.71043 & -0.53684 \\
\hline Média & 0.99749 & 0.71545 & -0.56344 \\
\hline Moda (EMV) & 0.97742 & 0.71207 & -0.53832 \\
\hline Mediana & 1.00013 & 1.00911 & 1.00431 \\
\hline$\sqrt{\widehat{\mathrm{R}}}$ - (crit. de conv.) & \\
\hline
\end{tabular}

A seguir, apresentamos os histogramas das amostras selecionadas para cada parâmetro e, nas figuras seguintes, os gráficos de convergência das duas cadeias com todos os pontos simulados pelo algoritmo.

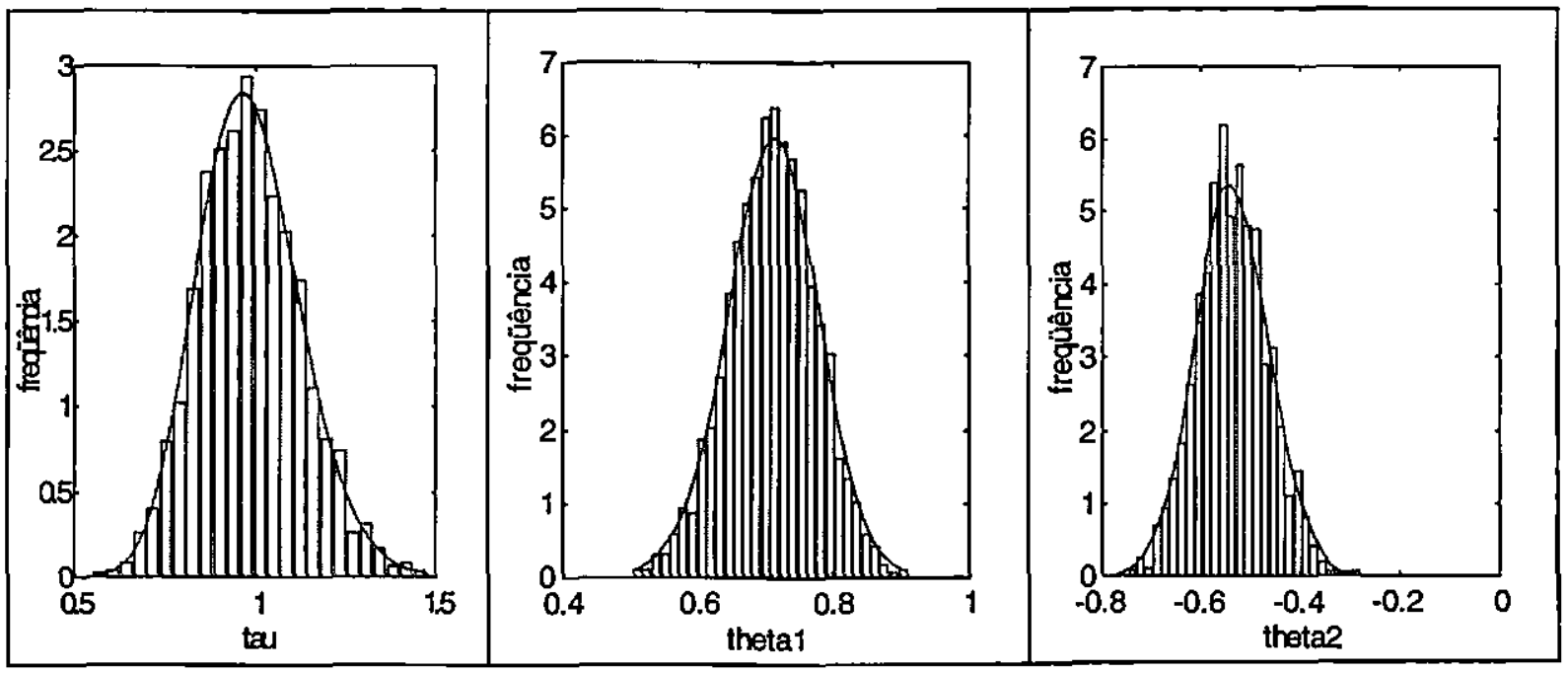

fig. 25 - Histogramas das amostras geradas por MCMC para $\tau, \theta_{1}$ e $\theta_{2}$. 

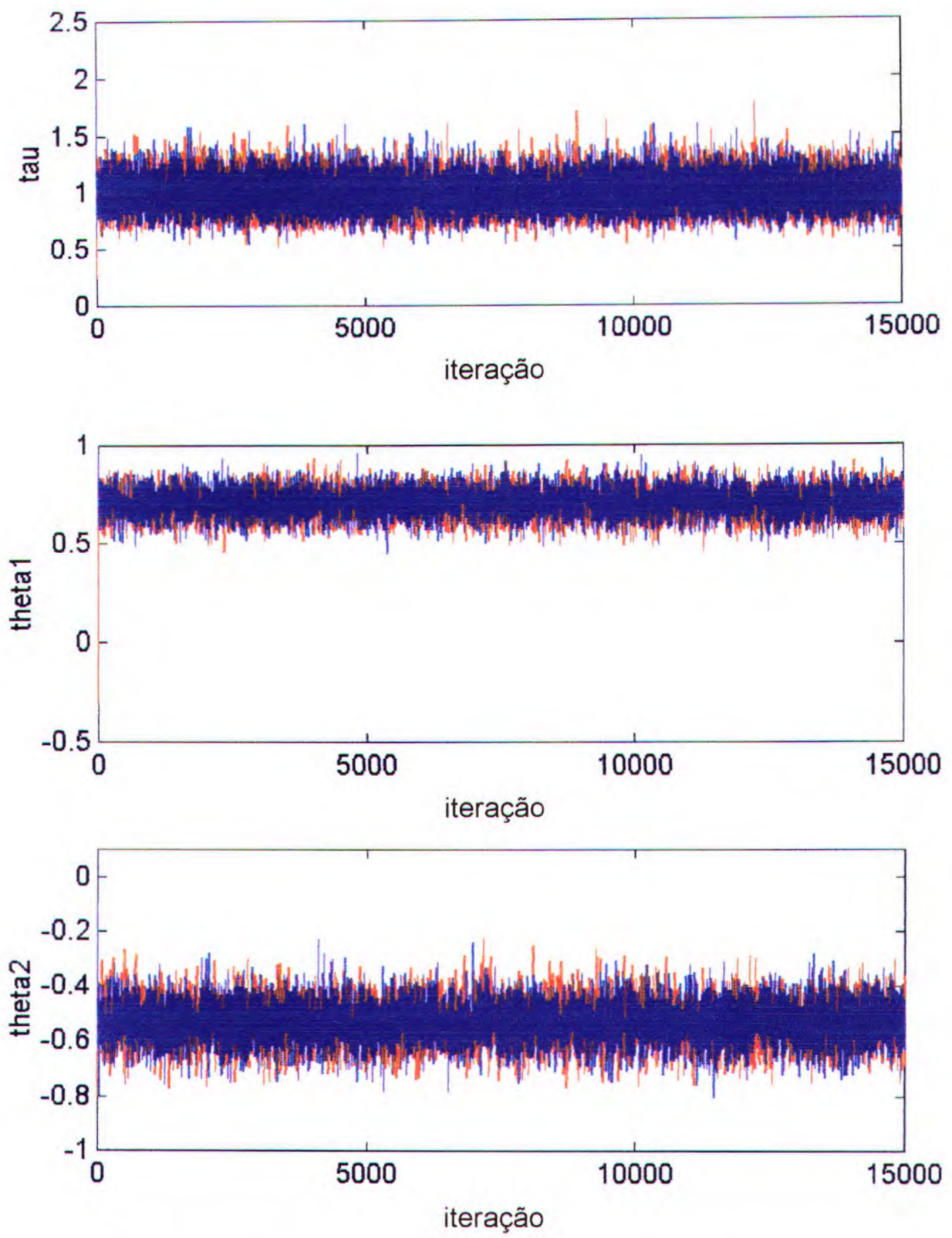

fig. 26 - Gráficos com todos os valores simulados para $\tau, \theta_{1}$ e $\theta_{2}$. 


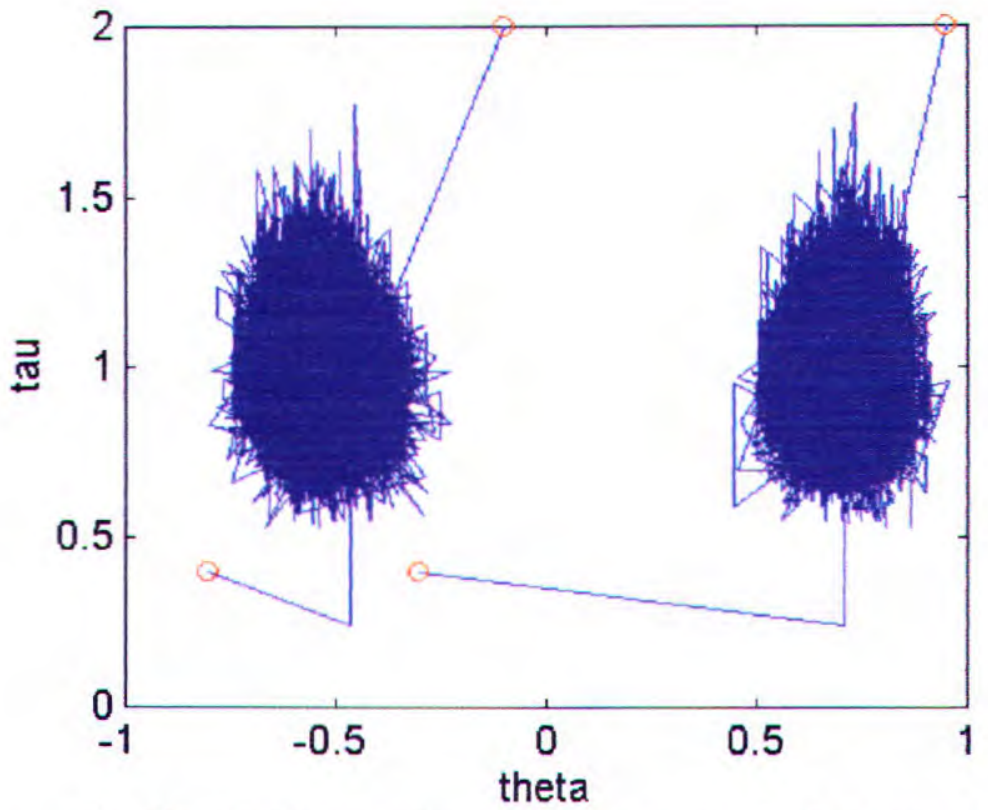

fig. 27 - Gráfico mostrando a convergência das duas cadeias geradas, com seus repectivos pontos de partida. 


\section{Capítulo 7 - Conclusão}

Neste projeto de dissertação, estudamos os modelos para séries temporais estacionárias do tipo $\mathrm{AR}(\mathrm{p})$ e $\mathrm{MA}(\mathrm{q})$ que são de muita utilidade em problemas de previsão de séries temporais. Mais especificamente, estudamos métodos de estimativas de máxima verossimilhança para estes modelos. O objetivo principal deste trabalho foi propor o uso de algoritmos de simulação de Monte Carlo com Cadeia de Markov (MCMC) como método de obtenção das estimativas de máxima verossimilhança.

As principais conclusões obtidas com o desenvolvimento deste trabalho foram:

- O uso de algoritmos MCMC para o cálculo das estimativas de máxima verossimilhança em modelos $\mathrm{AR}(\mathrm{p})$ mostra-se viável quando adota-se a função de verossimilhança exata, pois tais estimativas só podem ser calculadas fazendo-se simplificações desta função e usando-se métodos numéricos. Com o uso de algoritmos MCMC essas simplificações não são necessárias.

- O uso de algoritmos MCMC em modelos MA(q) para o cálculo das estimativas de máxima verossimilhança apresenta a vantagem de que além de estimar de forma precisa os parâmetros do modelo, não necessita o uso de "backforecasting" como no método estimação de máxima verossimilhança incondicional, o qual é um tanto arbitrário ao se assumir valores nulos para $\left[e_{t}\right], t=n+1, n+2, \ldots$. Usando MCMC este problema é contornado pois $a_{1-q}, a_{2-q}, \ldots, a_{0}$ são gerados da sua distribuição de probabilidade. Além disso, o uso de MCMC evita um algoritmo de maximização da função $S(\theta)$. 
- Na comparação do uso do algoritmo MCMC com os métodos numéricos, os resultados mostraram que as estimativas obtidas via MCMC estão muito próximas das obtidas com os outros métodos no caso dos modelos $\mathrm{AR}(\mathrm{p})$. No caso dos modelos $\mathrm{MA}(\mathrm{q})$ a diferença entre as estimativas para o parâmetro $\theta$ mostraram-se um pouco maiores, porém com resultados bastante satisfatórios. Uma desvantagem que surge no uso de MCMC em modelos MA(q) é o tempo computacional. Devido à necessidade de operaçőes com matrizes de ordem $n \times n$ (grande), estes algoritmos mostram-se mais lentos que os demais. No entanto, a vantagem dos métodos numéricos pode diminuir muito à medida que a discretização do intervalo de variação dos parâmetros nestes métodos aumenta. Esta discretização também afeta a precisão desses métodos.

Uma possível continuação do presente trabalho é a extensão da aplicação dos algoritmos MCMC em modelos mais gerais como os modelos Mistos Auto-regressivos - Médias Móveis (ARMA(p,q)), utilizando-se a expressão da função de verossimilhança exata destes modelos apresentada em [Box, Jenkins e Reinsel - 1994]. 


\section{Bibliografia}

Anderson, T.W.; Mentz, R.P.; Jarma, N.M.; Martinez, C.I. (1996). Simulations of Iterative Procedures for Maximum Likelihood Estimation in MA(1) Models, Commun. Statist. Simula., 25(4), 851-865.

Box, G.E.; Cox, D.R. (1964). An Analysis of Transformations, Journal of the Royal Statistic Society, B, Vol. 6, pp. 211-252.

Box, G.E.; Jenkins, G.M.; Reinsel, G.C. (1994). Time Series Analysis Forecasting and Control, 3th ed., Prentice Hall, New Jersey.

Casella, G.; George, E.I. (1992). Explaining the Gibbs Sampler, The American Statistician, Vol. 46, No. 3, pp. 167-174.

Chatfield, C. (1989). The Analysis of Time Series: An Introduction, 4th ed., Chapman and Hall, New York.

Chib, S.; Greenberg, E. (1995). Understanding the Metropolis-Hastings Algorithm, The American Statistician, Vol. 49, No. 4, pp. 327-335.

Gelman, A.; Rubin, D.B. (1992). Inference from Iterative Simulation Using Multiple Sequences, Statistical Science, Vol. 7, No. 4, pp. 457-511.

Geyer, C.J. (1994). On the Convergence of Monte Carlo Maximum Likelthood Calculations, Journal of Royal Statistical Society, 56, No.1, pp. 261-274. 
Geyer, C.J.; Thompson, E.A. (1992). Constrained Monte Carlo Maximum Likelihood for Dependent Data, Journal of Royal Statistical Society, 54, No. 3, pp. 657-699.

Miller, J.W. (1995). Exact Maximum Likelihood Estimation in Autoregressive Process, Journal of Time Series Analysis, Vol. 16, No. 6, pp. 607-615.

Morettin, P.A.; Toloi, C.M.C. (1981). Modelos para Previsão de Séries Temporais, IMPA, Rio de Janeiro. 


\section{Apêndice}

\section{Previsão}

Um dos objetivos mais importantes dentro da análise de séries temporais é a previsão dos valores futuros de uma série observada. Suponhamos que conhecemos as observações $\mathbf{z}_{t}^{\prime}=\left(\mathrm{z}_{1}, \mathrm{z}_{2}, \ldots, \mathrm{z}_{t}\right)$ e desejamos prever o valor esperado de $\mathrm{z}_{t+l}, l \geq 1$. Suponhamos também que $z_{t+l}$ seja uma combinação linear de $z_{t}, z_{t-1}, z_{t-2}, \ldots$, e que portanto pode ser escrito também como combinação linear dos ruídos $a_{t}, a_{t-1}, a_{t-2}, \ldots$.

Agora, seja $\hat{z}_{t}(l)$ a previsão de $\mathrm{z}_{t+l}$. Suponhamos que a melhor previsão seja dada por:

$$
\hat{\mathbf{z}}_{t}(l)=\psi_{i}^{*} a_{t}+\psi_{t+1}^{*} a_{t-1}+\psi_{t+2}^{*} a_{t-2}+\ldots
$$

Então, usando o fato que

$$
\mathrm{z}_{t+l}=a_{\mathrm{t}+t}+\psi_{1} a_{\mathrm{t}+l-1}+\psi_{2} a_{t+i-2}+\ldots
$$

o erro quadrático médio da previsão é

$$
E\left[\mathrm{z}_{t+l}-\hat{\mathbf{z}}_{t}(l)\right]^{2}=\left(1+\psi_{1}^{2}+\ldots+\psi_{l-1}^{2}\right) \sigma_{a}^{2}+\sum_{j=0}^{\infty}\left(\psi_{l+j}-\psi_{i+j}^{*}\right)^{2} \sigma_{a}^{2}
$$

Pode-se mostrar que este valor é minimizado quando $\psi_{i+j}^{*}=\psi_{i+j}$. Portanto temos

$$
\begin{aligned}
\mathrm{z}_{t+l} & =\left(a_{t+l}+\psi_{1} a_{t+l-1}+\ldots+\psi_{l-1} a_{t+1}\right)+\left(\psi_{l} a_{t}+\psi_{t+1} a_{t-1}+\ldots\right) \\
& =e_{t}(l)+\hat{\mathbf{z}}_{t}(l)
\end{aligned}
$$

onde $e_{t}(l)$ é o erro da previsão $\hat{\mathrm{z}}_{t}(l)$. 
Usando a expressão (A1) e o fato que $E\left[a_{t+j} \mid \mathbf{z}_{t}\right]=0$ para $j>0$, concluímos que

$$
\hat{\mathrm{z}}_{\mathrm{t}}(l)=\psi_{t} a_{\mathrm{t}}+\psi_{t+1} a_{t-1}+\ldots=E\left[\mathrm{z}_{t+1} \mid \mathbf{z}_{t}\right]
$$

Desse resultado, fazendo $l=1$ podemos calcular previsões de um passo à frente para um modelo $A R(p)$ dado por

$$
\mathrm{z}_{t+l}=\phi_{1} \mathrm{z}_{t+1-1}+\phi_{2} \mathrm{z}_{t+1-2}+\ldots+\phi_{\mathrm{p}} \mathrm{z}_{t+\mathrm{l}-\mathrm{p}}+a_{t+l}
$$

Denotando por $\hat{z}_{t}(1)$ a previsão de $z_{t+1}$, de (A2) temos que:

$$
\begin{aligned}
\hat{\mathbf{z}}_{t}(1) & =E\left[\mathbf{z}_{t+1} \mid \mathbf{z}_{t}\right] \\
& =\phi_{1} E\left[\mathbf{z}_{t} \mid \mathbf{z}_{t}\right]+\phi_{2} E\left[\mathbf{z}_{t-1} \mid \mathbf{z}_{t}\right]+\ldots+\phi_{p} E\left[\mathbf{z}_{t+1-p} \mid \mathbf{z}_{t}\right]+E\left[a_{t+1} \mid \mathbf{z}_{t}\right]
\end{aligned}
$$

Como $E\left[\mathbf{z}_{t-j} \mid \mathbf{z}_{t}\right]=z_{t}$ para $j \geq 0$ e $E\left[a_{t+1} \mid \mathbf{z}_{t}\right]=0$, segue que

$$
\hat{z}_{t}(1)=\phi_{1} z_{t}+\phi_{2} z_{t-1}+\ldots+\phi_{p} z_{t+1-p}
$$

Para $l>1$, podemos calcular as previsões por:

$$
\hat{\mathrm{z}}_{l}(l)=\phi_{1}\left[\mathrm{z}_{t+i-1}\right]+\phi_{2}\left[\mathrm{z}_{t+l-2}\right]+\ldots+\phi_{p}\left[\mathrm{z}_{t+l-p}\right]
$$

onde

$$
\begin{aligned}
& {\left[\mathrm{z}_{t+\mathrm{k}}\right]=\hat{\mathrm{z}}_{t}(k) ; \quad k>0} \\
& {\left[\mathrm{z}_{t+\mathrm{k}}\right]=\mathrm{z}_{t+\mathrm{k}} ; \quad k \leq 0}
\end{aligned}
$$

Para previsão com modelos MA, a equação (A2) pode ser usada diretamente, fazendo

$$
\begin{aligned}
& {\left[a_{t+\mathrm{k}}\right]=0 ; \quad k>0} \\
& {\left[a_{t+\mathrm{k}}\right]=a_{\mathrm{t}+\mathrm{k}} ; \quad k \leq 0}
\end{aligned}
$$

